\title{
Differential operators on locally compact groups
}

\author{
By
}

\author{
Takashi EDAMATSU
}

\section{Introduction}

The differential operators on a $C^{\infty}$-manifold are closely related to the distribution theory on it and can be defined simply by local character (e.g. [7]). $F$. Bruhat [5] showed that a notion of differential operators can be introduced also on any locally compact group $G$ on the basis of his distribution theory. The differential operators in Bruhat's sense act on his space $\mathscr{D}(G)$ of compactly supported regular functions, and take a quite natural form consistent with those on Lie groups ([5], p. 66). But his very method of defining them seems somewhat intricate and unrefined. In the present paper we intend to get the "differential operators" on $G$ rather by local character. Here, as the base space on which they act, we take the $C^{\infty}$-class $\mathcal{E}_{\infty}(G)$ (see below) on $G$ rather than $\mathscr{D}(G)$ or $\mathcal{E}(G)$, the Bruhat space of all regular functions.

The substance of the $n$-times derivable distributions on $G$ in Bruhat's sense $(n=\infty, 1,2, \cdots)([5]$, p. 67$)$ has been left unknown in case $G$ is not locally connected. On the other hand, we have the $C^{n}$-classes on $G$, denoted by $\mathcal{E}_{n}(G)$ $(n=\infty, 1,2, \cdots)$ and defined by using one-parameter subgroups of $G$, as natural generalizations of those on Lie groups. They were first introduced in J. Riss [12] for the abelian case and lately generalized to any $G$ in H. Boseck, G. Czichowski and K. P. Rudolph [3]. ${ }^{(1)} \mathcal{E}(G)$ is included in $\mathcal{E}_{\infty}(G)$. Let $\mathscr{D}_{n}(G)$ be the linear subspace of $\mathcal{E}_{n}(G)$ consisting of the compactly supported functions. We equip each $\mathscr{D}_{n}(G)$ (resp. $\mathcal{E}_{n}(G)$ ) with an inductive limit (resp. projective) topology analogous to that of $\mathscr{D}(G)$ (resp. $\mathcal{E}(G)$ ). The differential operators on $G$ in our sense are defined as the support-decreasing continuous linear maps on $\mathcal{E}_{\infty}(G)$ or, what is substantially the same (see section 2,1 ), on $\mathscr{D}_{\infty}(G)$. The purpose of the present paper is twofold. One aim is to study the differential operators thus defined in comparison with Bruhat's ones. And the other is to show that for each $n$, the $n$-times derivable distributions in Bruhat's sense just coincide with the functions in $\mathcal{E}_{n}(G)$ no matter $G$ is locally connected or not.

The paper consists of three chapters. Chapter 0 arranges some classical facts concerning the general Lie theory on locally compact groups ([9]) and their dimension. The contents of Chapters 1 and 2 are as follows.

The arguments on $\mathscr{D}_{n}(G)$ and $\mathcal{E}_{n}(G)$ in [3] are not necessarily enough for

(1) In the notation of [3], $\mathcal{E}_{n}(G)$ is designated as $C_{w}^{n}(G ; C)(n=\infty, 1,2, \cdots)$. Communicated by Prof. Yoshizawa, February 26, 1987 
us. For instance, the topological consideration on $\mathscr{D}_{n}(G)$ was made there within the $(L F)$-space theory by assuming the first countability of $G$. So we had better reconstruct the theory of these spaces in a form appropriate for our purpose and so as to apply to any $G$. This somewhat tiresome work is performed in Charpter 1 (sections 1.1-1.5).

Let $\mathscr{D}^{\prime}(G), \mathcal{E}^{\prime}(G), \mathscr{D}_{\infty}^{\prime}(G)$ and $\mathcal{E}_{\infty}^{\prime}(G)$ be the topological dual of the spaces concerned. They are equipped with the strong topologies of dual. $\mathscr{D}^{\prime}(G)$ $\left(\supseteq \mathcal{E}^{\prime}(G)\right.$ ) is nothing but the space of Bruhat's distributions. If $G$ is abelian, $\mathscr{D}_{\infty}^{\prime}(G)$ is the space of Riss' distributions ([12]). In $\mathbf{1 . 5}$ the relations among these four spaces are also clarified. Especially, we shall have $\mathcal{E}_{\infty}^{\prime}(G) \subseteq \mathcal{E}^{\prime}(G)$ as set, and $\mathcal{E}_{\infty}^{\prime}(G) \subsetneq \mathcal{E}^{\prime}(G)$ if $G$ is not locally connnected. In 1.6, the last section of Chapter 1 , it is shown that $\mathcal{E}_{\infty}^{\prime}(G)$ (resp. $\mathcal{E}^{\prime}(G)$ ) is a topological algebra under the convolution and (algebraically) isomorphic to the algebra of all left invariant continuous linear maps on $\mathcal{E}_{\infty}(G)$ (resp. $\mathcal{E}(G)$ ).

Chapter 2 is concerned with the differential operators. Section 2.1 is devoted to the definition. It is worth noting that our definition by local character reflects the property of the continuous derivations on $\mathcal{E}_{\infty}(G)$ and $\mathscr{D}_{\infty}(G)$ (see Lemmas 2.1 and 2.3). The basic elements in our treatment are, in view of its nature, the derivations associated to one-parameter subgroups of $G$. Sections 2.2 and 2.3 are devoted to a study of them. The totality of our differential operators on $G$ is denoted by $\overline{\boldsymbol{D}}(G) . \quad \overline{\boldsymbol{D}}(G)$ is endowed with a reasonable topology (Lemma 2.7 and Definition 2.6) which makes $\bar{D}(G)$ a topological algebra.

The main results of the paper are Theorems 2.3 and 2.4. Theorem 2.3 gives to the elements of $\overline{\boldsymbol{D}}(G)$ an explicit expression, similar to that of Bruhat's differential operators, in terms of the functions in $\mathcal{E}_{\infty}(G)$ and the derivations associated to one-parameter subgroups. This theorem is obtained essentially by taking after the arguments in [5], $\mathrm{n}^{\circ} 12(2.4,2.5)$. From the observation of the process to reach this theorem we can see that the differential operators in Bruhat's sense are nothing but the support-decreasing continuous linear maps on $\mathscr{D}(G)$ (or, what is the same, on $\mathcal{E}(G)$ ) and that they form substantially a subset of $\overline{\boldsymbol{D}}(G)(\mathbf{2 . 7})$. We can determine, among all elements of $\overline{\boldsymbol{D}}(G)$, the derivations and the left invariant elements (2.6).

Theorem 2.4 is not only significant by itself but also necessary for later use. Let $\mathcal{E}_{\infty}^{\prime}(G ; e)$ (resp. $\mathcal{E}^{\prime}(G ; e)$ ) be the topological subalgebra of $\mathcal{E}_{\infty}^{\prime}(G)$ (resp. $\mathcal{E}^{\prime}(G)$ ) of the elements with support in $\{e\}, e$ being the idantity of $G$, and $\overline{\boldsymbol{D}}_{l}(G)$ the topological subalgebra of $\bar{D}(G)$ of the left invariant elements. Theorem 2.4 asserts that $\mathcal{E}_{\infty}^{\prime}(G ; e)$ and $\mathcal{E}^{\prime}(G ; e)$ coincide with each other as topological algebra (in contrast to the fact that $\mathcal{E}_{\infty}^{\prime}(G) \subsetneq \mathcal{E}^{\prime}(G), G$ being locally nonconnected) and that they are topologically isomorphic to $\overline{\boldsymbol{D}}_{l}(G)$. These facts are needed in order to combine our discussions with ones in [5], and used in 2.8 and 2.10. In 2.8 we describe the center of $\overline{\boldsymbol{D}}_{l}(G)$ by means of the "adjoint representation" of $G$, as in the case of Lie groups. In 2.10. the final section, it is proved that the $n$-times derivable distributions in Bruhat's sense are no other than the functions in $\mathcal{E}_{n}(G)(n=\infty, 1,2, \cdots)$. To do so, the notion of the 
order of differential operators is also needed. We discuss it in $\mathbf{2 . 9}$.

In [6], where $G$ is compact, a class $\boldsymbol{D}(G)$ of differential operators was considered. The relation between $\boldsymbol{D}(G)$ and $\overline{\boldsymbol{D}}(G)$ is explained in 2.9. The contents of the sections 1.1-1.5, 2.2, 2.3 and 2.8 generalize all results in [6] to any locally compact $G$.

Notation. $G$ denotes a locally compact Hausdorff group ( $L C$ group) with identity $e$ and a fixed left Haar measure $d_{G}$. $\boldsymbol{R}$ (resp. $\boldsymbol{C}$ ) denotes the field of real (resp. complex) numbers with the usual topology. For a $\boldsymbol{C}$-valued function $f$ on $G$ and $x \in G$, the functions ${ }_{x} f, f_{x}, \check{f}$ and $\bar{f}$ are defined by ${ }_{x} f(y)=f(x y)$, $f_{x}(y)=f(y x), \check{f}(y)=f\left(y^{-1}\right)$ and $\bar{f}(y)=\overline{f(y)}$ (complex conjugate) $(y \in G) . \quad \Delta_{G}$ denotes the modular function on $G$ such that $\Delta_{G} d_{G}$ is a right Haar measure. $R(G)$ denotes the totailty of one-parameter subgroups of $G$, where a one-parameter subgroup means a continuous homomorphism of the additive group $\boldsymbol{R}$ into $G$. $c(G)$ denotes the connected component of $e$ in $G$. If $N, N^{\prime}$ are closed normal subgroups of $G$ s.t. $N \cong N^{\prime}$, then $\pi_{N}^{G}$ denotes the canonical homomorphism of $G$ onto $G / N$, and $\pi_{N^{\prime} N}^{G}$ that of $G / N$ onto $G / N^{\prime}$. For a closed subset $\Gamma$ of $G$, Com $(\Gamma)$ denotes the totality of compact subsets of $\Gamma$. For a set $\mathcal{K}$ of $\boldsymbol{C}$-valued functions on $G, \mathcal{K}^{+}$denotes the subset of $\mathcal{K}$ consisting of the $\boldsymbol{R}$-valued nonnegative functions.

\section{Chapter 0. Preliminaries}

\subsection{The Lie algebra of $G$.}

Definition 0.1. $\boldsymbol{H}_{0}(G)$ denotes the totality of compact normal subgroups $N$ of $G$ such that $G / N$ is a Lie group. Here every discrete group is counted as Lie group. $G$ is called Lie-projective or simply pro-Lie if $\cap\left\{N \in \boldsymbol{H}_{0}(G)\right\}=\{e\}$.

$\boldsymbol{H}_{0}(G)$ is closed under the formation of finite intersections of the members (e.g. [5], $\mathrm{n}^{\circ} 1$ ). So it is lower directed under inclusion. Throughout the paper we consider $\boldsymbol{H}_{0}(G)$ as a directed set in this sense. Note that the Lie-projectivity of $G$ is equivalent to that for each neighbourhood $V$ of $e$, there exists an $N \in$ $\boldsymbol{H}_{0}(G)$ s.t. $N \leqq V$. For any $N, N^{\prime} \in \boldsymbol{H}_{0}(G)$ s. t. $N \cong N^{\prime}$, let $\pi_{N^{\prime} N}^{G}$ denote the canonical homomorphism of $G / N$ onto $G / N^{\prime}$. Then an inverse system $\left\{G / N, \pi_{N^{\prime} N}^{G}\right\}$ of Lie groups is obtained. A Lie-projective $G$ is isomorphic to the limit of this system: $G \cong \lim _{\lfloor}\left\{G / N, \pi_{N^{\prime} N}^{G}\right\}$. The isomorphy is given by the map

$$
\iota_{G}: x \in G \longmapsto\left(\pi_{N}^{G}(x)\right)_{N \in H_{0}(G)},
$$

where $\pi_{N}^{G}$ denotes the canonical homomorphism of $G$ onto $G / N$. Not all $L C$ groups are Lie-projective. But, by the well known approximation theorem, $G$ is Lie-projective if it is almost connected (i. e., $G / c(G)$ is compact, $c(G)$ denoting the identity component of $G)([10]$, p. 175). Besides, any $G$ contains an almost connected (hence Lie-projective) open subgroup (loc. cit. p. 54).

We define the Lie algebra of $G$ following R.K. Rashof [9]. First suppose 
$G$ is Lie-projective: $G \cong \lim _{\{}\left\{G / N, \pi_{N^{\prime} N}^{G}\right\}\left(N, N^{\prime} \in \boldsymbol{H}_{0}(G), N \cong N^{\prime}\right)$. Then an inverse system $\left\{L(G / N), d \pi_{N^{\prime} N}^{G}\right\}$ of finite-dimensional locally convex real Lie algebras is obtained, where $L(G / N)$ is the Lie algebra of $G / N$, a Lie group, and $d \pi_{N^{\prime} N}^{G}$ the differential of $\pi_{N^{\prime} N}^{G}$. Thus, as its limit, we have a locally convex real Lie algebra $g(G)$ (infinite-dimensional in general): $g(G)=\varliminf_{\lfloor}\left\{L(G / N), d \pi_{N^{\prime} N}^{G}\right\}$. The following lemma is proved by the sujectivity of every $d \pi_{N^{\prime} N}^{G}$ and the theory of linearly compact vector spaces.

Lemma 0.1 ([9], Theorem 2.3). Let $G$ be Lie-projective. The canonical projection of $\mathrm{g}(G)$ into each component $L(G / N)\left(N \in \boldsymbol{H}_{0}(G)\right)$ is surjective.

Now let $G$ be arbitrary. Since $c(G)$ is Lie-projective, $g(c(G))$ can be defined: $g(c(G))=\varliminf\left\{L(c(G) / K), d \pi_{K^{\prime} K}^{c(G)}\right\} \quad\left(K, K^{\prime} \in \boldsymbol{H}_{0}(c(G)), K \subseteq K^{\prime}\right)$.

Definition 0.2. Any locally convex real Lie algebra isomorphic to $g(c(G))$ is called the Lie algebra of $G$ and denoted symbolically by $L(G)$.

This definition generalizes the notion of Lie algebra of Lie groups to any $L C$ groups. Let us note that $G$ and $c(G)$ have the same Lie algebra.

Remark 0.1. If $G$ is Lie-projective, then $g(G) \cong g(c(G))$. Hence $g(G)$, as well as $g(c(G))$, gives a realization of $L(G)$.

Before seeing this, we set two simple lemmas.

Lemma 0.2 ([10], p. 192). Let $N$ be a compact normal subgroup of $G$. Then $\pi_{N}^{G}(c(G))=c(G / N)$.

Lemma 0.3. Let $\boldsymbol{H}$ be a lower directed family of compact subgroups of $G$ s.t. $\cap\{K \in \boldsymbol{H}\}=\{e\}$ (i.e., each neighbourhood of e contains some $K \in \boldsymbol{H}$ ). Then, for each $N \in \boldsymbol{H}_{0}(G)$, there exists a $K \in \boldsymbol{H}$ s.t. $K \cong N$.

Proof. Obvious since $G / N$, a Lie group, has no small subgroups. q.e.d.

Proof of Remark 0.1. For $N \in \boldsymbol{H}_{0}(G)$, put $\tilde{N}=N \cap c(G)$. Then $\tilde{N} \in \boldsymbol{H}_{0}(c(G))$ since $c(G) / \tilde{N} \cong c(G) N / N$ (a Lie group as a closed subgroup of $G / N$ ). Put $\boldsymbol{H}=$ $\left\{\tilde{N} ; N \in \boldsymbol{H}_{0}(G)\right\}$. Then, as well as $\boldsymbol{H}_{0}(G), \boldsymbol{H}$ is lower directed and satisfies $\bigcap\{K \in \boldsymbol{H}\}=\{e\}$. Hence, by Lemma 0.3 , it is cofinal in $\boldsymbol{H}_{0}(c(G))$. Therefore, for our proof, it suffices to show that $\mathrm{g}(G)$ is isomorphic to the locally convex Lie algebra $\lim _{\lfloor}\left\{L(c(G) / \tilde{N}), d \pi_{\tilde{N}^{\prime} \tilde{N}}^{c(G)}\right\}\left(N, N^{\prime} \boldsymbol{H}_{0}(G), N \leqq N^{\prime}\right)$, which we denote by $\mathrm{g}^{\prime}\left(c(G)\right.$ ). Now, for each $N \in \boldsymbol{H}_{0}(G)$, let $f_{. N}$ denote the canonical isomorphism of $c(G) / \tilde{N}$ onto $c(G) N / N$. Then we have the following commutative diagram: 


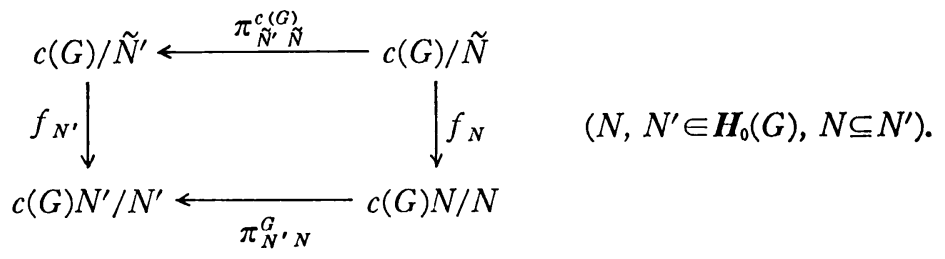

Here $c(G) N / N$ (resp. $c(G) N^{\prime} / N^{\prime}$ ) is the identity commponent of $G / N$ (resp. $G / N^{\prime}$ ) (Lemma 0.2). Therefore this diagram yields the following commutative one:

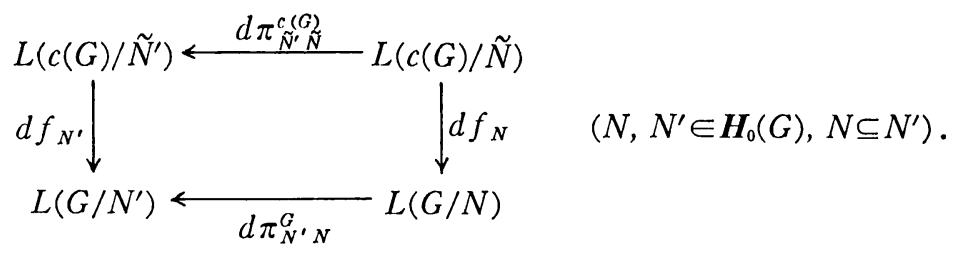

Since $d f_{N}$ for each $N \in \boldsymbol{H}_{0}(G)$ is an isomorphism of $L(c(G) / \tilde{N})$ onto $L(G / N)$, this diagram shows that $\mathrm{g}^{\prime}(c(G))$ is isomorphic to $\mathrm{g}(G)$ under the map

$$
\left(Y_{\tilde{N}}\right)_{N \in H_{0}(G)} \in \mathrm{g}^{\prime}(c(G)) \longmapsto\left(\left(d f_{N}\right) Y_{\dot{N}}\right)_{N \in H_{0}(G)} \in \mathrm{g}(G),
$$

completing the proof. q.e.d.

From the definition we see that the locally convex linear space $L(G)$ is minimal, i.e., isomorphic to $\boldsymbol{R}^{I}$ for some power $I$. Therefore, in particular, it is a Baire space and also barrelled. Let us note that $L(G)$ has a topological linear base $\left\{X^{(i)}\right\}_{i \in I}$ (i. e., the map $\left(c_{i}\right)_{i \in I} \in \boldsymbol{R}^{I \mapsto} \mapsto \sum_{i \in I} c_{i} X^{(i)}$ (unordered sum) is a topological linear isomorphism of $\boldsymbol{R}^{I}$ onto $L(G)$ ).

0.2. Exponential map and one-parameter subgroups. First suppose $G$ is Lie-projective. For $X=\left(X_{N}\right)_{N \in H_{0}(G)} \in \mathfrak{g}(G)$ with $L(G / N)$-component $X_{N}$ we have

$$
\begin{aligned}
\pi_{N^{\prime} N}^{G}\left(\exp _{G / N}\right) & =\exp _{G / N^{\prime}}\left(d \pi_{N^{\prime} N}^{G}\right) X_{N} \\
& =\exp _{G / N^{\prime}} X_{N^{\prime}} \quad\left(N, N^{\prime} \in \boldsymbol{H}_{0}(G), N \cong N^{\prime}\right),
\end{aligned}
$$

where each $\exp _{G / N}$ denotes the usual exponential map of $L(G / N)$ into $G / N$, a Lie group. Hence $\left(\exp _{G / N} X_{N}\right)_{N \in H_{0}(G)} \in \ell_{G}(G)$. Thus we can define a map of $\mathrm{g}(G)$ into $G$ by

$$
X \longmapsto \iota_{G}^{-1}\left(\left(\exp _{G / N} X_{N}\right)_{N \in H_{0}(G)}\right) .
$$

Now let $G$ be arbitrary. Then a map of $g(c(G))$ into $G$ is defined as

$$
Y \longmapsto c_{c(G)}^{-1}\left(\left(\exp _{c(G) / K} Y_{K}\right)_{\left.K \in H_{0}(c(G))\right)},\right.
$$

where $Y=\left(Y_{K}\right)_{K \in H_{0}(c(G))} \in \mathrm{g}(c(G))$.

Definition 0.3. For any $L C$ group $G, \exp _{G}$ denotes the map of $L(G)$ into 
$G$ which is given by $(0.3)$ if $L(G)$ is realized by $g(c(G))$.

Since each $\exp _{c(G) / K}\left(K \in \boldsymbol{H}_{0}(c(G))\right)$ is continuous, it is plain that $\exp _{G}$ is continuous. Note that the maps $\exp _{G}$ and $\exp _{c(G)}$ are the same. In particular, $\exp _{G}$ maps $L(G)$ into $c(G)$.

Remark 0.2. Let $G$ be Lie-projective. If $L(G)$ is realized by $g(G), \exp _{G}$ is given by $(0.2)$.

Proof. Define the map exp' (resp. exp") of $\mathrm{g}(G)$ (resp. $\mathrm{g}(c(G))$ ) into $G$ by (0.2) (resp. (0.3)). Suppose that $X=\left(X_{N}\right)_{N \in H_{0}(G)} \in \mathrm{g}(G)$ corresponds to $Y=$ $\left(Y_{K}\right)_{K \in H_{0}(c(G))} \in \mathrm{g}(c(G))$ under the isomorphism of $\mathrm{g}(c(G))$ onto $\mathrm{g}(G)$ established in the proof of Remark 0.1. Then, for our proof, it suffices to show that $\exp ^{\prime} X=\exp ^{\prime \prime} Y$ holds. For each $N \in \boldsymbol{H}_{0}(G)$, let $P_{N}^{G}$ denote the canonical projection of $\iota_{G}(G)$ onto $G / N$. Then $\pi_{N}^{G}=P_{N}^{G}{ }^{\circ} \iota_{G}$. Hence

$$
\pi_{N}^{G}\left(\exp ^{\prime} X\right)=\exp _{G / N} X_{N} .
$$

Similarly we have for $K \in \boldsymbol{H}_{0}(c(G))$,

$$
\pi_{K}^{c(G)}\left(\exp ^{\prime \prime} Y\right)=\exp _{c(G) / K} Y_{K} .
$$

Now retain the notations as in the proof of Remark 0.1. Then, by assumption, $X_{N}=\left(d f_{N}\right) Y_{\tilde{N}}\left(N \in \boldsymbol{H}_{0}(G)\right)$. Also, it is evident that $f_{N} \circ \pi_{\tilde{N}}^{c(G)}=\pi_{N}^{G}\left(N \in \boldsymbol{H}_{0}(G)\right)$. By these together with $\left(^{*}\right)$ and $(* *)$ we have

$$
\begin{aligned}
& \pi_{N}^{G}\left(\exp ^{\prime} X\right)=\exp _{G / N} X_{N}=\exp _{G / N}\left(d f_{N}\right) Y_{\tilde{N}} \\
= & f_{N}\left(\exp _{c(G) / \tilde{N}} Y_{\tilde{N}}\right)=f_{N}\left(\pi_{\tilde{N}}^{c(G)}\left(\exp ^{\prime \prime} Y\right)\right)=\pi_{N}^{G}\left(\exp ^{\prime \prime} Y\right) .
\end{aligned}
$$

Since $N\left(\in \boldsymbol{H}_{0}(G)\right)$ is arbitrary, this shows that $\iota_{G}\left(\exp ^{\prime} X\right)=\iota_{G}\left(\exp ^{\prime \prime} Y\right)$, i. e., that $\exp ^{\prime} X=\exp ^{\prime \prime} Y$. q. e. d.

The above $\left(^{*}\right)$ is used later on. So we record it formally: Let $G$ be Lieprojective. Then, for $X=\left(X_{N}\right)_{N \in H_{0}(G)} \in g(G)$,

$$
\pi_{N}^{G}\left(\exp _{G} X\right)=\exp _{G / N} X_{N},
$$

Lemma 0.4. The map $X \in L(G) \mapsto \exp _{G} t X$ ( $t$ real parameter) is a bijection of $L(G)$ onto $R(G)$, the set of all one-parameter subgroups of $G$.

Proof. Since $\exp _{G}=\exp _{c(G)}$ and obviously $R(G)=R(c(G))$, we can assume that $G$ is connected. For $X=\left(X_{N}\right)_{N \in H_{0}(G)} \in g(G)$, put $\alpha_{X}(t)=\left(\exp _{G / N} t X_{N}\right)_{N \in H_{0}(G)}$ $(t \in R)$. Then the map $X \mapsto \alpha_{X}$ gives a bijection of $\mathfrak{g}(G)$ onto $R\left(\iota_{G}(G)\right)$. Hence the lemma. q.e.d.

On the basis of this lemma we make the following convention which is kept throughout the paper.

Convention. $R(G)$ is regarded as a locally convex real Lie algebra isomor- 
phic to $L(G)$ under the bijection $X \mapsto \exp _{G} t X$ of $L(G)$ onto $R(G)$.

Thus, if $\alpha, \beta \in R(G)$ are given as $\alpha(t)=\exp _{G} t X, \beta(t)=\exp _{G} t Y(X, Y \in L(G)$, $t \in \boldsymbol{R})$, then $(a \alpha+b \beta)(t)=\exp _{G} t(a X+b Y)(a, b \in \boldsymbol{R})$ and $[\alpha, \beta](t)=\exp _{G} t[X, Y]$. The locally convex linear space $R(G)$ is minimal and so Baire and barrelled. It is plain that the map $(\alpha, t) \in R(G) \times \boldsymbol{R} \mapsto \alpha(t) \in G$ is continuous.

Using Lemma 0.1 and (0.4), we have the following

Lemma 0.5. ([9], Theorem 3.5). The set $\left\{\exp _{G} X ; X \in L(G)\right\}$ generates a dense subgroup of $c(G)$.

0.3. Differential of group homomorphisms. Let $\theta$ be a continuous homomorphism of $G$ into another $L C$ group $G^{\prime}$. Then, for each $X \in L(G)$, the map $t \in \boldsymbol{R} \mapsto \theta\left(\exp _{G} t X\right)$ belongs to $R\left(G^{\prime}\right)$. Therefore, by Lemma 0.4 , one has a unique element $X^{\prime} \in L(G)$ s.t.

$$
\theta\left(\exp _{G} t X\right)=\exp _{G^{\prime}} t X^{\prime} \quad(t \in \boldsymbol{R}) .
$$

Definition 0.4. Let $G, G^{\prime}$ and $\theta$ be as above. The map carrying each $X \in$ $L(G)$ to $X^{\prime} \in L\left(G^{\prime}\right)$ determined by $(0.5)$ is called the differential of $\theta$ and denoted by $d \theta$ or $\bar{\theta}$.

From $\theta\left(\exp _{G} t X\right)=\exp _{G^{\prime}} t \bar{\theta}(X)(t \in \boldsymbol{R}, X \in L(G))$, one has

$$
\operatorname{ker} \bar{\theta}=\left\{X \in L(G) ; \exp _{G} t X \in \operatorname{ker} \theta \text { for all } t \in \boldsymbol{R}\right\} \text {. }
$$

For a composition $\eta \circ \theta$ of group homomorphisms, $(\eta \circ \theta)^{-}=\bar{\eta} \circ \bar{\theta}$ holds. Let us note that, in case $G$ is Lie-projective, $\bar{\pi}_{N}^{G}$ for each $N \in \boldsymbol{H}_{0}(G)$ behaves as the canonical projection of $\mathrm{g}(G)$ onto the component $L(G / N)$ (cf. Lemma 0.1). In fact, by $(0.4), \pi_{N}^{G}\left(\exp _{G} t X\right)=\exp _{G / N} t X_{N}(t \in R)$ for $X=\left(X_{N}\right)_{N \in H_{0}(G)} \in g(G)$, hence $\bar{\pi}_{N}^{G}(X)=X_{N}$.

Lemma 0.6. ${ }^{(2)}$ Let $G, G^{\prime}$ and $\theta$ be as above.

(i) $\bar{\theta}$ is a continous Lie algebra homomorphism of $L(G)$ into $L\left(G^{\prime}\right)$. The image $\bar{\theta}(L(G))$ is closed in $L\left(G^{\prime}\right)$ and $\bar{\theta}$ is open as a map to $\bar{\theta}(L(G))$.

(ii) If $\operatorname{ker}\left(\left.\theta\right|_{c(G)}\right)=\{e\},\left.\theta\right|_{c(G)}$ denoting the restriction of $\theta$ to $c(G)$, then $\operatorname{ker} \bar{\theta}=\{0\}$. If $\theta(c(G))=c\left(G^{\prime}\right)$, then $\bar{\theta}(L(G))=L\left(G^{\prime}\right)$.

Proof. Evidently we can assume that $G$ and $G^{\prime}$ are connected. Let us realize $L(G), L\left(G^{\prime}\right)$ by $\mathrm{g}(G), \mathrm{g}\left(G^{\prime}\right)$ respectively.

(i): Let $N^{\prime} \in \boldsymbol{H}_{0}\left(G^{\prime}\right)$. Since the Lie group $G^{\prime} / N^{\prime}$ has no small subgroups, we can choose $N \in \boldsymbol{H}_{0}(G)$ s.t. $N \leqq \operatorname{ker}\left(\pi_{N^{\prime}}^{G^{\prime}} \circ \theta\right)$. Then $\pi_{N^{\prime}}^{G^{\prime} \circ \theta}$ induces a continuous

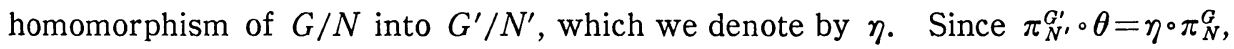
one has $\bar{\pi}_{N}^{G \prime} \circ \bar{\theta}=\bar{\eta}^{\circ} \bar{\pi}_{N}^{G}$. Here $\bar{\pi}_{N}^{G}$ behaves as the canonical projection of $g(G)$ onto $L(G / N)$, as noted above, and $\bar{\eta}$ is evidently a continuous homomorphism

(2) Cf. [9], Theorem 3.11 and Lemma 3.12. 
of $L(G / N)$ into $L\left(G^{\prime} / N^{\prime}\right)$. Hence it follows that $\bar{\pi}_{N^{\prime}}^{G^{\prime} \circ \bar{\theta}}$ is a continuous homomorphism of $\mathrm{g}(G)$ into $L\left(G^{\prime} / N^{\prime}\right)$. Since $N^{\prime}\left(\in \boldsymbol{H}_{0}(G)\right)$ is arbitrary, this just shows that $\bar{\theta}$ is a continuous homomorphism of $g(G)$ into $g\left(G^{\prime}\right)$. The rest of (i) now follows automatically since the locally convex space $g(G)$ is minimal (see [4], Chap. 4 , $§ 1$, exercise 13).

(ii): The first half is obvious from (0.6). Let us see the second half. Let $N^{\prime} \in \boldsymbol{H}_{0}(G)$. Since $\theta(G)=G^{\prime}$ by assumption, the map $\eta$ introduced above carries $G / N$ onto $G^{\prime} / N^{\prime}$ in the present case. Since $G / N$ and $G^{\prime} / N^{\prime}$ are connected Lie groups, this implies that $\bar{\eta}$ maps $L(G / N)$ onto $L\left(G^{\prime} / N^{\prime}\right)$. Hence $\bar{\pi}_{N^{\prime}}^{G^{\prime}} \circ \bar{\theta}\left(=\bar{\eta} \circ \bar{\pi}_{N}^{G}\right)$ maps $\mathrm{g}(G)$ onto $L\left(G^{\prime} / N^{\prime}\right)$. Since $N^{\prime}$ is arbitrary, this shows that $\bar{\theta}(\mathrm{g}(G))$ is dense in $\mathfrak{g}\left(G^{\prime}\right)$. But, by (i), $\bar{\theta}(\mathfrak{g}(G))$ is closed in $\mathfrak{g}\left(G^{\prime}\right)$. Hence $\bar{\theta}(\mathfrak{g}(G))=\mathfrak{g}\left(G^{\prime}\right)$. q.e.d.

Corollary 1. Isomorphic LC groups have the same Lie algebra.

Corollary 2. $\bar{\theta}(L(G)) \cong L(G) / \operatorname{ker} \bar{\theta}$ (as topological Lie algebra). Here $\operatorname{ker} \bar{\theta}$ is given by (0.6).

If $R(G), R\left(G^{\prime}\right)$ are employed for $L(G), L\left(G^{\prime}\right)$ according to our convention, then

$$
\bar{\theta}(\alpha)=\theta \circ \alpha \quad(\alpha \in R(G))
$$

Hence,

Corollary 3. If $\theta(c(G))=c\left(G^{\prime}\right)$, then $R\left(G^{\prime}\right)=\{\theta \circ \alpha ; \alpha \in R(G)\}$.

0.4. Lie algebra of subgroups and quotient groups. For any closed subgroup $H$ of $G$, put

$$
L_{H}(G)=\left\{X \in L(G) ; \exp _{G} t X \in H \text { for all } t \in \boldsymbol{R}\right\} .
$$

(Note that the right side of $(0.6)$ is now designated as $L_{\text {ker } \theta}(G)$.) Plainly, $R(H)$ consists of all elements of $R(G)$ with orbit in $H$. Therefore, under our isomorphy between $L(G)$ and $R(G), L_{H}(G)$ and $R(H)$ are in correspondence.

Lemma 0.7. Let $H$ be a closed subgroup of $G$, and $j$ the canonical injection of $H$ into $G$.

(i) $L_{H}(G)$ is a closed Lie subalgebra of $L(G)$ isomorphic to $L(H)$ under $\bar{j}$.

(ii) If $H$ is normal in $G$, then $L_{H}(G)$ is an ideal of $L(G)$.

(iii) If $H$ is compact and normal in $G$, then

$$
\begin{aligned}
& L(G / H) \cong L(G) / L_{H}(G) \quad \text { (as topological Lie algebra), } \\
& R(G / H)=\left\{\pi_{H}^{G} \circ \alpha ; \alpha \in R(G)\right\} .
\end{aligned}
$$

Proof. (i) is easily seen from $j\left(\exp _{H} t Y\right)=\exp _{G} t \bar{j}(Y)(t \in \boldsymbol{R}, Y \in L(H))$ and Lemma 0.6. If $H$ is normal, then $L_{H}(G)=\operatorname{ker} \bar{\pi}_{H}^{G}$ by (0.6). Hence (ii). If, furthermore, $H$ is compact, then $\pi_{H}^{G}(c(G))=c(G / H)$ (Lemma 0.2). Therefore 
Lemma 0.6 and Corollaries 2 and 3 to it yield (iii). q. e. d.

Note. Let $H$ be a closed normal subgroup of $G$, and either $G$ or $H$ be connected. Then, even if $H$ is not compact, $\pi_{H}^{G}(c(G))=c(G / H)$ holds, and hence (0.9) and (0.10) also hold. But this fact is not used in the sequel.

Lemma 0.8. Suppose $G$ is Lie-projective. For each neighbourhood $C V$ of 0 in $R(G)$, there exists an $\left.N \in \boldsymbol{H}_{0} G\right)$ s.t. $R(N) \subseteq \mathcal{C}$.

Proof. It suffices to see that each neighbourhood $C V$ of 0 in $L(G)$ includes $L_{N}(G)$ for some $N \in \boldsymbol{H}_{0}(G)$. But this is clear since $L_{N}(G)=\operatorname{ker} \bar{\pi}_{N}^{G}$ and $\bar{\pi}_{N}^{G}$ behaves as the canonical projection of $\mathfrak{g}(G)$ onto $L(G / N)$. q. e.d.

\subsection{Adjoint representation.}

Definition 0.5. For each $x \in G, \operatorname{Ad}_{G}(x)$ denotes the differential of the inner automorphism $y \mapsto x y x^{-1}$ of $G$.

That is, $\operatorname{Ad}_{G}(x)$ is determined by $x\left(\exp _{G} t X\right) x^{-1}=\exp _{G} t \operatorname{Ad}_{G}(x) X(t \in \boldsymbol{R}, X \in$ $L(G)$ ). In view of Lemma 0.6, $\operatorname{Ad}_{G}(x)$ is a topological automorphism of $L(G)$. The $\operatorname{map} \operatorname{Ad}_{G}: x \in G \mapsto \operatorname{Ad}_{G}(x)$ gives a linear representation of $G$ on $L(G)$ in the algebraic sense.

Lemma 0.9. For each $X \in L(G)$, the map $x \mapsto \operatorname{Ad}_{G}(x) X$ of $G$ into $L(G)$ is continuous.

Proof. First suppose $G$ is Lie-projective. Realize $L(G)$ by $g(G)$. For $X=$ $\left(X_{N}\right)_{N \in H_{0}(G)} \in \mathfrak{g}(G)$ and $t \in \boldsymbol{R}$, we have

$$
\begin{aligned}
& \iota_{G}\left(\exp _{G} t \operatorname{Ad}_{G}(x) X\right)=\iota_{G}\left(x\left(\exp _{G} t X\right) x^{-1}\right) \\
& =\left(x_{N}\left(\exp _{G / N} t X_{N}\right) x_{N}^{-1}\right)_{N \in H_{0}(G)} \quad(\text { by }(0.1) \text { and }(0.4)) \\
& =\left(\exp _{G / N} t \operatorname{Ad}_{G / N}\left(x_{N}\right) X_{N}\right)_{N \in H_{0}(G)}
\end{aligned}
$$

where $x_{N}=\pi_{N}^{G}(x)$, and each $\operatorname{Ad}_{G / N}$ denotes the usual adjoint representation of the Lie group $G / N$. Hence, again by $(0.1)$ and $(0.4), \bar{\pi}_{N}^{G}\left(\operatorname{Ad}_{G}(x) X\right)=\operatorname{Ad}_{G / N}\left(x_{N}\right) X_{N}$ for all $N \in \boldsymbol{H}_{0}(G)$. Thus

$$
\operatorname{Ad}_{G}(x) X=\left(\operatorname{Ad}_{G / N}\left(x_{N}\right) X_{N}\right)_{N \in H_{0}(G)} .
$$

This evidently shows that $\operatorname{Ad}_{G}(x) X$ depends on $x$ continuously.

Next let $G$ be arbitrary. Take a Lie-projective open subgroup $G_{1}$ of $G$ (see 0.1). We have only to show that $\operatorname{Ad}_{G}(x) X$ is continuous on each right $G_{1^{-}}$. coset $G_{1} x_{0}$ of $G$. Since $c\left(G_{1}\right)=c(G)$ and so $L\left(G_{1}\right)=L(G)$, we have $\exp _{G}=\exp _{G_{1}}$. Hence $\operatorname{Ad}_{G}(x)=\operatorname{Ad}_{G_{1}}(x)$ if $x \in G_{1}$. Therefore, for $x \in G_{1}$,

$$
\operatorname{Ad}_{G}\left(x x_{0}\right) X=\operatorname{Ad}_{G_{1}}(x)\left(\operatorname{Ad}_{G}\left(x_{0}\right) X\right) .
$$

By what has already been shown, this depends on $x\left(\in G_{1}\right)$ continuously. Thus 
the lemma has been proved. q.e.d.

Since $L(G)$ is barrelled, we have the following

Corollary. The representation $\operatorname{Ad}_{G}$ of $G$ on $L(G)$ is continuous (i.e., the map $(x, X) \in G \times L(G) \mapsto \operatorname{Ad}_{G}(x) X \in L(G)$ is continuous).

It is needless to say that $\operatorname{Ad}_{G}(x)(x \in G)$ acts on $R(G)$ as $\operatorname{Ad}_{G}(x) \alpha=x \alpha x^{-1}$ $(\alpha \in R(G))$, where $x \alpha x^{-1}(\in R(G))$ is defined as $\left(x \alpha x^{-1}\right)(t)=x \alpha(t) x^{-1}(t \in R)$.

0.6. The dimension of $G$. By dimension of a compact space we mean its covering dimension (e.g. [11], §16). The next lemma is elementary.

Lemma 0.10. Let $S$ be a compact Hausdorff space. (i) The dimension of a closed subset of $S$ does not exceed that of $S$. (ii) Let $A, B$ be closed subsets of $S$ s.t. $A \cup B=S$. Then the dimension of $S$ equals the greater of the dimensions of $A$ and $B$. (iii) Let $Z$ be a 0 -dimensional (i.e., totally disconnected) compact Hausdorff space. Then the dimension of the product space $S \times Z$ equals that of $S$.

Let $A, B$ be any two compact subsets of $G$ with non-void interior. Then each of them is covered by a finite number of translations of the other. Therefore, by (i) and (ii) of Lemma 0.10 , one sees that they have the same dimension. So we make the following

Definition 0.6. The identical dimension of all compact subsets of $G$ with non-void interior is called the dimension of $G$.

This definition is consistent with the dimension of a Lie group in the usual sense. Indeed, an $n$-dimensional Lie group contains an $n$-cell as a compact neighbourhood of its identity.

As for the proof of the next lemma, see the verification of [11], Theorem 69.

Lemma 0.11. Suppose $N \in H_{0}(G)$. Then there exists a subset $L$ of $G$ fulfilling the following three conditions: (i) $e \in L$; (ii) $L$ is homeomorphic under $\pi_{N}^{G}$ with an open neighbourhood of $\pi_{N}^{G}(e)$ in $G / N$ (hence $L N$ is an open neighbourhood of $e$ in $G)$; (iii) the map $(y, z) \in L \times N \mapsto y z \in L N$ is a homeomorphism. Besides, if $N$ is totally disconnected, such an $L$ can be chosen so as to satisfy one more condition: (iv) the map $\left(\pi_{N}^{G}(y), z\right) \in \pi_{N}^{G}(L) \times N \mapsto y z \in L N$ is a local isomorphism of $(G / N) \times N$ into $G$.

Lemma 0.12. Let $d(G)$ be the supremum (finite or infinite) of the dimensions of all cells in $G$. Take a pro-Lie open subgroup $G_{1}$ of $G$ (see 0.1 ) and put $r\left(G_{1}\right)$ $=\sup \left\{\operatorname{dim} G_{1} / N ; N \in \boldsymbol{H}_{0}\left(G_{1}\right)\right\}$. Then

$$
\operatorname{dim} G=d(G)=r\left(G_{1}\right)=\operatorname{dim} L(G) .
$$

Proof. (I) First we prove $\operatorname{dim} G=d(G)=r\left(G_{1}\right)$ by showing $\operatorname{dim} G \geqq d(G) \geqq$ 
$d\left(G_{1}\right) \geqq r\left(G_{1}\right) \geqq \operatorname{dim} G_{1}=\operatorname{dim} G$. Each cell in $G$ is covered by a finite number of translations of a compact neighbourhood of $e$. Hence, by (i) and (ii) of Lemma 0.10 , one has $\operatorname{dim} G \geqq d(G) . \quad d(G) \geqq d\left(G_{1}\right)$ and $\operatorname{dim} G_{1}=\operatorname{dim} G$ are obvious. Furthermore, in view of the condition (ii) of Lemma $0.11, d\left(G_{1}\right) \geqq \operatorname{dim} G_{1} / N$ holds for any $N \in \boldsymbol{H}_{0}(G)$. Hence $d\left(G_{1}\right) \geqq r\left(G_{1}\right)$. Therefore it remains now only to show $r\left(G_{1}\right) \geqq \operatorname{dim} G_{1}$. To do so we can assume that $r\left(G_{1}\right)<\infty$. Then there exists an $N_{0} \in \boldsymbol{H}_{0}\left(G_{1}\right)$ s.t. $\operatorname{dim} G_{1} / N_{0}=r\left(G_{1}\right)$. We now show that $N_{0}$ is totally disconnected (cf. [10], p. 182). Suppose $N \in \boldsymbol{H}_{0}\left(G_{1}\right)$ and $N \subseteq N_{0}$. Then $\left(G_{1} / N\right) /\left(N_{0} / N\right) \cong G_{1} / N_{0}$. Since $G_{1} / N$ is a Lie group and $N_{0} / N$ is its closed normal subgroup, this demands by the choice of $N_{0}$ that $N_{0} / N$ is discrete. Hence $N$ is open in $N_{0}$. Therefore $c\left(N_{0}\right) \subseteq N$. Since $G_{1}$ is Lie-projective, $N$ can be chosen arbitrarily small. Thus, after all, $c\left(N_{0}\right)=\{e\}$ (i.e., $N_{0}$ is totally disconnected). By Lemma $0.11 G_{1}$ is now locally isomorphic to $\left(G_{1} / N_{0}\right) \times N_{0}$. Therefore, by (iii) of Lemma 0.10 , we see that $G_{1} / N_{0}$ and $G_{1}$ have the same dimension (i.e., $r\left(G_{1}\right)=\operatorname{dim} G_{1}$ ).

(II) Next we prove $r\left(G_{1}\right)=\operatorname{dim} L(G)$. Since $L(G)=L\left(G_{1}\right)$, it suffices to show $r\left(G_{1}\right)=\operatorname{dim} L\left(G_{1}\right)$. For any $N \in \boldsymbol{H}_{0}(G)$, one has $L\left(G_{1} / N\right) \cong L\left(G_{1}\right) / L_{N}\left(G_{1}\right)((0.9))$. Since $G_{1} / N$ is a Lie group, it thus follows that $\operatorname{dim} G_{1} / N=\operatorname{dim} L\left(G_{1} / N\right) \leqq$ $\operatorname{dim} L\left(G_{1}\right)$. Hence $r\left(G_{1}\right) \leqq \operatorname{dim} L\left(G_{1}\right)$. For the proof of the reverse inequality, we can assume that $r\left(G_{1}\right)<\infty$. There exists in this case a totally disconnected $N \in \boldsymbol{H}_{0}\left(G_{1}\right)$ (see (I)). Then, since $L_{N}\left(G_{1}\right) \cong L(N)=\{0\}$ (Lemma 0.7 ), one has $\operatorname{dim} L\left(G_{1}\right)=\operatorname{dim} L\left(G_{1} / N\right)=\operatorname{dim} G_{1} / N \leqq r\left(G_{1}\right)$, completing the proof. q. e.d.

In (I) of the above proof we verified incidentally the following

Lemma 0.13. Suppose $G$ is finite-dimensional and Lie-projective. Then there exists a totally disconnected $N \in \boldsymbol{H}_{0}(G)$.

A well known consequence of Lemmas 0.11 and 0.13 is this : a finite-dimensional $L C$ group is a Lie group if and only if it is locally connected.

\subsection{Compact normal subgroups with finite co-dimension.}

Definition 0.7. $\boldsymbol{H}(G)$ denotes the totality of compact normal subgroups $N$ of $G$ such that $G / N$ is finite-dimensional. $\boldsymbol{H}_{1}(G)$ denotes the subset of $\boldsymbol{H}(G)$ consisting of all $N \in \boldsymbol{H}(G)$ such that $G / N$ satisfies the first countability axiom.

Needless to say, one has $\boldsymbol{H}_{0}(G) \cong \boldsymbol{H}_{1}(G)$. If $G$ is almost conneted (hence $\sigma$ compact), $G / N$ for $N \in \boldsymbol{H}_{1}(G)$ is second countable (i.e., first countable and $\boldsymbol{\sigma}$ compact). Note that if $G$ is finite-dimensional, $\boldsymbol{H}(G)$ consists of all compact normal subgroups of $G$, containing in particular the subgroup $\{e\}$.

Lemma 0.14. Suppose $G$ is Lie-projective. Then, for a compact normal subgroup $N$ of $G$, the following three statements are equivalent: (a) $N \in \boldsymbol{H}(G)$; (b) $c(N) \in \boldsymbol{H}(G)$; (c) $c\left(N_{0}\right) \subseteq N$ for some $N_{0} \in H_{0}(G)$. 
Proof. The verification is the same as in the compact case (see [6], Lemma 3.1) if we keep Lemmas 0.7 and 0.13 above in mind. q.e.d.

Since $\boldsymbol{H}_{0}(G)$ is lower directed, this lemma yields the following

Corollary. Let $G$ be Lie-projective. Then $\boldsymbol{H}(G)$ is lower directed and $\{c(N)$; $\left.N \in \boldsymbol{H}_{0}(G)\right\}$ is cofinal in it.

Lemma 0.15. Suppose $G$ is Lie-projective. The following three statements are equivalent: (a) $\boldsymbol{H}(G)=\boldsymbol{H}_{0}(G)$; (b) $c(N) \in \boldsymbol{H}_{0}(G)$ for every $N \in \boldsymbol{H}_{0}(G)$; (c) $G$ is locally connected. ${ }^{(3)}$

Proof. See the proof of [6], Lemma 3.2. q.e.d.

Lemma 0.16. (i) If $G_{1}, G_{2}$ are pro-Lie open subgroups of $G$, then so is $G_{1} \cap G_{2}$.

(ii) Let $G_{1}, G_{2}$ be pro-Lie open subgroups of $G$ s.t. $G_{1} \cong G_{2}$. Then $\{N \in$ $\left.\boldsymbol{H}_{0}\left(G_{2}\right) ; N \leqq G_{1}\right\}$ (resp. $\left.\left\{N \in \boldsymbol{H}\left(G_{2}\right) ; N \leqq G_{1}\right\}\right)$ is cofinal in both of $\boldsymbol{H}_{0}\left(G_{1}\right)$ and $\boldsymbol{H}_{0}\left(G_{2}\right)$ (resp. $\boldsymbol{H}\left(G_{1}\right)$ and $\boldsymbol{H}\left(G_{2}\right)$ ).

Proof. (i) is obvious. (ii) follows from Lemmas 0.3 and 0.14 . q.e.d.

\section{Chapter 1. Spaces of differentiable functions}

1.1. The " $C^{n}$-classes" on $G(n=\infty, 1,2, \cdots)$. Riss [12] introduced the notion of $C^{n}$-classes on abelian $L C$ groups. Its generalization to the non-abelian case was attained in the book [3] by H. Boseck, G. Czichowski and K. P. Rudolph. In this section we sketch this generalization in somewhat modified arguments in parallel with our treatment of the compact case in [6].

Definition 1.1. Let $f$ be a $C$-valued function on $G$ and $\alpha \in R(G)$. Suppose the function $t \in \boldsymbol{R} \mapsto f(x \alpha(t))$ (resp. $f(\alpha(-t) x)$ ) is differentiable at 0 for each $x \in G$.

In this case we define the function $d_{\alpha}^{(r)} f$ (resp. $d_{\alpha}^{(l)} f$ ) on $G$ as

$$
\begin{gathered}
d_{\alpha}^{(r)} f(x)=\left.\frac{d}{d t} f(x \alpha(t))\right|_{t=0} \\
\text { (resp. } \left.d_{\alpha}^{(l)} f(x)=\left.\frac{d}{d t} f(\alpha(-t) x)\right|_{t=0}\right) \quad(x \in G),
\end{gathered}
$$

and call it the right (resp. left) derivative of $f$ with respect to $\alpha$.

Definition 1.2. $\mathcal{E}_{0}(G)$ denotes the set of all $C$-valued continuous functions on $G$. For each $n=1,2,3, \cdots, \mathcal{E}_{n}^{(r)}(G)$ denotes the set of all functions $f$ in $\mathcal{E}_{0}(G)$ such that the right derivatives of higher order $d_{\alpha_{k}}^{(r)} \cdots d_{\alpha_{1}}^{(r)} f$ exist and belong to $\mathcal{E}_{0}(G)$ for any $\alpha_{1}, \cdots, \alpha_{k} \in R(G)$ with $1 \leqq k \leqq n$ (hence $\mathcal{E}_{n}^{(r)}(G) \supseteqq \mathcal{E}_{n+1}^{r)}(G)$ ). We put $\mathcal{E}_{\infty}^{(r)}(G)=\cap\left\{\mathcal{E}_{n}^{(r)}(G) ; n=1,2,3, \cdots\right\}$. For each $n=1,2,3, \cdots, \infty$, the set

(3) Cf. [2], Proposition 5. 
$\mathcal{E}_{n}^{(l)}(G)$ is defined similarly using the left derivatives instead of the right ones.

Suppose $G$ is abelian (resp. a Lie group). Then, of course, $\mathcal{E}_{n}^{(r)}(G)$ and $\mathcal{E}_{n}^{(l)}(G)$ coincide with each other for each $n(=\infty, 1,2, \cdots)$ and give the $C^{n}$-class on $G$ in Riss' (resp. the usual) sense. We now intend to prove this coincidence for any $G$.

The first lemma is straightforward.

Lemma 1.1. (i) Let $f, g \in \mathcal{E}_{1}^{(r)}(G)$. Then $a f+b g(a, b \in \boldsymbol{C}), f g, \bar{f}, x_{0} f$, and $f_{x_{0}}\left(x_{0} \in G\right)$ belong to $\mathcal{E}_{1}^{(r)}(G)$. And, for $\alpha \in R(G)$, there hold

$$
\begin{aligned}
& d_{\alpha}^{(r)}(a f+b g)=a d_{\alpha}^{(r)} f+b d_{\alpha}^{(r)} g, \\
& d_{\alpha}^{(r)}(f g)=\left(d_{a}^{(r)} f\right) g+f\left(d_{\alpha}^{(r)} g\right), \\
& d_{\alpha}^{(r)} \bar{f}=\left(d_{\alpha}^{(r)} f\right)^{-}, \\
& d_{\alpha}^{(r)}\left(x_{0} f\right)={ }_{x_{0}}\left(d_{\alpha}^{(r)} f\right), \\
& d_{\alpha}^{(r)}\left(f_{x_{0}}\right)=\left(d_{x_{0}^{-1} \alpha x_{0}}^{(r)} f\right)_{x_{0}} .
\end{aligned}
$$

The corresponding facts hold also for the functions in $\mathcal{E}_{1}^{(l)}(G)$.

(ii) A $\boldsymbol{C}$-valued function $f$ on $G$ belongs to $\mathcal{E}_{1}^{(r)}(G)$ if and only if $\check{f} \in \mathcal{E}_{1}^{(l)}(G)$. In this case, for $\alpha \in R(G)$,

$$
d_{\alpha}^{(l)} \check{f}=\left(d_{\alpha}^{(r)} f\right)^{\check{r}} .
$$

Corollary. Each of $\mathcal{E}_{n}^{(r)}(G)$ and $\mathcal{E}_{n}^{(l)}(G)(n=\infty, 1,2, \cdots)$ is an algebra over $\boldsymbol{C}$ under the obvious algebraic operations, and stable under the left and the right translations and the complex conjugation. The inversion $f \mapsto \check{f}$ induces an algebra isomorphism between $\mathcal{E}_{n}^{(r)}(G)$ and $\mathcal{E}_{n}^{(l)}(G)$.

Definition 1.3. For each $C \in \operatorname{Com}(G), \operatorname{Com}(G)$ being the set of all compact subsets of $G$, define a seminorm $P_{C}$ on the $C$-linear space $\mathcal{E}_{0}(G)$ as

$$
P_{C}(f)=\sup _{x \in C}|f(x)| \quad\left(f \in \mathcal{E}_{0}(G)\right) .
$$

We topologize $\mathcal{E}_{0}(G)$ by $\left\{P_{C} ; C \in \operatorname{Com}(G)\right\}$. For any $C \in \operatorname{Com}(G)$ and $\alpha_{1}, \cdots, \alpha_{k}$ $\in R(G)(k=1,2,3, \cdots)$, define a seminorm $P_{C ; \alpha_{1}, \cdots, \alpha_{k}}$ (resp. $\left.P_{C}^{\alpha_{1}, \cdots, \alpha_{k}}\right)$ on the $C$ linear space $\mathcal{E}_{k}^{(r)}(G)$ (resp. $\mathcal{E}_{k}^{(l)}(G)$ ) as

$$
\begin{array}{rr}
P_{C, \alpha_{1}, \cdots, \alpha_{k}}(f)=P_{C}\left(d_{\alpha_{1}}^{(r)} \cdots d_{\alpha_{k}}^{(r)} f\right) & \left(f \in \mathcal{E}_{k}^{(r)}(G)\right) \\
\text { (resp. } \left.P_{C}^{\alpha}, \cdots, \alpha_{k}(f)=P_{C}\left(d_{\alpha_{1}}^{(l)} \cdots d_{\alpha_{k}}^{(l)} f\right) \quad\left(f \in \mathcal{E}_{k}^{(l)}(G)\right)\right) .
\end{array}
$$

We topologize each $\mathcal{E}_{n}^{(r)}(G)$ (resp. $\left.\mathcal{E}_{n}^{(l)}(G)\right)(n=\infty, 1,2, \cdots)$ by the family

$$
\widetilde{\mho}_{n}^{(r)}=\left\{P_{C}, P_{C, \alpha_{1}, \cdots, \alpha_{k}} ; C \in \operatorname{Com}(G), \alpha_{1}, \cdots, \alpha_{k} \in R(G)(1 \leqq k<n+1)\right\}
$$

$\left(\right.$ resp. $\left.\mathfrak{F}_{n}^{(l)}=\left\{P_{C}, P_{C}^{\alpha_{1}, \cdots, \alpha_{k}} ; C \in \operatorname{Com}(G), \alpha_{1}, \cdots, \alpha_{k} \in R(G)(1 \leqq k<n+1)\right\}\right)$

For each compact subgroup $N$ of $G$, we define the continuous peojections (i. e., idempotent linear maps) $\rho_{N}$ and $\sigma_{N}$ on $\mathcal{E}_{0}(G)$ as 


$$
\begin{aligned}
& \rho_{N}(f)(x)=\int_{N} f(x y) d_{N} y, \\
& \sigma_{N}(f)(x)=\int_{N} f(y x) d_{N} y \quad\left(f \in \mathcal{E}_{0}(G), x \in G\right) .
\end{aligned}
$$

where $d_{N}$ denotes the Haar measure on $N$ s.t. $\int_{N} d_{N}=1$. If $N$ is normal, these two coincide with each other. One has $\rho_{N} \rho_{N^{\prime}}=\rho_{N^{\prime}} \rho_{N}=\rho_{N^{\prime}}$ if $N \cong N^{\prime}$. Differentiation under the integral sign immediately shows that $\rho_{N}$ (resp. $\sigma_{N}$ ) induces a continuous projection on each $\mathcal{E}_{n}^{(l)}(G)\left(\operatorname{resp} . \mathcal{E}_{n}^{(r)}(G)\right)(n=\infty, 1,2, \cdots)$.

Lemma 1.2. Let $\boldsymbol{H}$ be a lower directed family of compact subgroups of $G$ such that $\bigcap\{N \in \boldsymbol{H}\}=\{e\}$. Then, for each $f \in \mathcal{E}_{n}^{(r)}(G)\left(\right.$ resp. $\left.\mathcal{E}_{n}^{(l)}(G)\right)(n=\infty, 1,2, \cdots)$, the net $\left\{\sigma_{N}(f) ; N \in \boldsymbol{H}\right\}$ (resp. $\left\{\rho_{N}(f) ; N \in \boldsymbol{H}\right\}$ ) converges to $f$ in $\mathcal{E}_{n}^{(r)}(G)$ (resp. $\left.\mathcal{E}_{n}^{(l)}(G)\right)$.

Proof. For any $C \in \operatorname{Com}(G)$ and $\alpha_{1}, \cdots, \alpha_{k} \in R(G)(0 \leqq k<n+1)$, we have

$$
\begin{aligned}
& P_{C, \alpha_{1}, \cdots, \alpha_{k}}\left(\sigma_{N}(f)-f\right) \\
& \quad=\sup _{x \in C}\left|\int_{N}\left\{d_{\alpha_{1}}^{(r)} \cdots d_{\alpha_{k}}^{(r)} f(y x)-d_{\alpha_{1}}^{(r)} \cdots d_{\alpha_{n}}^{(r)} f(x)\right\} d_{N} y\right| \\
& \quad \leqq \sup _{x \in C} \sup _{y \in N}\left|d_{\alpha_{1}}^{(r)} \cdots d_{\alpha_{k}}^{(r)} f(y x)-d_{\alpha_{1}}^{(r)} \cdots d_{\alpha_{k}}^{(r)} f(x)\right| .
\end{aligned}
$$

Hence the lemma. q.e.d.

Let $N$ be a compact normal subgroup of $G$. For each $n=\infty, 1,2, \cdots$, let us put

$$
\mathcal{E}_{n}^{(r)}(G, N)=\sigma_{N}\left(\mathcal{E}_{n}^{(r)}(G)\right) \quad\left(\text { resp. } \mathcal{E}_{n}^{(l)}(G, N)=\rho_{N}\left(\mathcal{E}_{n}^{(l)}(G)\right)\right) .
$$

This is identical with $\left\{f \in \mathcal{E}_{n}^{(r)}(G) ;{ }_{y} f=f(y \in N)\right\}$ (resp. $\left\{f \in \mathcal{E}_{n}^{(l)}(G) ; f_{y}=f\right.$ $(y \in N)\})$ and hence a subalgebra of $\mathcal{E}_{n}^{(r)}(G)$ (resp. $\mathcal{E}_{n}^{(l)}(G)$ ). In virtue of $(0.10)$ we have the following

Lemma 1.3. Let $N$ be a compact normal subgroup of $G$. A C-valued function $g$ on $G / N$ belongs to $\mathcal{E}_{1}^{(r)}(G / N)$ (resp. $\mathcal{E}_{1}^{(l)}(G / N)$ ) if and only if $g \circ \pi_{N}^{G} \in$ $\mathcal{E}_{1}^{(r)}(G, N)$ (resp. $\left.\mathcal{E}_{1}^{(l)}(G, N)\right)$. In this case, for $\alpha \in R(G)$,

$$
\begin{aligned}
d_{\alpha}^{(r)}\left(g \circ \pi_{N}^{G}\right) & =\left(d_{\bar{\alpha}}^{(r)} g\right) \circ \pi_{N}^{G} \\
\left(\text { resp. } d_{\alpha}^{(l)}\left(g \circ \pi_{N}^{G}\right)\right. & \left.=\left(d_{\bar{\alpha}}^{(l)} g\right) \circ \pi_{N}^{G}\right),
\end{aligned}
$$

where $\bar{\alpha}=\bar{\pi}_{N}^{G}(\alpha)$.

Corollary. Let $N$ be as above. For each $n=\infty, 1,2, \cdots$, the map $g \mapsto g \circ \pi_{N}^{G}$ sets up an algebra isomorphism of $\mathcal{E}_{n}^{(r)}(G / N)$ (resp. $\mathcal{E}_{n}^{(l)}(G / N)$ ) onto $\mathcal{E}_{n}^{(r)}(G, N)$ (resp. $\left.\mathcal{E}_{n}^{(l)}(G, N)\right)$. 
Lemma 1.4. Let $f \in \mathcal{E}_{1}^{(r)}(G)$ (resp. $\mathcal{E}_{1}^{(l)}(G)$ ). Then the map $\alpha \mapsto d_{\alpha}^{(r)} f$ (resp. $\left.d_{\alpha}^{(l)} f\right)$ of $R(G)$ into $\mathcal{E}_{0}(G)$ is $\boldsymbol{R}$-linear.

Proof. (I) First suppose $G$ is Lie-projective. For each $N \in \boldsymbol{H}_{0}(G)$, put $f_{N}=\sigma_{N}(f)$ and take $g_{N} \in \mathcal{E}_{1}^{(r)}(G / N)$ s.t. $f_{N}=g_{N} \circ \pi_{N}^{G}$ (Lemma 1.3). Then, since $\mathcal{E}_{1}^{(r)}(G / N)$ is the usual $C^{n}$-class on the Lie group $G / N$, we have, for $\alpha, \beta \in R(G)$ and $a, b \in \boldsymbol{R}$,

$$
\begin{aligned}
d_{a \alpha+b \beta}^{(r)} f_{N} & =\left(d_{a \bar{\alpha}+b \bar{\beta}}^{(r)} g_{N}\right) \circ \pi_{N}^{G}=\left(a d_{\bar{\alpha}}^{(r)} g_{N}+b d_{\bar{\beta}}^{(r)} g_{N}\right) \circ \pi_{N}^{G} \\
& =a d_{\alpha}^{(r)} f_{N}+b d_{\beta}^{(r)} f_{N} .
\end{aligned}
$$

Since the net $\left\{f_{N} ; N \in \boldsymbol{H}_{0}(G)\right\}$ converges to $f$ in $\mathcal{E}_{1}^{(r)}(G)$ (Lemma 1.2), this yields the equality $d_{a \alpha+b \beta}^{(r)}=f=a d_{\alpha}^{(r)} f+b d_{\beta}^{(r)} f$.

(II) Next let $G$ be arbitrary. Take a pro-Lie open subgroup $G_{1}$ of $G$. Then, since $R\left(G_{1}\right)=R(G)$ and, for each $x \in G$, the restriction of ${ }_{x} f$ to $G_{1}$ belongs to $\mathcal{E}_{1}^{(r)}\left(G_{1}\right)$, we have by (I)

$$
\begin{aligned}
d_{a(1+b \beta}^{(r)} f(x) & =d_{a \alpha+b \beta}^{(r)}\left({ }_{x} f\right)(e) \quad(\text { Lemma 1.1, (i)) } \\
& =a d_{\alpha}^{(r)}\left({ }_{x} f\right)(e)+b d_{\beta}^{(r)}\left({ }_{x} f\right)(e) \\
& =a d_{\alpha}^{(r)} f(x)+b d_{\beta}^{(r)} f(x),
\end{aligned}
$$

completing the proof. q.e.d.

Remark 1.1. In the same way as above we can prove

$$
d_{[\alpha, \beta]}^{(r)} f=d_{\alpha}^{(r)} d_{\beta}^{(r)} f-d_{\beta}^{(r)} d_{\alpha}^{(r)} f \quad\left(\alpha, \beta \in R(G), f \in \mathcal{E}_{2}^{(r)}(G)\right) .
$$

Lemma 1.5. For each $f \in \mathcal{E}_{1}^{(r)}(G)$ (resp. $\mathcal{E}_{1}^{(l)}(G)$ ) and $x \in G$, the map $\alpha \mapsto$ $d_{\alpha}^{(r)} f(x)$ (resp. $d_{\alpha}^{(l)} f(x)$ ) of $R(G)$ into $\boldsymbol{C}$ is $\boldsymbol{R}$-linear and continuous.

Proof. See the proof of [6], Lemma 2.8, having in mind Lemma 1.4 above and the fact that the map $\alpha \in R(G) \mapsto \alpha(t) \in G$ is continuous, $t$ being fixed (see 0.2). q.e.d.

The next lemma now follows from Lemma 1.5 and the barrelledness of $R(G)$ (cf. Proof of [6], Lemma 2.9).

Lemma 1.6 ([3], Proposition 2.2.1.2). Let $f \in \mathcal{E}_{1}^{(r)}(G)$ (resp. $\mathcal{E}_{1}^{(l)}(G)$ ). The map $(\alpha, x) \mapsto d_{\alpha}^{(r)} f(x)$ (resp. $d_{\alpha}^{(l)} f(x)$ ) of $R(G) \times G$ into $C$ is continuous.

Theorem 1.1. For each $n=\infty, 1,2, \cdots$, the sets $\mathcal{E}_{n}^{(r)}(G)$ and $\mathcal{E}_{n}^{(l)}(G)$ coincide with each other.

Proof. For a function $f$ on $G$, we have $f(x \alpha(t))=f\left(x \alpha(t) x^{-1} x\right)(\alpha \in R(G)$, $x \in G)$. Hence it is plain that $d_{\alpha}^{(r)} f$ exists for every $\alpha \in R(G)$ if and only if so with $d_{\alpha}^{(l)} f$. And, in this case, $d_{\alpha}^{(r)} f(x)=-d_{x \alpha x-1}^{(l)} f(x)$ holds. Therefore, by Lemmas 0.9 and 1.6 , one sees that $f$ belongs to $\mathcal{E}_{1}^{(r)}(G)$ if and only if it belongs 
to $\mathcal{E}_{1}^{(l)}(G)$. That is, $\mathcal{E}_{1}^{(r)}(G)=\mathcal{E}_{1}^{(l)}(G)$ holds. The verification of $\mathcal{E}_{n}^{(r)}(G)=\mathcal{E}_{n}^{(l)}(G)$ for $n \geqq 2$ is then attained in the same way as in the compact case. For details, see [6], Lemma 2.12 and what follows it. q.e.d.

On the basis of the above theorem we generalize the notion of $C^{n}$-classes to any $G$ as follows.

Definition 1.4. For each $n=\infty, 1,2, \cdots$, the identical sets $\mathcal{E}_{n}^{(r)}(G)$ and $\mathcal{E}_{n}^{(l)}(G)$ are denoted by $\mathcal{E}_{n}(G)$. Each element of $\mathcal{E}_{n}(G)$ is called an $n$-times (indefinitely if $n=\infty$ ) continuously differentiable function on $G$.

By Corollary to Lemma 1.1 , each $\mathcal{E}_{n}(G)$ is an algebra over $C$ stable under the left and the right translations, the inversion and the complex conjugation. The reader should notice that by virtue of the very definition of $\mathcal{E}_{n}(G)$ based on Theorem 1.1, the mixed derivatives $d_{\alpha_{p}}^{(r)} \cdots d_{\alpha_{1}}^{(r)} d_{\beta_{q}}^{(l)} \cdots d_{\beta_{1}}^{(l)} f$ for $f \in \mathcal{E}_{p+q}(G)$ $(p, q=0,1,2, \cdots)$ can be made. Here, as is easily seen, the "operators" $d_{\alpha}^{(r)}$ and $d_{\beta}^{(l)}$ commute on $\mathcal{E}_{2}(G)$ :

$$
d_{\alpha}^{(r)} d_{\beta}^{(l)} f=d_{\beta}^{(l)} d_{\alpha}^{(r)} f \quad\left(f \in \mathcal{E}_{2}(G), \alpha, \beta \in R(G)\right) .
$$

The Bruhat space $\mathcal{E}(G)$ is included in $\mathcal{E}_{\infty}(G)$. As for the detail of their connection, see 1.5 below.

Definition 1.5. For each $n=\infty, 1,2, \cdots, \mathscr{D}_{n}(G)$ denotes the ideal of the algebra $\mathcal{E}_{n}(G)$ consisting of all $f \in \mathcal{E}_{n}(G)$ with compact support.

For any compact subgroup $N$ of $G$, we put

$$
\begin{aligned}
& \mathcal{E}_{n}(G, N)_{\rho}=\rho_{N}\left(\mathcal{E}_{n}(G)\right) \quad\left(=\left\{f \in \mathcal{E}_{n}(G) ; f_{y}=f(y \in N)\right\}\right), \\
& \mathcal{E}_{n}(G, N)_{\sigma}=\sigma_{. N}\left(\mathcal{E}_{n}(G)\right) \quad\left(=\left\{f \in \mathcal{E}_{n}(G) ;{ }_{y} f=f(y \in N)\right\}\right), \\
& \mathcal{E}_{n}(G, N)=\mathcal{E}_{n}(G, N)_{\rho} \cap \mathcal{E}_{n}(G, N)_{\sigma} \quad(n=\infty, 1,2, \cdots) .
\end{aligned}
$$

These are subalgebras of $\mathcal{E}_{n}(G)$, and coincide with one another if $N$ is normal. Furthermore let us put

$$
\begin{aligned}
& \mathscr{D}_{n}(G, N)_{o}=\mathscr{D}_{n}(G) \cap \mathcal{E}_{n}(G, N)_{o}, \\
& \mathscr{D}_{n}(G, N)_{\sigma}=\mathscr{D}_{n}(G) \cap \mathcal{E}_{n}(G, N)_{\sigma}, \\
& \mathscr{D}_{n}(G, N)=\mathscr{D}_{n}(G) \cap \mathcal{E}_{n}(G, N) .
\end{aligned}
$$

The next theorem generalizes [12], p. 57, Theorem 2 to the non-abelian case (also see [1], I, Satz 4.4 and [3], Theorem 2.2.2.6). We omit the verification since it is the same as the proof of [6], Lemma 3.3 if we use our previous Lemmas $0.5,0.8,0.14$ and 1.5 and recall $R(G)=R\left(G_{1}\right)$.

Theorem 1.2. Let $G_{1}$ be any pro-Lie open subgroup of $G$. If a subset $\mathcal{B}$ of $\mathcal{E}_{1}(G)$ satisfies the condition 


$$
\left.\sup _{x \in G, f \in \mathscr{B}}\left|d_{\alpha}^{(r)} f(x)\right|<\infty \quad \text { (resp. } \sup _{x \in G, f \in \mathscr{B}}\left|d_{\alpha}^{(l)} f(x)\right|<\infty\right)
$$

Sor each $\alpha \in R(G)$, then there exists an $N \in \boldsymbol{H}\left(G_{1}\right)$ (see Definition 0.7) s.t. $\mathscr{B} \cong$ $\mathcal{E}_{1}(G, N)_{\rho}\left(\right.$ resp. $\left.\mathscr{B} \subseteq \mathcal{E}_{1}(G, N)_{\sigma}\right)$.

Notice that this theorem, as well as Corollary 1 below, has significance only when $G$ is infinite-dimensional.

Corollary 1. Let $G_{1}$ be as in Theorem 1.2. If a subset $\mathcal{B}$ of $\mathcal{E}_{1}(G)$ satisfies the condition

$$
\sup _{x \in G, f \in \mathscr{B}}\left\{\left|d_{\alpha}^{(r)} f(x)\right|+\left|d_{\alpha}^{(l)} f(x)\right|\right\}<\infty
$$

for each $\alpha \in R(G)$, then there exists an $N \in \boldsymbol{H}\left(G_{1}\right)$ s.t. $\mathscr{B} \cong \mathcal{E}_{1}(G, N)$.

Proof. Obvious since $\boldsymbol{H}\left(G_{1}\right)$ is lower directed. q. e.d.

Corollary 2. Suppose $G$ is almost connected. If a subset $\mathscr{B}$ of $\mathcal{E}_{1}(G)$ is countable and satisfies the condition

$$
\sup _{x \in G, f \in \mathcal{B}}\left|d_{\alpha}^{(r)} f(x)\right|<\infty
$$

for each $\alpha \in R(G)$, then there exists an $N \in \boldsymbol{H}_{1}(G)$ s.t. $\mathscr{B} \subseteq \mathcal{E}_{1}(G, N)$. The same holds even if the left derivative is employed instead of the right one in (*).

Proof. Since $G$ is almost connected and $\mathscr{B}$ is countable, we have a compact normal subgroup $N_{1}$ of $G$ such that $G / N_{1}$ is first countable and $\mathscr{B} \cong \mathcal{E}_{1}\left(G, N_{1}\right)$ ([10], p. 61). On the other hand, since $G$ is Lie-projective, there exists an $N_{2} \in \boldsymbol{H}(G)$ s.t. $\mathscr{B} \leqq \mathcal{E}_{1}\left(G, N_{2}\right)$ )Theorem 1.2). Put $N=N_{1} N_{2}$. Then $N \in \boldsymbol{H}_{1}(G)$ and $\mathscr{B} \subseteq \mathcal{E}_{1}(G, N)$. q. e.d.

Corollary 3. For each $n=\infty, 1,2, \cdots$, there holds $\mathscr{D}_{n}(G)=\bigcup\left\{\mathscr{D}_{n}(G, N)\right.$; $\left.N \in \boldsymbol{H}\left(G_{1}\right)\right\}, G_{1}$ being the same as in Theorem 1.2. If $G$ is almost connected, then $\mathscr{D}_{n}(G)=\bigcup\left\{\mathscr{D}_{n}(G, N) ; N \in \boldsymbol{H}_{1}(G)\right\}$.

Proof. Corollary 1 applied to each sigleton $\{f\}$ of $\mathscr{D}_{1}(G)$ shows that $f \in$ $\mathscr{D}_{1}(G, N)$ for some $N \in \boldsymbol{H}\left(G_{1}\right)$. Hence the first assertion. The second one follows from Corollary 2 similarly. q.e.d.

1.2. Inductive limit topology for the spaces $\mathscr{D}_{n}(G)$. In this section we introduce for the spaces $\mathscr{D}_{n}(G)$ a natural inductive limit topology. We start with elementary topologies for the spaces $\mathcal{E}_{n}(G)$.

Definition 1.6. For any $C \in \operatorname{Com}(G)$ and $\alpha_{1}, \cdots, \alpha_{p}, \beta_{1}, \cdots, \beta_{q} \in R(G)(p, q=$ $0,1,2, \cdots)$, define a seminorm $P_{C, \alpha_{1}, \cdots, \alpha_{p}}^{\beta_{1}, \ldots, \beta_{q}}$ on $\mathcal{E}_{p+q}(G)$ as

$$
P_{C, \alpha_{1}, \cdots, \alpha_{p}}^{\beta_{1}, \ldots, \beta_{q}}(f)=P_{C}\left(d_{\alpha_{1}}^{(r)} \cdots d_{\alpha_{p}}^{(r)} d_{\beta_{1}}^{(l)} \cdots d_{\beta_{q}}^{(l)} f\right) \quad\left(f \in \mathcal{E}_{p+q}(G)\right) .
$$

If $p$ or $q$ equals 0 , this reduces to one of $P_{C, \alpha_{1}, \cdots, \alpha_{p}}, P_{C}^{\beta_{1}, \cdots, \beta_{q}}$ or $P_{C}$ (see Definition 
1.3). For $n=\infty, 1,2, \cdots$, let $\mathfrak{F}_{n}^{(r)}, \widetilde{F}_{n}^{(l)}$ be the same as in Definition 1.3 , and put

$$
\widetilde{\mho}_{n}=\left\{P_{C, \alpha_{1}, \cdots, \alpha_{p}}^{\beta_{1}, \cdots, \beta_{q}} ; C \in \operatorname{Com}(G), \alpha_{1}, \cdots, \alpha_{p}, \beta_{1}, \cdots, \beta_{q} \in R(G)(0 \leqq p+q<n+1)\right\} .
$$

The locally convex Hausdorff topology for $\mathcal{E}_{n}(G)$ defined by $\mathfrak{F}_{n}^{(r)}$, $\mathfrak{F}_{n}^{(l)}$ and $\mathfrak{F}_{n}$ are denoted by $\boldsymbol{\tau}_{r}, \boldsymbol{\tau}_{l}$ and $\boldsymbol{\tau}_{+}$respectively without regard to $n$. The space $\mathcal{E}_{n}(G)$ equipped with them is denoted by $\mathcal{E}_{n}^{(r)}(G), \mathcal{E}_{n}^{(l)}(G)$ and $\mathcal{E}_{n}^{(+)}(G)$ respectively. (This definition agrees nicely with Definition 1.3.) A linear subspace $\mathcal{K}$ of $\mathcal{E}_{n}(G)$ inheriting these topologies is also denoted by $\mathcal{K}^{(r)}, \mathcal{K}^{(l)}$ and $\mathcal{K}^{(+)}$respectively.

$\tau_{+}$is finer than $\boldsymbol{\tau}_{r}$ and $\boldsymbol{\tau}_{l}$. Each of $\mathcal{E}_{n}^{(r)}(G), \mathcal{E}_{n}^{(l)}(G)$ and $\mathcal{E}_{n}^{(+)}(G)$ is complete. By (1.2) we have

$$
P_{C, \alpha_{1}, \cdots, \alpha_{p}}^{\beta_{1}, \ldots, \beta_{q}}(\check{f})=P_{C^{-1,}, \beta_{1}, \cdots, \beta_{q}}^{\alpha_{1}, \ldots, \alpha_{p}}(f) \quad\left(f \in \mathcal{E}_{p+q}(G)\right) .
$$

Therefore the map $f \mapsto \breve{f}$ induces a topological linear automorphism of $\mathcal{E}_{n}^{(+)}(G)$ and also a topological linear isomorphism of $\mathcal{E}_{n}^{(r)}(G)$ onto $\mathcal{E}_{n}^{(l)}(G)(n=\infty, 1,2, \cdots)$. Similarly we see that the left and the right translations on $\mathcal{E}_{n}(G)$ are topological in regard to any of $\tau_{r}, \tau_{l}$ and $\tau_{+}$.

Lemma 1.7. Let $N$ be a compact normal subgroup of $G$. For each $n=\infty, 1,2, \cdots$, the algebra isomorphism $g \mapsto g \circ \pi_{N}^{G}$ of $\mathcal{E}_{n}(G / N)$ onto $\mathcal{E}_{n}(G, N)$ (Corollary to Lemma 1.3) is also a homeomorphism relative to each of $\tau_{r}, \tau_{l}$ and $\tau_{+}$.

Proof. From (1.3) we have, for any $C \in \operatorname{Com}(G)$ and $\alpha_{1}, \cdots, \alpha_{p}, \beta_{1}, \cdots, \beta_{q}$ $\in R(G)(0 \leqq p+q<n+1)$,

$$
P_{C, \alpha_{1}, \cdots, \alpha_{p}}^{\beta_{1}, \cdots, \beta_{q}}\left(g \circ \pi_{N}^{G}\right)=P_{\pi_{N}^{G}(C), \bar{\alpha}_{1}, \cdots, \bar{\alpha}_{p}}^{\bar{\beta}_{1}, \ldots, \bar{\beta}_{q}}(g) \quad\left(g \in \mathcal{E}_{n}(G)\right),
$$

where $\bar{\alpha}_{i}=\bar{\pi}_{N}^{G}\left(\alpha_{i}\right), \bar{\beta}_{j}=\bar{\pi}_{N}^{G}\left(\beta_{j}\right)$. Hence, in view of $(0.10)$, the assertion follows. q. e. d.

Let $F$ be a closed subset of $G$ and $N$ a compact subgroup. We denote by $\mathscr{D}_{n}(G ; F)$ the linear subspace $\left\{f \in \mathscr{D}_{n}(G) ; \operatorname{supp}(f) \leqq F\right\}$ of $\mathscr{D}_{n}(G)(n=\infty, 1,2, \cdots)$, and put

$$
\begin{aligned}
& \mathscr{D}_{n}(G, N ; F)_{\rho}=\mathscr{D}_{n}(G, N)_{\rho} \cap \mathscr{D}_{n}(G ; F), \\
& \mathscr{D}_{n}(G, N ; f)_{\sigma}=\mathscr{D}_{n}(G, N)_{\sigma} \cap \mathscr{D}_{n}(G ; F), \\
& \mathscr{D}_{n}(G, N ; F)-\mathscr{D}_{n}(G, N) \cap \mathscr{D}_{n}(G ; F) .
\end{aligned}
$$

Note that these spaces are identical if $N$ is normal.

Lemma 1.8. Let $G_{1}$ be a pro-Lie open subgroup of $G$, and $(N, C) \in \boldsymbol{H}\left(G_{1}\right) \times$ Com $(G)$. Then $\tau_{r}, \tau_{l}$ and $\tau_{+}$induce the same topology on each of $\mathscr{D}_{n}(G, N ; C)_{\rho}$, $\mathscr{D}_{n}(G, N ; C)_{\sigma}$ and $\mathscr{D}_{n}(G, N ; C)(n=\infty, 1,2, \cdots)$, and make it a Fréchet space.

Proof. $\mathscr{D}_{n}^{(r)}(G, N ; C)_{\sigma}, \mathscr{D}_{n}^{(l)}(G, N ; C)_{\sigma}$ and $\mathscr{D}_{n}^{(+)}(G, N ; C)_{\sigma}$ are topologically 
isomorphic to $\mathscr{D}_{n}^{(l)}\left(G, N ; C^{-1}\right)_{\rho}, \mathscr{D}_{n}^{(r)}\left(G, N ; C^{-1}\right)_{\rho}$ and $\mathscr{D}_{n}^{(+)}\left(G, N ; C^{-1}\right)_{\rho}$ respectively under the map $f \mapsto \check{f}$. Besides, $\mathscr{D}_{n}(G, N ; C)$ is a $\tau_{+}$-closed subset of $\mathscr{D}_{n}(G, N ; C)_{\rho}$. Hence, for the proof of the lemma, it suffices to show that $\tau_{r}$, $\tau_{l}$ and $\tau_{+}$coincide with one another on $\mathscr{D}_{n}(G, N ; C)_{\rho}$ and make it a Fréchet space.

(I) First suppose that $G$ is Lie-projective and $G_{1}=G$. Then, from Lemma 1.7, $\mathscr{D}_{n}^{(+)}(G, N ; C N)$ is topologically isomorphic to $\mathscr{D}_{n}^{(+)}\left(G / N ; \pi_{N}^{G}(C)\right)$. Here, in view of finite dimensionality of $G / N$ and Lemma 1.4 , it is plain that $\mathscr{D}_{n}^{(+)}(G / N$; $\left.\pi_{N}^{G}(C)\right)$ is a Fréchet space. Hence so is $\mathscr{D}_{n}^{(+)}(G, N ; C N)$. Then its closed linear subspace $\mathscr{D}_{n}^{(+)}(G, N ; C)$ is also Fréchet. The same reasoning shows that $\mathscr{D}_{n}^{(r)}(G, N ; C)$ and $\mathscr{D}_{n}^{(l)}(G, N ; C)$ are also Fréchet spaces.

(II) Let $G$ and $G_{1}$ be arbitrary. Let $\left\{x_{1} G_{1}, \cdots, x_{s} G_{1}\right\}$ be the totality of the left $G_{1}$-cosets of $G$ meeting $C$. Put $C_{i}=C \cap x_{i} G_{1}(i=1, \cdots, s)$. Then, as is easily seen,

$$
\mathscr{D}_{n}^{(+)}(G, N ; C)_{\rho}=\sum_{i=1}^{s} \mathscr{D}_{n}^{(+)}\left(G, N ; C_{i}\right)_{\rho} \quad \text { (topological direct sum). }
$$

Here, since left translations on $\mathcal{E}_{n}^{(+)}(G)$ are topological, one sees that each $\mathscr{D}_{n}^{(+)}\left(G, N ; C_{i}\right)_{\rho}$ is topologically isomorphic to $\mathscr{D}_{n}^{(+)}\left(G_{1}, N ; x_{i}^{-1} C_{i}\right)$, which is a Fréchet space by (I). Thus it follows that $\mathscr{D}_{n}^{(+)}(G, N ; C)_{\rho}$ is a Fréchet space. By the same reasoning we see that $\mathscr{D}_{n}^{(r)}(G, N ; C)_{\rho}$ and $\mathscr{D}_{n}^{(l)}(G, N ; C)_{\rho}$ are also Fréchet spaces. Then the identity map of $\mathscr{D}_{n}^{(+)}(G, N ; C)_{\rho}$ onto each of $\mathscr{D}_{n}^{(r)}(G, N ; C)_{\rho}$ and $\mathscr{D}_{n}^{(l)}(G, N ; C)_{\rho}$, which is of course continuous, is topological by the open mapping theorem. The proof has thus been completed. q.e.d.

Let $G_{1}$ be any pro-Lie open subgroup of $G$. In view of Corollary 3 to Theorem 1.2 one has, for each $n=\infty, 1,2, \cdots$,

$$
\begin{aligned}
\mathscr{D}_{n}(G) & =\bigcup\left\{\mathscr{D}_{n}(G, N ; C)_{\rho} ;(N, C) \in \boldsymbol{H}\left(G_{1}\right) \times \operatorname{Com}(G)\right\} \\
& =\bigcup\left\{\mathscr{D}_{n}(G, N ; C)_{\sigma} ;(N, C) \in \boldsymbol{H}\left(G_{1}\right) \times \operatorname{Com}(G)\right\} \\
& =\bigcup\left\{\mathscr{D}_{n}(G, N ; C) ;(N, C) \in \boldsymbol{H}\left(G_{1}\right) \times \operatorname{Com}(G)\right\} .
\end{aligned}
$$

Now let us consider $\operatorname{Com}(G)$ as an upper directed set under inclusion, and $\boldsymbol{H}\left(G_{1}\right) \times \operatorname{Com}(G)$ as a product directed set. Then $\mathscr{D}_{n}(G)$ can be topologized in three ways so as to be the inductive limits of the families $\left\{\mathscr{D}_{n}^{(+)}(G, N ; C)_{\rho}\right.$; $\left.(N, C) \in \boldsymbol{H}\left(G_{1}\right) \times \operatorname{Com}(G)\right\}, \quad\left\{\mathscr{D}_{n}^{(+)}(G, N ; C)_{\sigma} ; \quad(N, C) \in \boldsymbol{H}\left(G_{1}\right) \times \operatorname{Com}(G)\right\} \quad$ and $\left\{\mathscr{D}_{n}^{(+)}(G, N ; C) ;(N, C) \in \boldsymbol{H}\left(G_{1}\right) \times \operatorname{Com}(G)\right\}$. Denote the topologies on $\mathscr{D}_{n}(G)$ thus defined by $\tau_{\rho}^{G_{1}}, \tau_{o}^{G_{1}}$ and $\tau^{G_{1}}$ respectively without regard to $n$.

Lemma 1.9. The topologies $\tau_{\rho}^{G_{1}}, \tau_{\sigma}^{G_{1}}$ and $\tau^{G_{1}}$ do not depend on the special choice of the pro-Lie open subgroup $G_{1}$ of $G$. Besides, they coincide with one another on each $\mathscr{D}_{n}(G)(n=\infty, 1,2, \cdots)$.

proof. The first half immediately follows from Lemma 0.16. Next, for any $(N, C) \in \boldsymbol{H}\left(G_{1}\right) \times \operatorname{Com}(G)$, the canonical injection of $\mathscr{D}_{n}^{(+)}(G, N ; C)$ into 
$\mathscr{D}_{n}^{(+)}(G, N ; C)_{\rho}$ is conntinuous. Hence $\tau^{G_{1}} \geqq \tau_{\rho}^{G_{1}}$. While, using Corollary 1 to Theorem 1.2, one sees that each sequence in $\mathscr{D}_{n}^{(+)}(G, N ; C)_{\rho}$ converging to 0 is a sequence in $\mathscr{D}_{n}^{(+)}\left(G, N^{\prime} ; C\right)$ converging to 0 for some $N^{\prime} \in \boldsymbol{H}\left(G_{1}\right)$. Hence $\boldsymbol{\tau}_{\rho}^{G_{1}}$ $\geqq \tau^{G_{1}}$. Therefore $\tau_{\rho}^{G_{1}}=\tau^{G_{1}}$. The coincidence of $\boldsymbol{\tau}_{\sigma}^{G_{1}}$ and $\boldsymbol{\tau}^{G_{1}}$ is shown similarly. q.e.d.

Definition 1.7. The same topologies $\tau_{\rho}^{G_{1}}, \tau_{\sigma}^{G_{1}}$ and $\tau^{G_{1}}$, which do not depend on the special choice of $G_{1}$, are denoted by $\tau_{*}$. Each space $\mathscr{D}_{n}(G)(n=\infty, 1$, $2, \cdots)$ equipped with $\tau_{*}$ is denoted by $\mathscr{D}_{n}^{(*)}(G)$. A linear subspace $\mathcal{K}$ of $\mathscr{D}_{n}(G)$ inheriting $\tau_{*}$ is also denoted by $\kappa^{(*)}$.

From definition, $\tau_{*}$ is finer than $\tau_{+}$relativized to $\mathscr{D}_{n}(G)$ and coincides with it on each of $\mathscr{D}_{n}(G, N ; C)_{\rho}$ and $\mathscr{D}_{n}(G, N ; C)_{\sigma}\left((N, C) \in \boldsymbol{H}\left(G_{1}\right) \times \operatorname{Com}(G)\right)$. As a (strict) inductive limit of Fréchet spaces, each $\mathscr{D}_{n}^{(*)}(G)$ is barrelled and bornological. If $G$ is finite-dimensional, $\mathscr{D}_{n}^{(*)}(G)$ reduces to the inductive limit of the family $\left\{\mathscr{D}_{n}^{(+)}(G ; C) ; C \in \operatorname{Com}(G)\right\}$. In particular, if $G$ is a Lie group, $\mathscr{D}_{\infty}^{(*)}(G)$ is the usual Schwartz space.

Remark 1.2. Let $G_{1}$ be a pro-Lie open subgroup of $G$. $\tau_{*}$ can be defined in two steps, too, i.e., the following two hold for each $n=\infty, 1,2, \cdots$.

(i) For each $N \in \boldsymbol{H}\left(G_{1}\right)$, the space $\mathscr{D}_{n}^{(*)}(G, N)_{\rho}$ is the inductive limit of the family $\left\{\mathscr{D}_{n}^{(+)}(G, N ; C)_{\rho} ; C \in \operatorname{Com}(G)\right\}$.

(ii) $\mathscr{D}_{n}^{(*)}(G)$ coincides with the inductive limit of $\left\{\mathscr{D}_{n}^{(*)}(G, N)_{\rho} ; N \in \boldsymbol{H}\left(G_{1}\right)\right\}$.

Proof. Let us check (i). Let $\tau_{N}$ be the inductive limit topology on $\mathscr{D}_{n}(G, N)$ defined by $\left\{\mathscr{D}_{n}^{(+)}(G, N ; C)_{\rho} ; C \in \operatorname{Com}(G)\right\}$. Then, plainly, $\tau_{N} \geqq \tau_{*}$ on $\mathscr{D}_{n}(G, N)_{\rho}$. While, $\rho_{N}$ maps $\mathscr{D}_{n}^{(*)}(G)$ onto $\mathscr{D}_{n}(G, N)_{\rho}$ with $\tau_{N}$ continuously because it maps each $\mathscr{D}_{n}\left(G, N^{\prime} ; C\right)_{\rho}\left(\left(N^{\prime}, C\right) \in \boldsymbol{H}\left(G_{1}\right) \times \operatorname{Com}(G)\right)$ into $\mathscr{D}_{n}(G, N ; C N)_{\rho} \boldsymbol{\tau}_{l}$-continuously (see 1.1). Since $\rho_{N}$ behaves as the identity map on $\mathscr{D}_{n}(G, N)_{\rho}$, this shows that $\tau_{*} \geqq \tau_{N}$ on $\mathscr{D}_{n}(G, N)_{\rho}$. q. e. d.

Remark 1.3. Let $G_{1}$ be a pro-Lie open subgroup of $G$, and $\left\{\Gamma_{\lambda}=x_{\lambda} G_{1}\right\}_{\lambda \in A}$ the totality of left $G_{1}$-cosets of $G$. Then, for each $n=\infty, 1,2, \cdots$, the following hold. (The employment of the right $G_{1}$-cosets gives the parallel assertions with obvious alterations in (i) and (ii).)

(i) Each $\mathscr{D}_{n}^{(*)}\left(G ; \Gamma_{\lambda}\right)$ is the inductive limit of the family $\left\{\mathscr{D}_{n}^{(+)}(G, N ; C)_{\rho}\right.$; $\left.(N, C) \in \boldsymbol{H}\left(G_{1}\right) \times \operatorname{Com}\left(\Gamma_{\lambda}\right)\right\}$.

(ii) $\mathscr{D}_{n}^{(*)}\left(G ; \Gamma_{\lambda}\right)$ is isomorphic to $\mathscr{D}_{n}^{(*)}\left(G ; G_{1}\right)$ under the map $f \mapsto_{x_{\lambda}} f$. And $\mathscr{D}_{n}^{(*)}\left(G ; G_{1}\right)$ is isomorphic to $\mathscr{D}_{n}^{(*)}\left(G_{1}\right)$ canonically.

(iii) $\mathscr{D}_{n}^{(*)}(G)=\sum_{\lambda \in \Lambda} \mathscr{D}_{n}^{(*)}\left(G ; \Gamma_{\lambda}\right)$ (topological direct sum).

Proof. Let $\tau_{\lambda}$ be the inductive limit topology on $\mathscr{D}_{n}\left(G ; \Gamma_{\lambda}\right)$ defined by $\left\{\mathscr{D}_{n}^{(+)}(G, N ; C)_{\rho} ;(N, C) \in \boldsymbol{H}\left(G_{1}\right) \times \operatorname{Com}\left(\Gamma_{\lambda}\right)\right\}$. Then, plainly, $\tau_{\lambda} \geqq \tau_{*}$ on $\mathscr{D}_{n}\left(G ; \Gamma_{\lambda}\right)$. While, for any $(N, C) \in \boldsymbol{H}\left(G_{1}\right) \times \operatorname{Com}(G)$, the defining function $\chi_{\lambda}$ of $\Gamma_{\lambda}$ belongs to $\mathcal{E}_{\infty}(G, N)_{\rho}$ and so the map $f \mapsto \chi_{\lambda} f$ carries $\mathscr{D}_{n}^{(+)}(G, N ; C)_{\rho}$ into $\mathscr{D}_{n}^{(+)}(G, N ; C$ 
$\left.\cap \Gamma_{\lambda}\right)_{\rho}$ continuously. Thus this map is continuous from $\mathscr{D}_{n}^{(*)}(G)$ onto $\mathscr{D}_{n}\left(G ; \Gamma_{\lambda}\right)$ with $\tau_{\lambda}$. Hence $\tau_{*} \geqq \tau_{\lambda}$ on $\mathscr{D}_{n}\left(G ; \Gamma_{\lambda}\right)$, which proves (i). (ii) is immediate from (i) and the continuity of left translations on $\mathscr{D}_{n}^{(+)}(G)$. The check of (iii) is left to the reader. q. e. d.

\subsection{Some properties of the spaces $\mathscr{D}_{n}^{(*)}(G)$.}

Proposition 1.1. Let $G_{1}$ be any pro-Lie open subgroup of $G$. Each bounded subset of $\mathscr{D}_{n}^{(*)}(G)(n=\infty, 1,2, \cdots)$ is included in $\mathscr{D}_{n}(G, N ; C)$ for some $(N, C) \in$ $\boldsymbol{H}\left(G_{1}\right) \times \operatorname{Com}(G)$.

Proof. Let $\mathscr{B}$ be a bounded subset of $\mathscr{D}_{n}^{(*)}(G)$. We can show in the same way as in the proof of [12], p. 74, Propositions 2 that $\mathscr{B} \subseteq \mathscr{D}_{n}(G ; C)$ for some $C \in \operatorname{Com}(G)$. On the other hand, since $\tau_{r}$ and $\tau_{l}$ are coarser than $\tau_{*}$ on $\mathscr{D}_{n}(G)$, $\mathscr{B}$ is bounded relative to each of them, too. Hence it satisfies the condition of

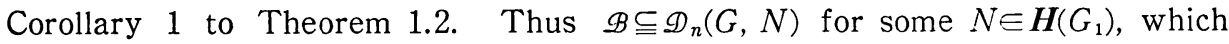
completes the proof. q.e.d.

Lemma 1.10. Let $N$ be a compact subgroup of $G$. $\rho_{n}$ induces a continuous projection on each $\mathscr{D}_{n}^{(*)}(G)(n=\infty, 1,2, \cdots)$.

Proof. Take a pro-Lie open subgroup $G_{1}$ of $G$. For each $\left(N^{\prime}, C\right) \in \boldsymbol{H}\left(G_{1}\right) \times$ Com $(G), \rho_{N}$ maps $\mathscr{D}_{n}^{(l)}\left(G, N^{\prime} ; C\right)_{\sigma}$ into $\mathscr{D}_{n}^{(l)}\left(G, N^{\prime} ; C N\right)_{\sigma}$ continuously (1.1). Hence the lemma. q.e.d.

Lemma 1.11. Let $N$ be a compact normal subgroup of $G$. For each $n=\infty$, $1,2, \cdots$, the map $g \in \mathscr{D}_{n}(G / N) \mapsto g \circ \pi_{N}^{G}$ sets up a topological linear isomorphism of $\mathscr{D}_{n}^{(*)}(G / N)$ onto $\mathscr{D}_{n}^{(*)}(G, N)$.

Proof. Denote this map by $\phi$. From Corollary to Lemma 1.3, $\phi$ is a linear isomorphism of $\mathscr{D}_{n}(G / N)$ onto $\mathscr{D}_{n}(G, N)$ in the algebraic sense. Take a pro-Lie open subgroup $G_{1}$ of $G$, and put $G_{1}^{\prime}=\pi_{N}^{G}\left(G_{1}\right)$. Then, plainly, $G_{1}^{\prime}$ is open and pro-Lie in $G / N$. Besides, there holds $\boldsymbol{H}\left(G_{1}^{\prime}\right)=\left\{\pi_{N}^{G}(K) ; K \in \boldsymbol{H}\left(G_{1}\right)\right\}$. Now take any $(K, C) \in \boldsymbol{H}\left(G_{1}\right) \times \operatorname{Com}(G)$. Then, by Lemma 1.7 , the spaces $\mathscr{D}_{n}^{(+)}\left(G / N, \pi_{N}^{G}(K)\right.$; $\left.\pi_{N}^{G}(C)\right)_{\rho}$ and $\mathscr{D}_{n}^{(+)}(G, K N ; C N)_{\rho}$ are isomorphic under $\psi$. Since the latter space is included in $\mathscr{D}_{n}^{(+)}(G, K ; C N)_{\rho}$, this shows in particular that $\dot{\phi}$ is continuous from $\mathscr{D}_{n}^{(*)}(G / N)$ into $\mathscr{D}_{n}^{(*)}(G, N)$. On the other hand, $\psi^{-1} \circ \rho_{N}$ maps $\mathscr{D}_{n}^{(+)}(G, K ; C)_{\rho}$ into $\mathscr{D}_{n}^{(+)}\left(G / N, \pi_{N}^{G}(K) ; \pi_{N}^{G}(C)\right)_{\rho}$ continuously because $\rho_{N}$ maps $\mathscr{D}_{n}^{(+)}(G, K ; C)_{\rho}$ into $\mathscr{D}_{n}^{(+)}(G, K N ; C N)_{\rho}$ continuously. Hence $\psi^{-1} \circ \rho_{N}$ is continuous from $\mathscr{D}_{n}^{(*)}(G)$ onto $\mathscr{D}_{n}^{(*)}(G / N)$. Therefore $\psi^{-1}$ is continuous from $\mathscr{D}_{1}^{(*)}(G, N)$ onto $\mathscr{D}_{n}^{(*)}(G / N)$, which completes the proof. q.e.d.

Lemma 1.12. Suppose $G$ is Lie-projective and put $\boldsymbol{H}=\left\{c(N) ; N \in \boldsymbol{H}_{0}(G)\right\}, a$ cofinal subfamily of $\boldsymbol{H}(G)$ (Corollary to Lemma 0.14). Then, for each $f \in \mathscr{D}_{n}(G)$ $(n=\infty, 1,2, \cdots)$, the net $\left\{\rho_{N}(f) ; N \in \boldsymbol{H}\right\}$ is bounded and converges to $f$ in $\mathscr{D}_{n}^{(*)}(G)$. 
Proof. Choose $\left(N_{0}, C\right) \in \boldsymbol{H}(G) \times \operatorname{Com}(G)$ so that $f \in \mathscr{D}_{n}(G, N ; C)$, and take the largest connected compact normal subgroup $L$ of $G$ ([8], Theorem 14). Then the net in question lies in $\mathscr{D}_{n}\left(G, N_{0} ; C L\right)$. It is obviously $\tau_{l}$-bounded and, by Lemma $1.2, \tau_{l}$-converges to $f$. Hence the lemma. q.e.d.

The next theorem can now be verified by following the proof of [5], Theorem 1.

Theorem 1.3. Each space $\mathscr{D}_{n}^{(*)}(G)(n=\infty, 1,2, \cdots)$ is complete.

Proof. Take an almost connected open subgroup $G_{1}$ of $G$. In view of Remark 1.3 and the fact that the topological direct sum of complete locally convex spaces is complete, it suffices to show that $\mathscr{D}_{n}^{(*)}\left(G_{1}\right)$ is complete. Therefore we can assume that $G$ itself is almost connected (hence $\sigma$-compact). Put $\mathscr{H}=\mathscr{D}_{n}^{(*)}(G)$ and denote by $\mathscr{H}^{\prime}$ its topological dual. Let $u$ be any linear form of $\mathscr{H}^{\prime}$ which is $\sigma\left(\mathscr{H}^{\prime}, \mathscr{H}\right)$-continuous on each equicontinuous subset of $\mathscr{H}^{\prime}$. The proof depends on Grothendieck's completion theorem ([4], Chap. 4, §3, exercise 3 ). Thus our task is to show that some element of $\mathscr{H}$ corresponds to $u$ canonically.

(I) For a compact subgroup $N$ of $G$, put $\mathscr{H}_{N}=\rho_{N}(\mathscr{H}), \mathcal{K}_{N}=\operatorname{ker} \rho_{N}, \mathscr{H}_{N}^{\prime}=$ $\rho_{N}^{\prime}\left(\mathscr{H}^{\prime}\right)$ and $\mathcal{K}_{N}^{\prime}=\operatorname{ker} \rho_{N}^{\prime}$, where $\rho_{N}^{\prime}$ denotes the adjoint of $\rho_{N}$ and $\mathscr{T}_{N}$ inherits $\tau_{*}$. Then, evidently, (i) $\mathcal{K}_{N}^{\prime}$ is the annihilator of $\mathscr{H}_{N}$ in $\mathscr{H}^{\prime}$; (ii) $\mathscr{H}^{\prime}=\mathscr{H}_{N}+\mathcal{K}_{N}$, $\mathscr{H}^{\prime}=\mathscr{H}_{N}^{\prime}+\mathcal{K}_{N}^{\prime}$ (direct sum); (iii) $\mathscr{H}_{N}^{\prime}$ can be viewed canonically as the topological dual of $\mathscr{H}_{N}$; (iv) if $N_{1} \cong N_{2}$, then $\mathscr{H}_{N_{1}}^{\prime} \supseteqq \mathcal{H}_{N_{2}}^{\prime}$ and $\mathcal{K}_{N_{1}}^{\prime} \cong \mathcal{K}_{N_{2}}^{\prime}$; (v) $\rho_{N_{1}}\left(\mathscr{H}_{N_{2}}\right) \subseteq \mathscr{H}_{N_{2}}$ for any $N_{1}, N_{2}$.

(II) Let $\boldsymbol{H}$ be as in Lemma 1.12. For each $T \in \mathcal{T}^{\prime}$, put $X_{T}=\left\{\rho_{N}^{\prime}(T) ; N \in \boldsymbol{H}\right\}$. Then, from Lemma 1.12 , its weak closure $\bar{X}_{T}$ is weakly bounded (hence equicontinuous because of the barrelledness of $\mathscr{H}$ ) and contains $T$.

(III) Put $X=\bigcup\left\{\mathscr{H}_{N}^{\prime} ; N \in \boldsymbol{H}\right\}$. Then there exists an $N_{u} \in \boldsymbol{H}$ s.t. $u(T)=0$ for all $T \in X \cap \mathcal{K}_{N_{u}}^{\prime}$. Indeed, if otherwise, (iv) of (I) enables us to choose a sequence $\left(N_{j}, T_{j}\right)(j=1,2,3, \cdots)$ so that $N_{j} \supseteqq N_{j+1}, T_{j} \in \mathscr{H}_{N_{j+1}}^{\prime} \cap \mathcal{K}_{N_{j}}^{\prime}$ and $u\left(T_{j}\right)=1$. Put $N_{\infty}=\bigcap_{j=1}^{\infty} N_{j}$. Then $N_{j} / N_{\infty} \in \boldsymbol{H}\left(G / N_{\infty}\right)$ and $\bigcap_{j=1}^{\infty} N_{j} / N_{\infty}=\left\{\pi_{N_{\infty}}^{G}(e)\right\}$. Since each $N_{j} / N_{\infty}$ is connected, this shows by Lemma 0.3 and Corollary to Lemma 0.14 that $\left\{N_{j} / N_{\infty} ; j=1,2,3, \cdots\right\}$ is cofinal in $\boldsymbol{H}\left(G / N_{\infty}\right)$. On the other hand, $G / N_{\infty}$ is $\sigma$-compact as well as $G$. Therefore it follows that $\mathscr{D}_{n}^{(*)}\left(G / N_{\infty}\right)$ is an $(L F)$ -

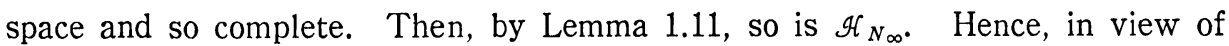
(iii) of (I), there exists an $f \in \mathscr{H}_{N_{\infty}}$ s.t. $u(T)=T(f)$ for all $T \in \mathscr{H}_{N_{\infty}}^{\prime}$. Since $\mathscr{H}_{N \infty}=\bigcup_{j=1}^{\infty} \mathscr{H}_{N_{j}}$ and so $f \in \mathscr{H}_{N_{j_{0}}}$ for some $j_{0}$, it follows that $u\left(T_{j_{0}}\right)=T_{j_{0}}(f)=0$ $\left(\because T_{j_{0}} \in \mathcal{K}_{N_{j_{0}}}^{\prime}\right)$, which is a contradiction.

(IV) Now suppose $T \in \mathcal{K}_{N_{u}}^{\prime}$. Then, by (i), (v) of (I), it is seen that $X_{T} \leqq$ $X \cap \mathcal{K}_{N_{u}}^{\prime}$. Hence $u=0$ on $X_{T}$ and so, by (II) and the assumption on $u, u(T)=0$. On the other hand, $\mathscr{H}_{N_{u}}$ is complete because $\mathscr{D}_{n}^{(*)}\left(G / N_{u}\right)$ is evidently an $(L F)$ space. Therefore there exists an $f_{u} \in \mathscr{H}_{N_{u}}$ s.t. $u(T)=T\left(f_{u}\right)$ for $T \in \mathscr{H}^{\prime}{ }_{N_{u}}$. Hence, after all, in view of (i), (ii) of (I), there holds $u(T)=T\left(f_{u}\right)$ for all $T \in \mathscr{T}^{\prime}$. This completes the proof. q.e.d. 
Lemma 1.13. Each of the left and the right translations and the inversion induces on $\mathscr{D}_{n}^{(*)}(G)$ a topological linear automorphism $(n=\infty, 1,2, \cdots)$.

Proof. Let $G_{1}$ be a pro-Lie open subgroup of $G$ and $(N, C) \in \boldsymbol{H}\left(G_{1}\right) \times \operatorname{Com}(G)$. The map $f \mapsto{ }_{x} f$ (resp. $\left.f \mapsto \check{f}\right)$ carries $\mathscr{D}_{n}^{(+)}(G, N ; C)_{\rho}$ into $\mathscr{D}_{n}^{(+)}\left(G, N ; x^{-1} C\right)_{\rho}$ (resp. $\left.\mathscr{D}_{n}^{(+)}\left(G, N ; C^{-1}\right)_{\sigma}\right)$ continuously (1.2). Hence the lemma. q.e.d.

Lemm 1.14. For each $h \in \mathcal{E}_{n}(G)$, the map $f \mapsto h f$ on $\mathscr{D}_{n}^{(*)}(G)$ is continuous $(n=\infty, 1,2, \cdots)$.

Proof. Let $G_{1}$ be a pro-Lie open subgroup of $G$ and $(N, C) \in \boldsymbol{H}\left(G_{1}\right) \times \operatorname{Com}(G)$. Let $f_{k}(k=1,2,3, \cdots)$ be any sequence in $\mathscr{D}_{n}^{(+)}(G, N ; C)$ converging to 0 . Then, using Corollary 1 to Theorem 1.2 , one sees that $h f_{k}$ is a sequence in $\mathscr{D}_{n}^{(+)}\left(G, N^{\prime} ; C\right)$ for some $N^{\prime} \in \boldsymbol{H}\left(G_{1}\right)$ coverging to 0 . Hence the lemma. q.e.d.

Lemma 1.15. For each $\varphi \in \mathscr{D}_{n}(G)(n=\infty, 1,2, \cdots)$, the maps $x \in G \mapsto{ }_{x} \varphi \in$ $\mathscr{D}_{n}^{(*)}(G)$ and $x \in G \mapsto \varphi_{x} \in \mathscr{D}_{n}^{(*)}(G)$ are continuous.

Proof. Let $G_{1}$ be a pro-Lie open subgroup of $G$. Choose $(N, C) \in \boldsymbol{H}\left(G_{1}\right) \times$ $\operatorname{Com}(G)$ so that $\varphi \in \mathscr{D}_{n}(G, N ; C)_{\rho}$. Then it is plain that the map $x \mapsto{ }_{x} \varphi$ is continuous from each relatively compact open subset $O$ of $G$ into $\mathscr{D}_{n}^{(r)}\left(G, N ; \bar{O}^{-1} C\right)_{\rho}$. Hence it is continuous from $G$ into $\mathscr{D}_{n}^{(*)}(G)$. q. e.d.

Since each $\mathscr{D}_{n}^{(*)}(G)$ is barrelled, one sees from Lemmas 1.13 and 1.15 that the left and the right regular representations of $G$ on $\mathscr{D}_{n}^{(*)}(G)$ are continuous (i. e., the maps $(x, \varphi) \rightarrow_{x^{-1}} \varphi$ and $(x, \varphi) \mapsto \varphi_{x}: G \times \mathscr{D}_{n}^{(*)}(G) \mapsto \mathscr{D}_{n}^{(*)}(G)$ are continuous). Lemma 1.14 shows in particular that each $\mathscr{D}_{n}^{(*)}(G)$ is a topological algebra (i. e., the multiplication in it is separately continuous).

\subsection{Projective topology for the spaces $\mathcal{E}_{n}(G)$.}

Definition 1.8. Without regard to $n(=\infty, 1,2, \cdots), \tau_{p}$ denotes the projective topology on $\mathcal{E}_{n}(G)$ defined by the linear maps $f \in \mathcal{E}_{n}(G) \mapsto \varphi f \in \mathscr{D}_{n}^{(*)}(G)$ $\left(\varphi \in \mathscr{D}_{n}(G)\right)$, i. e., the coarsest locally convex topology on $\mathcal{E}_{n}(G)$ which makes all these maps continuous. The space $\mathcal{E}_{n}(G)$ equipped with $\tau_{p}$ is denoted by $\mathcal{E}_{n}^{(p)}(G)$. Any linear subspace $\mathcal{K}$ of $\mathcal{E}_{n}(G)$ inheriting $\tau_{p}$ is also denoted by $\mathcal{K}^{(p)}$.

Lemma 1.16. (i) $\boldsymbol{\tau}_{p}$ is finer than $\boldsymbol{\tau}_{+}$. (ii) $\boldsymbol{\tau}_{p}$ is coarser than $\boldsymbol{\tau}_{*}$ on each $\mathscr{D}_{n}(G)$, but coincides with it on $\mathscr{D}_{n}(G ; C)$ for every $C \in \operatorname{Com}(G)$.

Proof. Note that for any $C \in \operatorname{Com}(G)$ one has $\varphi \in \mathscr{D}_{\infty}(G)$ s.t. $\varphi(x) \equiv 1$ on $C$ (In view of Remark 1.3 it suffices to see this for a Lie-projective $G$; and in such a case this is clear since, for $N \in \boldsymbol{H}_{0}(G)$, one has $\phi \in \mathscr{D}_{\infty}(G / N)$ s.t. $\phi\left(\pi_{N}^{G}(x)\right)$ $\equiv 1$ on $C$ ). (i): Let $f_{\nu}$ be any net in $\mathcal{E}_{n}^{(p)}(G)$ converging to 0 . For any $C \in$ $\operatorname{Com}(G)$ and $\alpha_{1}, \cdots, \alpha_{p}, \beta_{1}, \cdots, \beta_{q} \in R(G)(0 \leqq p+q<n+1)$, choose $\varphi \in \mathscr{D}_{\infty}(G)$ s.t. $\varphi(x) \equiv 1$ on a compact neighbourhood of $C$. Then $d_{\alpha_{1}}^{(r)} \cdots d_{\alpha_{p}}^{(r)} d_{\beta_{1}}^{(l)} \cdots d_{\beta_{q}}^{\phi_{2}} f_{\nu}=$ 
$d_{\alpha_{1}}^{(r)} \cdots d_{\alpha_{p}}^{(r)} d_{\beta_{1}}^{(r)} \cdots d_{\beta_{q}}^{(r)}\left(\varphi f_{\nu}\right)$ on $C$. By assumption $\varphi f_{\nu}$ converges to 0 in $\mathscr{D}_{n}(G)$ relative to $\tau_{*}$, hence a fortiori to $\tau_{+}$. Therefore

$$
P_{C, \cdots, \cdots, \alpha_{p}}^{\beta_{1}, \ldots, \beta_{q}}\left(f_{\nu}\right)=P_{C, \alpha_{1}, \cdots, \alpha_{p}}^{\beta_{1}, \ldots, \beta_{q}}\left(\varphi f_{\nu}\right) \longrightarrow 0 .
$$

Thus $f_{\nu} \rightarrow 0$ relative to $\boldsymbol{\tau}_{+}$, proving the assertion. (ii): The first half follows from Lemma 1.14. Now let $f_{\nu}$ be any net in $\mathscr{D}_{n}^{(p)}(G ; C)$ converging to 0 . Choose $\varphi \in \mathscr{D}_{\infty}(G)$ s.t. $\varphi(x) \equiv 1$ on $C$. Then $f_{\nu}=\varphi f_{\nu} \rightarrow 0$ relative to $\tau_{*}$. Hence $\boldsymbol{\tau}_{p} \geqq \boldsymbol{\tau}_{*}$ on $\mathscr{D}_{n}(G ; C)$, proving the second half. q. e. d.

Proposition 1.2. For a subset $\mathcal{B}$ of $\mathcal{E}_{n}(G)(n=\infty, 1,2, \cdots)$, the following conditions are equivalent: (a) $\boldsymbol{\tau}_{p}$-bounded; (b) $\boldsymbol{\tau}_{+}$-bounded; (c) $\boldsymbol{\tau}_{r}$-bounded; (d) $\boldsymbol{\tau}_{l^{-}}$ bounded. If $\mathscr{B} \subseteq \mathscr{D}_{n}(G ; C)$ for some $C \in \operatorname{Com}(G)$, these four are further equivalent to $\boldsymbol{\tau}_{*}$-boundedness.

Proof. Since $\boldsymbol{\tau}_{p} \geqq \boldsymbol{\tau}_{+} \geqq \boldsymbol{\tau}_{r}, \boldsymbol{\tau}_{l}$, the implication "(a) $\Rightarrow(\mathrm{b}) \Rightarrow(\mathrm{c})$ and (d)" is obvious. Now assume (c). Then, for any $\varphi \in \mathscr{D}_{n}(G), \varphi \mathscr{B}$ is $\tau_{r}$-bounded. Hence, by Theorem 1.2, it is a bounded subset of $\mathscr{D}_{n}^{(r)}(G, N \text {; } \operatorname{supp}(\varphi))_{\rho}$ for some $N \in$ $H\left(G_{1}\right)\left(G_{1}\right.$ being a pro-Lie open subgroup of $G$ ), and so, of $\mathscr{D}_{n}^{(*)}(G)$. This shows that $\mathscr{B}$ is $\tau_{p}$-bounded, i.e., that (a) holds. Analogously it is shown that (d) implies (a). The rest of the proposition is clear from Lemma 1.16, (ii). q.e.d.

Note. It is easily seen that if $G$ is finite-dimensional, $\boldsymbol{\tau}_{r}, \boldsymbol{\tau}_{l}, \boldsymbol{\tau}_{+}$and $\tau_{p}$ are identical.

Proposition 1.3. Each space $\mathcal{E}_{n}^{(p)}(G)(n=\infty, 1,2, \cdots)$ is complete.

Proof. Recall that $\mathscr{D}_{n}^{(*)}(G)$ and $\mathcal{E}_{n}^{(+)}(G)$ are complete. Take any Cauchy net $f_{\nu}$ in $\mathcal{E}_{n}^{(p)}(G)$ and $\varphi \in \mathscr{D}_{n}(G)$. Then the net $\varphi f_{\nu}$ is Cauchy in $\mathscr{D}_{n}^{(*)}(G)$ and so converges to some $\phi$ in $\mathscr{D}_{n}^{(*)}(G)$. While, since $\boldsymbol{\tau}_{+} \leqq \boldsymbol{\tau}_{p}$, the net $f_{\nu}$ is Cauchy also in $\mathcal{E}_{n}^{(+)}(G)$, and so converges to some $f$ in $\mathcal{E}_{n}^{(+)}(G)$. Then $\varphi f_{\nu} \rightarrow \varphi f$ in $\mathscr{D}_{n}^{(+)}(G)$. Since $\tau_{*} \geqq \tau_{+}$on $\mathscr{D}_{n}(G)$, we have thus $\phi=\varphi f$. Therefore $\varphi f_{\nu} \rightarrow \varphi f$ in $\mathscr{D}_{n}^{(*)}(G)$. Hence $f_{\nu} \rightarrow f$ in $\mathcal{E}_{n}^{(p)}(G)$, completing the proof. q. e.d.

One sees easily from Lemma 1.13 that each of the left and the right translations and the inversion on $\mathcal{E}_{n}^{(p)}(G)(n=\infty, 1,2, \cdots)$ is a topological linear automorphism, and from Lemma 1.14 that each $\mathcal{E}_{n}^{(p)}(G)$ is a topological algebra.

Remark 1.4. Let $G_{1}$ and $\left\{\Gamma_{\lambda}=x_{\lambda} G_{1}\right\}_{\lambda \in \Lambda}$ be as in Remark 1.3. For each $\lambda \in \Lambda$, put

$$
\mathcal{E}_{n}\left(G ; \Gamma_{\lambda}\right)=\left\{f \in \mathcal{E}_{n}(G) ; \operatorname{supp}(f) \subseteq \Gamma_{\lambda}\right\} \quad(n=\infty, 1,2, \cdots) .
$$

The following are easily checked.

(i) Each space $\mathcal{E}_{n}^{(p)}\left(G ; \Gamma_{\lambda}\right)$ is isomorphic to $\mathcal{E}_{n}^{(p)}\left(G ; G_{1}\right)$ under the map $f \mapsto_{x_{\lambda}} f$. Here $\mathcal{E}_{n}^{(p)}\left(G ; G_{1}\right)$ is isomorphic to $\mathcal{E}_{n}^{(p)}\left(G_{1}\right)$ canonically.

(ii) $\mathcal{E}_{n}^{(p)}(G)=\prod_{\lambda \in A} \mathcal{E}_{n}^{(p)}\left(G ; \Gamma_{\lambda}\right)$ (topological direct product). 
The next simple lemma is frequently used later on.

Lemma 1.17. Let $G_{1}$ be a pro-Lie open subgroup of $G$, and $N \in \boldsymbol{H}\left(G_{1}\right)$. If $f \in \mathcal{E}_{n}(G, N)_{\rho}(n=\infty, 2,3, \cdots)$, then $d_{\alpha}^{(r)} f \in \mathcal{E}_{n-1}(G, N)_{\rho}$ for all $\alpha \in R(G)$.

Proof. Let $\left\{\Gamma_{\lambda}=x_{\lambda} G_{1}\right\}_{\lambda \in \Lambda}$ be as in Remark 1.4. For each $\lambda \in \Lambda$, let $f_{\lambda}$ denote the $\mathcal{E}_{n}\left(G ; \Gamma_{\lambda}\right.$ )-component of $f$ (Remark 1.4, (ii)). Then, evidently, $f_{\lambda} \in$ $\mathcal{E}_{n}\left(G, N ; \Gamma_{\lambda}\right)_{\rho}$, where $\mathcal{E}_{n}\left(G, N ; \Gamma_{\lambda}\right)_{\rho}=\mathcal{E}_{n}\left(G ; \Gamma_{\lambda}\right) \cap \mathcal{E}_{n}(G, N)_{\rho}$. Put $h_{\lambda}={ }_{x_{\lambda}} f_{\lambda}$. Then $h_{\lambda} \in \mathcal{E}_{n}\left(G, N ; G_{1}\right)_{\rho}$. Here $\mathcal{E}_{n}\left(G, N ; G_{1}\right)_{\rho}=\mathcal{E}_{n}\left(G, N ; G_{1}\right)_{\sigma}$ since $N$ is normal in $G_{1}$. Thus, obviously, $d_{\alpha}^{(r)} h_{\lambda} \in \mathcal{E}_{n-1}\left(G, N ; G_{1}\right)_{\rho}$ for all $\alpha \in R(G)$. Then, by Lemma 1.1, (i), $d_{\alpha}^{(r)} f_{\lambda}=d_{\alpha}^{(r)}\left(x_{\lambda^{-1}} h_{\lambda}\right)={ }_{x_{\lambda}-1}\left(d_{\alpha}^{(r)} h_{\lambda}\right) \in \mathcal{E}_{n-1}\left(G, N ; \Gamma_{\lambda}\right)_{\rho}$. Hence the assertion. q.e.d.

1.5. Connections with the Bruhat spaces $\mathscr{D}(G)$ and $\mathcal{E}(G)$. In this section we clarify the connections of the spaces $\mathscr{D}_{\infty}^{(*)}(G)$ and $\mathcal{E}_{\infty}^{(p)}(G)$ with the Bruhat spaces $\mathscr{D}(G)$ and $\mathcal{E}(G)$, and also of the topological dual of the former spaces with those of the latter spaces.

Let $G_{1}$ be a pro-Lie open subgroup of $G$. Using the directed set $\boldsymbol{H}_{0}\left(G_{1}\right) \times$ Com $(G)$ instead of $\boldsymbol{H}\left(G_{1}\right) \times \operatorname{Com}(G)$, consider the inductive limit space $\mathscr{D}(G)_{\rho}$ (resp. $\left.\mathscr{D}(G)_{\sigma}\right)$ of the family $\left\{\mathscr{D}_{\infty}^{(+)}(G, N ; C)_{\rho} ;(N, C) \in \boldsymbol{H}_{0}\left(G_{1}\right) \times \operatorname{Com}(G)\right\}$ (resp. $\left.\left\{\mathscr{D}_{\infty}^{(+)}(G, N ; C)_{\sigma} ;(N, C) \in \boldsymbol{H}_{0}\left(G_{1}\right) \times \operatorname{Com}(G)\right\}\right)$. These two do not depend on the special choice of $G_{1}$ because of Lemma 0.16 , and are plainly identical if $G$ is Lie-projective. Here we can check the following two Remarks easily analogously with Remarks 1.2 and 1.3.

Remark 1.5. Let $G$ be Lie-projective. (i) For each $N \in \boldsymbol{H}_{0}(G)$, the topological linear subspace $\mathscr{D}_{\infty}(G, N)$ of $\mathscr{D}(G)_{\rho}\left(=\mathscr{D}(G)_{\sigma}\right)$ coincides with the inductive limit of $\left\{\mathscr{D}_{\infty}^{(+)}(G, N ; C) ; C \in \operatorname{Com}(G)\right\}$, hence with $\mathscr{D}_{\infty}^{(*)}(G, N)$ (by Remark 1.2, (i)). (ii) $\mathscr{D}(G)_{\rho}\left(=\mathscr{D}(G)_{\sigma}\right)$ coincides with the inductive limit of $\left\{\mathscr{D}_{\infty}^{(*)}(G, N)\right.$; $\left.N \in \boldsymbol{H}_{0}(G)\right\}$.

Remark 1.6. Let $G_{1}$ and $\left\{\Gamma_{\lambda}=x_{\lambda} G_{1}\right\}_{\lambda \in A}$ be as in Remark 1.3. For each $\lambda$ let $D\left(G ; \Gamma_{\lambda}\right)$ denote the topological linear subspace $\left\{f \in \mathscr{D}(G)_{\rho} ; \operatorname{supp}(f) \subseteq \Gamma_{\lambda}\right\}$ of $\mathscr{D}(G)_{\rho}$. Then,

(i) $\mathscr{D}\left(G ; \Gamma_{\lambda}\right)$ coincides with the inductive limit of $\left\{\mathscr{D}_{\infty}^{(+)}(G, N ; C)_{\rho} ;(N, C)\right.$ $\left.\in \boldsymbol{H}_{0}(G) \times \operatorname{Com}\left(\Gamma_{\lambda}\right)\right\}$.

(ii) $\mathscr{D}\left(G ; \Gamma_{\lambda}\right)$ is isomorphic to $\mathscr{D}\left(G ; G_{1}\right)$ under the map $f \mapsto_{x_{\lambda}} f$. And $\mathscr{D}\left(G ; G_{1}\right)$ is isomorphic to $\mathscr{D}\left(G_{1}\right)_{\rho}\left(=\mathscr{D}\left(G_{1}\right)_{\sigma}\right)$ canonically,

(iii) $\mathscr{D}(G)_{\rho}=\sum_{\lambda \in \Lambda} \mathscr{D}\left(G ; \Gamma_{\lambda}\right)$ (topological direct sum).

The employment of the right $G_{1}$-cosets enables us to obtain the parallel assertions with respect to $\mathscr{D}(G)_{\sigma}$.

In view of the definition of $\mathscr{D}(G)$ ([5], Definition 1 and Proposition 4), we see by Lemma 1.11, Remark 1.5, (ii) and Remark 1.6, (ii), (iii) that no matter $G$ is Lie-projective or not, $\mathscr{D}(G)_{\rho}$ and $\mathscr{D}(G)_{\sigma}$ are the same and nothing but $\mathscr{D}(G)$ 
including the topology: $\mathscr{D}(G)_{\rho}=\mathscr{D}(G)_{\sigma}=\mathscr{D}(G)$. This obviously yields $\mathscr{D}(G)=$ $\cup\left\{\mathscr{D}_{\infty}(G, N) ; N \in \boldsymbol{H}_{0}(G)\right\}\left(\cong \mathscr{D}_{\infty}(G)\right)$ as set. If $G$ is locally connected, $\mathscr{D}(G)=$ $\mathscr{D}_{\infty}^{(*)}(G)$ holds since in this case $\boldsymbol{H}_{0}\left(G_{1}\right)=\boldsymbol{H}\left(G_{1}\right)$ (Lemma 0.15 ).

Lemma 1.18. Let $C \in \operatorname{Com}(G)$, and $\left\{O_{1}, \cdots, O_{s}\right\}$ be an open covering of $C$. Then there exist functions $f_{1}, \cdots, f_{s}$ in $\mathscr{D}(G)^{+}$such that $\operatorname{supp}\left(f_{i}\right) \leqq O_{i}(i=1, \cdots, s)$ and $\sum_{i=1}^{s} f_{i}(x) \equiv 1$ on $C$.

Proof. [5], Proposition 2 gives this assertion for the Lie-projective $G$. The generalization to any $G$ is immediate in view of Remark 1.6, (ii). q. e.d.

The next simple lemma is used in 2.1.

Lemma 1.19. There exists a locally finite family $\left\{a_{\kappa}\right\}_{\kappa \in K}$ of functions in $\mathscr{D}(G)^{+}$(i.e., each compact subset of $G$ meets at most a finite number of the sets $\left.\operatorname{supp}\left(a_{\kappa}\right)\right)$ s.t. $\sum_{\kappa \in K} a_{\kappa}(x)=1$ for all $x \in G$.

Proof. Remark 1.6 allows us to assume that $G$ is Lie-projective. Take an $N \in \boldsymbol{H}_{0}(G)$. Then, since $G / N$ is a Lie group, there exists a locally finite family $\left\{g_{\kappa}\right\}_{\kappa \in K}$ of functions in $\mathscr{D}(G / N)$ s.t. $\sum_{\kappa \in K} g_{\kappa}\left(\pi_{N}^{G}(x)\right)=1 \quad(x \in G)$. So we have only to put $a_{\kappa}=g_{\kappa} \circ \pi_{N}^{G}(\kappa \in K)$. q.e. d.

By definitition $\mathcal{E}(G)$ consists of all $C$-valued functions $f$ on $G$ with the property that for each $x \in G$, there exist a neighbourhood $U$ of $x$ and $\varphi \in \mathscr{D}(G)$ s.t. $f=\varphi$ on $U$. In view of Lemma 1.18, this property of $f$ is equivalent to that $\varphi f \in \mathscr{D}(G)$ for all $\varphi \in \mathscr{D}(G)$. One has obviously $\mathcal{E}(G) \leqq \mathcal{E}_{\infty}(G)$ and $\mathscr{D}(G)=$ $\{f \in \mathcal{E}(G)$; $\operatorname{supp}(f)$ is compact $\} . \mathcal{E}(G)$ is equipped with the projective topology defined by the linear maps $f \in \mathcal{E}(G) \mapsto \varphi f \in \mathscr{D}(G)(\varphi \in \mathscr{D}(G))$. So, if $G$ is locally connected, $\mathcal{E}(G)=\mathcal{E}_{\infty}^{(p)}(G)$ holds since in this case $\mathscr{D}(G)=\mathscr{D}_{\infty}^{(*)}(G)$.

The following facts on $\mathscr{D}(G)$ and $\mathcal{E}(G)$ are well known ([5]) and used in the sequel: Lemmas $1.13,1.14$ and 1.15 with $\mathscr{D}(G), \mathcal{E}(G)$ in place of $\mathscr{D}_{\infty}^{(*)}(G)$, $\mathcal{E}_{\infty}^{(p)}(G)$ (resp.) are valid. $\mathscr{D}(G)$ and $\mathcal{E}(G)$ are topological algebras. The topology of $\mathscr{D}(G)$ is finer than that of $\mathcal{E}(G)$ relativized to it. $\mathscr{D}(G)$ is barrelled and bornological, and the left and the right regular representations of $G$ on it is continuous. Each bounded subset of $\mathscr{D}(G)$ lies in $\mathscr{D}_{\infty}(G, N ; C)$ for some $(N, C)$ $\boldsymbol{H}_{0}\left(G_{1}\right) \times \operatorname{Com}(G), G_{1}$ being any pro-Lie open subgroup of $G$. Each of $\mathscr{D}(G)$ and $\mathcal{E}(G)$ is a complete Montel space.

Furthermore there obtains the following

Remark 1.7. Let $G_{1}$ and $\left\{\Gamma_{\lambda}=x_{\lambda} G_{1}\right\}_{\lambda \in A}$ be as in Remark 1.6. For each $\lambda$ let $\mathcal{E}\left(G ; \Gamma_{\lambda}\right)$ denote the topological linear subspace $\left\{f \in \mathcal{E}(G)\right.$; $\left.\operatorname{supp}(f) \subseteq \Gamma_{\lambda}\right\}$ of $\mathcal{E}(G)$. Then,

(i) Each $\mathcal{E}\left(G ; \Gamma_{\lambda}\right)$ is isomorphic to $\mathcal{E}\left(G ; G_{1}\right)$ under the map $f_{\mapsto} f$. Here $\mathcal{E}\left(G ; G_{1}\right)$ is isomorphic to $\mathcal{E}\left(G_{1}\right)$ cononically.

(ii) $\mathcal{E}(G)=\prod_{\lambda \in \Lambda} \mathcal{E}\left(G ; \Gamma_{\lambda}\right)$ (topological direct product). 
Lemma 1.20. The topology of $\mathscr{D}(G)$ (resp. $\mathcal{E}(G)$ ) is finer than $\boldsymbol{\tau}_{*}$ (resp. $\left.\boldsymbol{\tau}_{p}\right)$ relativized there.

Proof. From construction of $\mathscr{D}(G)$ it is plain that the topology of $\mathscr{D}(G)$ is finer than $\tau_{*}$ relativized. Now let $f_{\nu}$ be any net in $\mathcal{E}(G)$ converging to 0 , and $\varphi \in \mathscr{D}_{\infty}(G)$. Take $\phi \in \mathscr{D}(G)$ s.t. $\phi(x) \equiv 1$ on $\operatorname{supp}(\varphi)$ (Lemma 1.18). Then $\varphi f_{\nu}$ $\left(=\varphi\left(\psi f_{\nu}\right)\right) \rightarrow 0$ in $\mathscr{D}_{\infty}^{(*)}(G)$ since $\phi f_{\nu} \rightarrow 0$ in $\mathscr{D}(G)$ and so in $\mathscr{D}_{\infty}^{(*)}(G)$. Hence $f_{\nu} \rightarrow 0$ in $\mathcal{E}_{\infty}^{(p)}(G)$, proving the other assertion. q.e.d.

Lemma 1.21. $\mathscr{D}(G)$ is dense in each of $\mathcal{E}(G), \mathscr{D}_{n}^{(*)}(G)$ and $\mathcal{E}_{n}^{(p)}(G) \quad(n=$ $\infty, 1,2, \cdots)$.

Proof. For each $C \in \operatorname{Com}(G)$, choose $\varphi_{C} \in \mathscr{D}(G)$ s.t. $\varphi_{C}(x) \equiv 1$ on $C$. Then, for any $f \in \mathcal{E}(G)$ and $\phi \in \mathscr{D}(G)$, the net $\left\{\psi\left(\varphi_{C} f\right) ; C \in \operatorname{Com}(G)\right\}$ converges to $\phi f$ in $\mathscr{D}(G)$. Hence $\left\{\varphi_{C} f ; C \in \operatorname{Com}(G)\right\}$ converges to $f$ in $\mathcal{E}(G)$. Thus $\mathscr{D}(G)$ is dense in $\mathcal{E}(G)$. Analogously it is shown that $\mathscr{D}_{n}(G)$ is dense in $\mathcal{E}_{n}^{(p)}(G)(n=$ $\infty, 1,2, \cdots)$. So it now suffices to show that $\mathscr{D}(G)$ is dense in each $\mathscr{D}_{n}^{(*)}(G)$. Take a pro-Lie open subgroup $G_{1}$ of $G$. For any $\varphi \in \mathscr{D}_{n}(G)$, choose $\left(N_{0}, C\right) \in$ $\boldsymbol{H}\left(G_{1}\right) \times \operatorname{Com}(G)$ so that $\varphi \in \mathscr{D}_{n}\left(G, N_{0} ; C\right)_{\sigma}$. Then the net $\left\{\rho_{N}(\varphi) ; N \in \boldsymbol{H}\right\}$ converges to $\varphi$ in $\mathscr{D}_{n}^{(l)}\left(G, N_{0} ; C N_{1}\right)_{\sigma}$ (Lemma 1.2), where $\boldsymbol{H}=\left\{N \in \boldsymbol{H}_{0}(G) ; N \subseteq N_{1}\right\}$ with a fixed $N_{1} \in \boldsymbol{H}_{0}\left(G_{1}\right)$. Since $\rho_{N}(\varphi) \in \mathscr{D}(G)(N \in \boldsymbol{H})$, the proof is complete. q.e.d.

Lemma 1.22. Suppose $G$ is Lie-projective and finite-dimensional. For each totally disconnected $N \in \boldsymbol{H}_{0}(G)$ (see Lemma 0.13), there exists a continuous linear map $g \mapsto \dot{g}$ of $\mathcal{E}_{0}(N)$ into $\mathscr{D}_{\infty}^{(*)}(G)$ such that (a) $\dot{g}=g$ on $N$ for all $g \in \mathcal{E}_{0}(N)$, and (b) $g \in \mathscr{D}(N) \Leftrightarrow \dot{g} \in \mathscr{D}(G)$.

Proof. By Lemma 0.11 there exists a subset $L$ of $G$ such that (i) $U=L N$ is an open neighbourhood of $e$ in $G$; (ii) the map $(y, z) \in L \times N \mapsto y z \in U$ is a homeomorphism; (iii) the map $\xi:\left(\pi_{N}^{G}(y), z\right) \in \pi_{N}^{G}(L) \times N \mapsto y z \in U$ is a local isomorphism of $(G / N) \times N$ into $G$. Choose relatively compact open subsets $V, W$ of $G$ so that $N \subseteq V, \bar{V} \leqq W$ and $\bar{W} \leqq U$. And choose $a \in \mathscr{D}(G)$ such that $a(x) \equiv 1$ on $V$ and $\operatorname{supp}(a) \subseteq W$ (Lemma 1.18). For each $g \in \mathcal{E}_{0}(N)$, denote by $g^{\prime}$ the trivial extension of $g$ to $\pi_{N}^{G}(L) \times N$ and regard it as a function on $U$ through $\xi$. We define

$$
\dot{g}(x)= \begin{cases}a(x) g^{\prime}(x) & \text { if } x \in U, \\ 0 & \text { otherwise (i. e., } x \in G \backslash U) .\end{cases}
$$

Then $\dot{g} \in \mathcal{E}_{0}(G)$, supp $(\dot{g}) \subseteq \bar{W}$ and $\dot{g}=g$ on $N$. Besides, for any $\alpha_{1}, \cdots, \alpha_{p} \in R(G)$ $(p=1,2,3, \cdots)$, we have

$$
d_{\alpha_{1}}^{(l)} \cdots d_{\alpha_{p}}^{(l)} \dot{g}(x)=\left(d_{\alpha_{1}}^{(l)} \cdots d_{\alpha_{p}}^{(l)} a\right)(x) g^{\prime}(x) \quad(x \in U) .
$$

Hence $\dot{g} \in \mathscr{D}_{\infty}(G ; \bar{W})$. Now let $g_{k}(k=1,2,3, \cdots)$ be any sequence in $\mathcal{E}_{0}(N)$ converging to 0 . Then, by (1.4) and construction of $g^{\prime}$, the sequence $\dot{g}_{k}$ converges 
to 0 in $D_{\infty}^{(l)}(G ; \bar{W})$. Hence, in view of Theorem 1.2 , it converges to 0 in $\mathscr{D}_{\infty}^{(l)}\left(G, N_{0} ; \bar{W}\right)_{\sigma}$ for some $N_{0} \in \boldsymbol{H}(G)$. This proves that the map $g \in \mathcal{E}_{0}(N) \mapsto$ $\dot{g} \in \mathscr{D}_{\infty}^{(*)}(G)$ is continuous. The linearity of this map is obvious. Now suppose $g \in \mathscr{D}(N)$. Then, since $\left\{N \cap N^{\prime} ; N^{\prime} \in \boldsymbol{H}_{0}(G)\right\}$ is cofinal in $\boldsymbol{H}_{0}(N)$ (Lemma 0.3 ), $g$ belongs to $\mathscr{D}_{\infty}\left(N, N \cap N_{1}\right)$ for some $N_{1} \in \boldsymbol{H}_{0}(G)$. So $g^{\prime}$ is constant on each $N \cap N_{1}$ coset of $G$ contained in $U$. Therefore, if we take $N_{2} \in \boldsymbol{H}_{0}(G)$ s.t. $a \in \mathscr{D}_{\infty}\left(G, N_{2}\right)$, then $\dot{g} \in \mathscr{D}_{\infty}\left(G, N \cap N_{1} \cap N_{2}\right) \subseteq \mathscr{D}(G)$. Conversely suppose that $\dot{g} \in \mathscr{D}(G)$, i. e., that $\dot{g} \in \mathscr{D}_{\infty}\left(G, N^{\prime}\right)$ for some $N^{\prime} \in \boldsymbol{H}_{0}(G)$. Then $g=\left.\dot{g}\right|_{N} \in \mathscr{D}_{\infty}\left(N, N \cap N^{\prime}\right) \subseteq \mathscr{D}(N)$. This completes the proof. q.e.d.

Now we prove the following

Proposition 1.4. Suppose $G$ is not locally connected. Then, (i) $\mathscr{D}(G) \subseteq \mathscr{D}_{\infty}(G){ }^{(4)}$ (ii) The topology of $D(G)$ is strictly finer than $\tau_{*}$ relativized there. (iii) $\mathcal{E}(G) \subseteq$ $\mathcal{E}_{\infty}(G)$. (iv) The topology of $\mathcal{E}(G)$ is strictly finer than $\tau_{p}$ relativized there.

Proof. (iii) is obviously implied by (i). Since $\mathscr{D}(G)$ (resp. $\mathcal{E}(G)$ ) is complete and dense in $\mathscr{D}_{\infty}^{(*)}(G)$ (resp. $\mathcal{E}_{\infty}^{(p)}(G)$ ), (ii) (resp. (iv)) is implied by (i) (resp. (iii)). Therefore we have only to prove (i). Here, in view of Remarks 1.3 and 1.6, it suffices to consider the case $G$ is Lie-projective. (I) First let $G$ be finitedimensional. Then $G$ is locally isomorphic to $(G / N) \times N$ for a totally disconnected $N \in \boldsymbol{H}_{0}(G)$ (Lemmas 0.11 and 0.13 ). Since $N$ is compact and not locally connected as well as $G$, we have a function $g \in \mathscr{D}_{\infty}(N) \backslash \mathscr{D}(N)$ by [6], Proposition 3.2. By Lemma $1.22 \mathrm{~g}$ extends to a function $\dot{g} \in \mathscr{D}_{\infty}(G) \backslash \mathscr{D}(G)$. (II) Next let $G$ be infinite-dimensional. In view of Lemma 0.15 we can take an $N^{\prime} \in$ $\boldsymbol{H}(G) \backslash \boldsymbol{H}_{0}(G)$. Since $G / N^{\prime}$ is finite-dimensional, Lie-projective and not locally connected, we have an $h \in \mathscr{D}_{\infty}\left(G / N^{\prime}\right) \backslash \mathscr{D}\left(G / N^{\prime}\right)$ by (I). Put $f=h \circ \pi_{N^{\prime}}^{G}$. Then it is easy to check that $f \in \mathscr{D}_{\infty}(G) \backslash \mathscr{D}(G)$. $\quad$ q. e. d.

Corollary. An almost connected $G$ is locally connected if and only if $\boldsymbol{H}_{1}(G)$ $=\boldsymbol{H}_{0}(G)$.

Proof. Obvious from Lemma 0.15, Corollary 3 to Theorem 1.2 and Proposition 1.4. q.e.d.

Next we consider the topological dual of the spaces $\mathscr{D}_{\infty}^{(*)}(G)$ and $\mathcal{E}_{\infty}^{(p)}(G)$ in connection with those of $\mathscr{D}(G)$ and $\mathcal{E}(G)$.

Definition 1.9. $\mathscr{D}^{\prime}(G), \mathscr{D}_{\infty}^{\prime}(G), \mathcal{E}^{\prime}(G)$ and $\mathcal{E}_{\infty}^{\prime}(G)$ denote the topological dual of the spaces $\mathscr{D}(G), \mathscr{D}_{\infty}^{(*)}(G), \mathcal{E}(G)$ and $\mathcal{E}_{\infty}^{(p)}(G)$ respectively. They are equipped with the strong topologies of dual, denoted by $\beta\left(\mathscr{D}^{\prime}, \mathscr{D}\right), \beta\left(\mathscr{D}_{\infty}^{\prime}, \mathscr{D}_{\infty}\right), \beta\left(\mathcal{E}^{\prime}, \mathcal{E}\right)$ and $\beta\left(\mathcal{E}_{\infty}^{\prime}, \mathcal{E}_{\infty}\right)$ (resp.).

By distributions on $G$ we mean the elements of $\mathscr{D}^{\prime}(G)$, i. e., the distributions

(4) Cf. [3], Theorem 2.2.2.8. 
in Bruhat's sense. As we know $([5]), \mathcal{E}^{\prime}(G)$ can be viewed algebraically as the linear subspace of $\mathscr{D}^{\prime}(G)$ consisting of all elements with compact support. Here the support of $T \in \mathscr{D}^{\prime}(G)$ means the smallest closed subset $F$ of $G$ s.t. $\langle\varphi, T\rangle=0$ for all $\varphi \in \mathscr{D}(G)$ with $\operatorname{supp}(\varphi) \cap F=\varnothing$. In view of Lemmas 1.20 and $1.21, \mathscr{D}_{\infty}^{\prime}(G)$ and $\mathcal{E}_{\infty}^{\prime}(G)$ can also be viewed algebraically as linear subspaces of $\mathscr{D}^{\prime}(G)$ in the canonical way. Under this convention, which we keep throughout, the following schema is evident:

$$
\begin{array}{ccc}
\mathcal{E}^{\prime}(G) & \subseteq \mathscr{D}^{\prime}(G) \\
U \| & \text { U\| } \\
\mathcal{E}_{\infty}^{\prime}(G) \subseteq & \mathscr{D}_{\infty}^{\prime}(G) .
\end{array}
$$

Here notice that if $A, B$ are two spaces appearing in (1.5) s.t. $A \subseteq B$, the topology of $A$ is finer than that of $B$ relativized to $A$.

Now suppose $T \in \mathcal{E}^{\prime}(G) \cap \mathscr{D}_{\infty}^{\prime}(G)$, and choose $a \in \mathscr{D}(G)$ s.t. $a(x) \equiv 1$ on a neighbourhood of $\operatorname{supp}(T)$, a compact set. Then $\langle\varphi, T\rangle=\langle a \varphi, T\rangle$ for $\varphi \in \mathscr{D}(G)$. Since $T \in \mathscr{D}_{\infty}^{\prime}(G)$, this implies that $T$ is $\tau_{p}$-continuous on $\mathscr{D}(G)$, i.e., that $T \in$ $\mathcal{E}_{\infty}^{\prime}(G)$. Hence $\mathcal{E}^{\prime}(G) \cap \mathscr{D}_{\infty}^{\prime}(G) \cong \mathcal{E}_{\infty}^{\prime}(G)$. Thus, in view of (1.5), we have

$$
\mathcal{E}_{\infty}^{\prime}(G)=\mathcal{E}^{\prime}(G) \cap \mathscr{D}_{\infty}^{\prime}(G) .
$$

That is, $\mathcal{E}_{\infty}^{\prime}(G)$ consists of all distributions in $\mathscr{D}_{\infty}^{\prime}(G)$ with compact support.

Since $\mathscr{D}(G)$ and $\mathscr{D}_{\infty}^{(*)}(G)$ are bornological, $\mathscr{D}^{\prime}(G)$ and $\mathscr{D}_{\infty}^{\prime}(G)$ are complete. The completeness of $\mathcal{E}^{\prime}(G)$ was proved in [5].

Proposition 1.5. The space $\mathcal{E}_{\infty}^{\prime}(G)$ is complete.

Proof. We use Grothendieck's completion theorem. Let $T$ be a linear form of $\mathcal{E}_{\infty}(G)$ which is $\sigma\left(\mathcal{E}_{\infty}(G), \mathcal{E}_{\infty}^{\prime}(G)\right)$-continuous on each $\boldsymbol{\tau}_{p}$-bounded subset. Then, in view of (1.5), the restriction of $T$ to $\mathcal{E}(G)$ (resp. $\mathscr{D}_{\infty}^{(*)}(G)$ ) is $\sigma\left(\mathcal{E}(G), \mathcal{E}^{\prime}(G)\right.$ )continuous (resp. $\sigma\left(\mathscr{D}_{\infty}(G), \mathscr{D}_{\infty}^{\prime}(G)\right)$-continuous) on each of its bounded subsets. Since $\mathcal{E}^{\prime}(G)$ and $\mathscr{D}_{\infty}^{\prime}(G)$ are complete, this implies that $T \in \mathcal{E}^{\prime}(G) \cap \mathscr{D}_{\infty}^{\prime}(G)\left(=\mathcal{E}_{\infty}^{\prime}(G)\right)$. Hence the assertion. q.e.d.

Each complex Radon measure $\mu$ on $G$ belongs to $\mathscr{D}_{\infty}^{\prime}(G)$ as distribution since $\varphi \in \mathscr{D}_{\infty}^{(*)}(G) \mapsto \int_{G} \varphi(x) d \mu(x) \in C$ is continuous. If $\mu$ is compactly supported, then $\mu \in \mathcal{E}_{\infty}^{\prime}(G)$. Each $f \in L_{\text {loc }}^{1}\left(G, d_{G}\right)$ is identified with the measure $f d_{G}, d_{G}$ denoting a fixed left Haar measure.

Lemma 1.23. $\mathcal{E}_{\infty}^{\prime}(G)$ is dense in each of $\mathscr{D}^{\prime}(G), \mathscr{D}_{\infty}^{\prime}(G)$ and $\mathcal{E}^{\prime}(G)$.

Proof. Since $\mathscr{D}(G)$ and $\mathcal{E}(G)$ are Montel (hence refloxive), it is easy to check that $\mathscr{D}(G)\left(\subseteq \mathcal{E}_{\infty}^{\prime}(G)\right)$ is dense in each of $\mathscr{D}^{\prime}(G)$ and $\mathcal{E}^{\prime}(G)$ (see [5], p. 53). Hence a fortiori so is $\mathcal{E}_{\infty}^{\prime}(G)$. Now let $T \in \mathscr{D}_{\infty}^{\prime}(G)$. For each $C \in \operatorname{Com}(G)$, choose $a_{C} \in \mathscr{D}(G)$ s.t. $a_{C}(x) \equiv 1$ on $C$. Then the map $T_{C}: f \in \mathcal{E}_{\infty}^{(p)}(G) \mapsto\left\langle a_{C} f, T\right\rangle \in C$ is continuous, i. e., $T_{C} \in \mathcal{E}_{\infty}^{\prime}(G)$. While, since each bounded subset of $\mathscr{D}_{\infty}^{(*)}(G)$ lies in $\mathscr{D}_{\infty}(G ; C)$ for some $C \in \operatorname{Com}(G)$, the net $\left\{T_{C} ; C \in \operatorname{Com}(G)\right\}$ converges to $T$ 
in $\mathscr{D}_{\infty}^{\prime}(G)$. Thus $\mathcal{E}_{\infty}^{\prime}(G)$ is dense in $\mathscr{D}^{\prime}(G)$. $\quad$ q. e. d.

Proposition 1.6. Suppose $G$ is not locally connected. Then, (i) $\mathscr{D}_{\infty}^{\prime}(G) \subsetneq \mathscr{D}^{\prime}(G)$. (ii) The topology of $\mathscr{D}_{\infty}^{\prime}(G)$ is strictly finer than that of $\mathscr{D}^{\prime}(G)$ relativized there. (iii) $\mathcal{E}_{\infty}^{\prime}(G) \subsetneq \mathcal{E}^{\prime}(G)$. (iv) The topology of $\mathcal{E}_{\infty}^{\prime}(G)$ is strictly finer than that of $\mathcal{E}^{\prime}(G)$ relativized there.

Proof. Obviously (i) is implied by (iii). Since $\mathscr{D}_{\infty}^{\prime}(G)$ (resp. $\mathcal{E}_{\infty}^{\prime}(G)$ ) is complete and dense in $\mathscr{D}^{\prime}(G)$ (resp. $\mathcal{E}^{\prime}(G)$ ), (ii) (resp. (iv)) is implied by (i) (resp. (iii)). Therefore our task is to prove (iii). Here, in virtue of Remarks 1.4 and 1.7 (recall that the dual of a direct product is the direct sum of the dual), it suffices to cosider the case $G$ is Lie-projective.

(I) First let $G$ be compact and totally disconnected. Then $\mathscr{D}(G)(=\mathcal{E}(G))$ consists of all trigonometric polynomials on $G\left([6]\right.$, Lemma 3.11) and $\mathscr{D}_{\infty}^{(*)}(G)$ $\left(=\mathcal{E}_{\infty}^{(p)}(G)\right)$ coincides with the space $\mathcal{E}_{0}(G)$. The group $G$ is not finite since it is not locally connected. Hence neither is its unitary dual $\hat{G}$. So we can choose an infinite sequence $\sigma_{k}(k=1,2,3, \cdots)$ of mutually distinct elements of $\hat{G}$. For each $k$ let $d_{k}$ (resp. $\chi_{k}$ ) denotes the degree (resp. character) of $\sigma_{k}$. Choose a sequence $c_{k}$ of positive numbers s.t.

$$
\sum_{k=1}^{\infty} c_{k} d_{k}<\infty
$$

Let $N \in \boldsymbol{H}_{0}(G)$. Since $(G / N)^{\wedge}=A(\hat{G}, N)$, the annihilator of $N$ in $\hat{G}$, the Fourier series of each $\varphi \in \mathscr{D}_{\infty}(G, N)\left(\cong \mathscr{D}_{\infty}(G / N)\right)$ is constructed by the coordinate functions of the elements of $A(\hat{G}, N)$. So $\int_{G} \varphi \bar{\chi}_{k} d_{G}=0$ unless $\sigma_{k} \in A(\hat{G}, N)$. Here, from assumption on $G$, the group $G / N$ is finite, hence so is $A(\hat{G}, N)$. Therefore we can defite a $T \in \mathscr{D}^{\prime}(G)\left(=\mathcal{E}^{\prime}(G)\right)$ by

$$
\langle\varphi, T\rangle=\sum_{k=1}^{\infty} c_{k}^{-1} \int_{G} \varphi(x) \bar{\chi}_{k}(x) d_{G} x \quad(\varphi \in \mathscr{D}(G)) .
$$

Now put $\varphi_{n}=\sum_{k=1}^{n} c_{k} \chi_{k}(\in \mathscr{D}(G))$ for $n=1,2,3, \cdots$, and $\varphi_{0}=\sum_{k=1}^{\infty} c_{k} \chi_{k}\left(\in \mathcal{E}_{0}(G)\right)$ (recall (1.7)). Then $\varphi_{n} \rightarrow \varphi_{0}(n \rightarrow \infty)$ in the space $\mathcal{E}_{0}(G)$. But $\left\langle\varphi_{n}, T\right\rangle=n \rightarrow \infty$. This shows that $T \in \mathcal{E}^{\prime}(G) \backslash \mathcal{E}_{\infty}^{\prime}(G)$.

(II) Next let $G$ be finite-dimensional. Then $G$ is locally isomorphic to $(G / N) \times N$ for a totally disconnected $N \in \boldsymbol{H}_{0}(G)$. Since $G$ is not locally connected, neither is $N$. Therefore, in view of (I), we have a $T_{0} \in \mathscr{D}^{\prime}(N) \backslash \mathscr{D}_{\infty}^{\prime}(N)$, a sequence $\phi_{n}(n=1,2,3, \cdots)$ in $\mathscr{D}(N)$ and $\phi_{0} \in \mathcal{E}_{0}(N)$ such that $\phi_{n} \rightarrow \phi_{0}$ in $\mathcal{E}_{0}(N)$ but $\left\langle\psi_{n}, T_{0}\right\rangle \rightarrow \infty$. The restricton map $\left.\varphi \in \mathscr{D}(G) \mapsto \varphi\right|_{N}$ carries $\mathscr{D}(G)$ into $\mathscr{D}(N)$ continuously because it carries each $\mathscr{D}_{\infty}^{(+)}\left(G, N_{1} ; C\right)\left(\left(N_{1}, C\right) \in \boldsymbol{H}_{0}(G) \times \operatorname{Com}(G)\right)$ into $\mathscr{D}_{\infty}^{(+)}\left(N, N_{1} \cap N\right), N_{1} \cap N$ belonging to $\boldsymbol{H}_{0}(N)$, continuously. Hence we can define a $T \in \mathscr{D}^{\prime}(G)$ by $\langle\varphi, T\rangle=\left\langle\left.\varphi\right|_{N}, T_{0}\right\rangle(\varphi \in \mathscr{D}(G))$. 'Then $T \in \mathcal{E}^{\prime}(G)$ since $\operatorname{supp}(T) \leqq N$. Let $\dot{\phi}_{n}, \dot{\phi}_{0}$ be the images of $\phi_{n}, \phi_{0}$ (resp.) in $\mathscr{D}_{\infty}(G)$ by the map of Lemma 1.22 . Then $\dot{\phi}_{n} \in \mathscr{D}(G)$ and $\dot{\phi}_{n} \rightarrow \dot{\phi}_{0}$ in $\mathscr{D}_{\infty}^{(*)}(G)$. But $\left\langle\dot{\psi}_{n}, T\right\rangle=\left\langle\dot{\psi}_{n}, T_{0}\right\rangle \rightarrow \infty$. Thus, $T \notin$ $\mathscr{D}_{\infty}^{\prime}(G)$, hence $T \in \mathcal{E}^{\prime}(G) \backslash \mathcal{E}_{\infty}^{\prime}(G)$. 
(III) We consider the general case. Since $G$ is not locally connected, there exists an $N \in \boldsymbol{H}(G) \backslash \boldsymbol{H}_{0}(G)$. Then, by (II), one has a compactly supported $T_{1} \in$ $\mathscr{D}^{\prime}(G / N) \backslash \mathscr{D}_{\infty}^{\prime}(G / N)$. Analogously to the proof of Lemma 1.11 we can show that the map $g \in \mathscr{D}(G / N) \mapsto g \circ \pi_{N}^{G}$ is a topological linear isomorphism of $\mathscr{D}(G / N)$ onto $\mathscr{D}(G, N)=\left\{\varphi \in \mathscr{D}(G) ;{ }_{y} \varphi=\varphi(y \in N)\right\}$, viewed as topological linear subspace of $\mathscr{D}(G)$. Thus we can regard $T_{1}$ as a continuous, but not $\tau_{*}$-continuous, linear form of $\mathscr{D}(G, N)$. $\rho_{N}$ maps each $\mathscr{D}_{\infty}^{(*)}\left(G, N_{1}\right)\left(N_{1} \in \boldsymbol{H}_{0}(G)\right)$ into itself continuously (Lemma 1.10) and so induces a continuous projection of $\mathscr{D}(G)$ onto $\mathscr{D}(G, N)$ (see Remark 1.5, (ii)). So we can now define a $T \in \mathscr{D}^{\prime}(G)$ by $\langle\varphi, T\rangle=\left\langle\rho_{N}(\varphi), T_{1}\right\rangle$ $(\varphi \in \mathscr{D}(G))$. Then $T \in \mathcal{E}^{\prime}(G)$ since $\operatorname{supp}(T) \cong\left(\pi_{N}^{G}\right)^{-1}\left(\operatorname{supp}\left(T_{1}\right)\right)$. But $T \notin \mathscr{D}_{\infty}^{\prime}(G)$ since it is not $\tau_{*}$-continuous on $\mathscr{D}(G, N)$ (one has $T=T_{1}$ on $\mathscr{D}(G, N)$ ). The proof is now complete. q.e.d.

1.6. Left invariant continuous linear maps on the spaces $\mathcal{E}(G)$ and $\mathcal{E}_{\infty}^{(p)}(G)$. We conclude this chapter by showing here that the elements of $\mathcal{E}^{\prime}(G)$ (resp. $\left.\mathcal{E}_{\infty}^{\prime}(G)\right)$ are in correspondence with the left invariant continuous linear maps on $\mathcal{E}(G)$ (resp. $\mathcal{E}_{\infty}^{(p)}(G)$ ).

Lemma 1.24. (i) Let $T \in \mathscr{D}^{\prime}(G)\left(\right.$ resp. $\left.\mathscr{D}_{\infty}^{\prime}(G)\right)$. The linear map $\varphi \rightarrow\left\langle\varphi(x \cdot), T_{x}\right\rangle$ carries $\mathscr{D}(G)$ (resp. $\mathscr{D}_{\infty}^{(*)}(G)$ ) into $\mathcal{E}(G)$ (resp. $\mathcal{E}_{\infty}^{(p)}(G)$ ) continuously.

(ii) If $T \in \mathcal{E}^{\prime}(G)$ (resp. $\mathcal{E}_{\infty}^{\prime}(G)$ ), the above map carries each of $\mathscr{D}(G)$ and $\mathcal{E}(G)$ (resp. $\mathscr{D}_{\infty}^{(*)}(G)$ and $\mathcal{E}_{\infty}^{(p)}(G)$ ) into itself continuously, too.

(i) and (ii) are valid even if $\left\langle\varphi(x \cdot), T_{x}\right\rangle$ is replaced by $\left\langle\varphi(\cdot y), T_{y}\right\rangle$.

Proof. We only prove here the assertions (i) for $T \in \mathscr{D}_{\infty}^{\prime}(G)$ and (ii) for $T \in \mathcal{E}_{\infty}^{\prime}(G)$. The other assertions are verified analogously.

(i) for $T \in \mathscr{D}_{\infty}^{\prime}(G)$ : Let $\varphi \in \mathscr{D}_{\infty}(G)$. In virtue of Lemma 1.15, the function $y \in G \mapsto\left\langle\varphi\langle x y), T_{x}\right\rangle$ is continuous. Besides, for each $\alpha \in R(G)$ and $y \in G$, we have by the mean value theorem

$$
\left.\frac{1}{t}\left\langle\varphi(x y \alpha(t)), T_{x}\right\rangle-\left\langle\varphi(x y), T_{x}\right\rangle\right\}=\left\langle d_{\alpha}^{(r)} \varphi(x y \alpha(s)), T_{x}\right\rangle,
$$

where $|s|\left\langle|t|\right.$. Hence, plainly, $d_{\alpha}^{(r)}\left\langle\varphi(x \cdot), T_{x}\right\rangle$ exists and equals $\left\langle d_{\alpha}^{(r)} \varphi(x \cdot), T_{x}\right\rangle$. This observation immediately shows that $\left\langle\varphi(x \cdot), T_{x}\right\rangle$ belongs to $\mathcal{E}_{\infty}(G)$ and

$$
d_{\alpha_{1}}^{(r)} \cdots d_{\alpha_{p}}^{(r)}\left\langle\varphi(x \cdot), T_{x}\right\rangle=\left\langle d_{\alpha_{1}}^{(r)} \cdots d_{\alpha_{p}}^{(r)} \varphi(x \cdot), T_{x}\right\rangle
$$

holds for any $\alpha_{1}, \cdots, \alpha_{p} \in R(G)(p=1,2,3, \cdots)$. We now show that the map $\varphi \in \mathscr{D}_{\infty}^{(*)}(G) \mapsto\left\langle\varphi(x \cdot), T_{x}\right\rangle \in \mathcal{E}_{\infty}^{(p)}(G)$ is continuous. Take a pro-Lie open subgroup $G_{1}$ of $G$. Let $(N, C) \in \boldsymbol{H}\left(G_{1}\right) \times \operatorname{Com}(G)$, and $\varphi_{\lambda}$ be a net in $\mathscr{D}_{\infty}^{(r)}(G, N ; C)_{\rho}$ converging to 0 . Our task is to show that, for each $a \in \mathscr{D}_{\infty}(G)$, the net $a\left\langle\varphi_{\lambda}(x \cdot), T_{x}\right\rangle$ converges to 0 in $\mathscr{D}_{\infty}^{(*)}(G)$. In view of Lemma 1.17, the net $d_{\alpha_{1}}^{(r)} \cdots d_{\alpha_{p}}^{(r)} \varphi_{\lambda}$ converges to 0 in $\mathscr{D}_{\infty}^{(r)}(G, N ; C)_{\rho}$ for any $\alpha_{1}, \cdots, \alpha_{p} \in R(G)(p=1,2,3, \cdots)$. Since the right regular representation of $G$ on $\mathscr{D}_{\infty}^{(*)}(G)$ is continuous (1.3), it then follows that $\left(d_{\alpha_{1}}^{(r)} \cdots d_{\alpha_{p}}^{(r)} \varphi_{\lambda}\right)_{y} \rightarrow 0$ in $\mathscr{D}_{\infty}^{(*)}(G)$ uniformly w.r.t. $y \in \operatorname{supp}(a)$. Hence $\left\langle d_{\alpha_{1}}^{(r)} \cdots d_{\alpha_{p}}^{(r)} \varphi_{\lambda}(x y), T_{x}\right\rangle \rightarrow 0$ uniformly w.r.t. $y \in \operatorname{supp}(a)$. By the Leibniz formula 
and (1.9) it now follows that $a\left\langle\varphi_{\lambda}(x \cdot), T_{x}\right\rangle$ converges to 0 in $\mathscr{D}_{\infty}^{(r)}\left(G, N^{\prime}\right.$; $\operatorname{supp}(a))_{\rho}$, hence in $\mathscr{D}_{\infty}^{(*)}(G)$, where $N^{\prime}$ is chosen in $\boldsymbol{H}_{0}\left(G_{1}\right)$ so that $N^{\prime} \leqq N$ and $a \in \mathscr{D}_{\infty}\left(G, N^{\prime}\right)$.

(ii) for $T \in \mathcal{E}_{\infty}^{\prime}(G)$ : Choose $b \in \mathscr{D}(G)$ s.t. $b(x) \equiv 1$ on a neighbourhood of $\operatorname{supp}(T)$. By what has been shown, the map $\varphi \mapsto\left\langle\varphi(x \cdot), T_{x}\right\rangle\left(=\left\langle b(x) \varphi(x \cdot), T_{x}\right\rangle\right)$ carries each $\mathscr{D}_{\infty}^{(r)}(G, N ; C)_{\rho}\left((N, C) \in \boldsymbol{H}\left(G_{1}\right) \times \operatorname{Com}(G)\right)$ into $\mathscr{D}_{\infty}^{(p)}\left(G ;(\operatorname{supp}(b))^{-1} C\right)$ continuously. Since $\tau_{p}=\tau_{*}$ on $\mathscr{D}_{\infty}\left(G ;(\operatorname{supp}(b))^{-1} C\right)$, this shows that $\mathscr{D}_{\infty}^{(*)}(G)$ is carried into itself continuously under the above map. Next we show that $\mathcal{E}_{\infty}^{(p)}(G)$ is also carried into itself contiuously. Let $a \in \mathscr{D}_{\infty}(G)$. Choose $c \in \mathscr{D}(G)$ s.t. $c(x) \equiv 1$ on $\operatorname{supp}(b) \cdot \operatorname{supp}(a)$. Then, for $\varphi \in \mathcal{E}_{\infty}(G), a\left\langle\varphi(x \cdot), T_{x}\right\rangle=\langle a(\cdot) b(x) \varphi(x \cdot)$, $\left.T_{x}\right\rangle=a\left\langle c(x \cdot) \varphi(x \cdot), T_{x}\right\rangle$. Hence, in view of what has been shown, one sees that the map $\varphi \rightsquigarrow a\left\langle\varphi(x \cdot), T_{x}\right\rangle$ is ontinuous from $\mathcal{E}_{\infty}^{(p)}(G)$ into $\mathscr{D}_{\infty}^{(*)}(G)$. Since $a$ is arbitrary, the proof is complete. q.e.d.

Let $S, T \in \mathscr{D}^{\prime}(G)$, and suppose one of them has compact support. Then the convolution $S * T\left(\in \mathscr{D}^{\prime}(G)\right)$ is defined, for which

$$
\begin{aligned}
\langle\varphi, S * T\rangle & =\left\langle\left\langle\varphi(x \cdot), S_{x}\right\rangle, T\right\rangle \\
& =\left\langle\left\langle\varphi(\cdot y), T_{y}\right\rangle, S\right\rangle \quad(\varphi \in \mathscr{D}(G))
\end{aligned}
$$

holds ([5]). It is not difficult to check

$$
\operatorname{supp}(S * T) \subseteq \operatorname{supp}(S) \cdot \operatorname{supp}(T) .
$$

Using (1.10) and Lemma 1.24, we can see that if the above $S, T$ belong to $\mathscr{D}_{\infty}^{\prime}(G)$, then $S * T \in \mathscr{D}_{\infty}^{\prime}(G)$. In this case $(1.10)$ holds for $\varphi \in \mathscr{D}_{\infty}(G)$. Similarly, if $S, T \in \mathcal{E}^{\prime}(G)$ (resp. $\mathcal{E}_{\infty}^{\prime}(G)$ ), then $S * T \in \mathcal{E}^{\prime}(G)$ (resp. $\mathcal{E}_{\infty}^{\prime}(G)$ ) and (1.10) holds for $\varphi \in \mathcal{E}(G)$ (resp. $\mathcal{E}_{\infty}(G)$ ). By the last fact it can be seen that each of $\mathcal{E}^{\prime}(G)$ and $\mathcal{E}_{\infty}^{\prime}(G)$ is a topological algebra under the convolution product and contains $\delta_{e}$, the Dirac measure at $e$, as its identity.

The modular function $\Delta_{G}$ belongs to $\mathcal{E}(G)$. Indeed, choose $\varphi \in \mathscr{D}(G)$ s.t. $\int_{G} \check{\varphi} d_{G}=1$. Then

$$
\Delta_{G}(x)=\int_{G} \Delta_{G}(x) \check{\varphi}(y) d_{G} y=\int_{G} \varphi\left(x y^{-1}\right) d_{G} y \quad(x \in G) .
$$

Since $\varphi \in \mathscr{D}_{\infty}(G, N)_{\sigma}$ for some $N \in \boldsymbol{H}_{0}\left(G_{1}\right), G_{1}$ being a pro-Lie open subgroup of $G$, this shows that $\Delta_{G} \in \mathcal{E}_{\infty}(G, N)_{\sigma}(\subseteq \mathcal{E}(G))$. Now let us define an involutive map $T \mapsto \breve{T}$ on $\mathscr{D}^{\prime}(G)$ as the adjoint of the topological linear automorphism $\varphi \mapsto$ $\left(\Delta_{G} \varphi\right)^{\prec}$ of $\mathscr{D}(G):\langle\varphi, \check{T}\rangle=\left\langle\left(\Delta_{G} \varphi\right)^{2}, T\right\rangle\left(\varphi \in \mathscr{D}(G), T \in \mathscr{D}^{\prime}(G)\right)$. (Notice that $\left(f d_{G}\right)^{2}$ $=\check{f} d_{G}$ holds for $f \in L_{\mathrm{loc}}^{1}\left(G, d_{G}\right)$.) Then, obviously,

$$
\operatorname{supp}(\check{T})=(\operatorname{supp}(T))^{-1} \quad\left(T \in \mathscr{D}^{\prime}(G)\right) .
$$

Since the map $\varphi \mapsto \check{\varphi}$ is continuous also on $\mathscr{D}_{\infty}^{(*)}(G)$, we see that if $T \in \mathscr{D}_{\infty}^{\prime}(G)$, then $\check{T} \in \mathscr{D}_{\infty}^{\prime}(G)$. For the same reason, if $T \in \mathcal{E}^{\prime}(G)\left(\right.$ resp. $\mathcal{E}_{\infty}^{\prime}(G)$ ), then $\check{T} \in \mathcal{E}^{\prime}(G)$ (resp. $\mathcal{E}_{\infty}^{\prime}(G)$ ). 
Lemma 1.25. Let $\mathcal{K}$ designate $\mathscr{D}(G), \mathscr{D}_{\infty}^{(*)}(G), \mathcal{E}(G)$ or $\mathcal{E}_{\infty}^{(p)}(G)$. Then, for each $\varphi \in \mathcal{K}$ and $T \in \mathcal{K}^{\prime}$, there holds

$$
\varphi * T=\left\langle\varphi(\cdot y), \check{T}_{y}\right\rangle \quad \text { (as distribution). }
$$

Proof. By the above mentioned remarks, we have, for $\phi \in \mathscr{D}(G)$,

$$
\begin{aligned}
\langle\psi, \varphi * T\rangle & =\left\langle\int_{G} \psi(x y) \varphi(x) d_{G} x, T_{y}\right\rangle \\
& =\left\langle\int_{G} \psi(x) \varphi(x y) d_{G} x, \check{T}_{y}\right\rangle=\langle\varphi, \phi * \check{T}\rangle \\
& =\int_{G} \psi(x)\left\langle\varphi(x y), \check{T}_{y}\right\rangle d_{G} x .
\end{aligned}
$$

Hence $\varphi * T=\left\langle\varphi(\cdot y), \check{T}_{y}\right\rangle$. q.e.d.

Corollary. Let $\mathcal{K}$ be as in Lemma 1.25. For each $\varphi \in \mathcal{K}$ and $T \in \mathcal{K}^{\prime}$, there holds

$$
\langle\varphi, T\rangle=\varphi * \check{T}(e) .
$$

Let $S, T \in \mathscr{D}^{\prime}(G)$, and suppose one of them has compact support. Then, by (1.10) and (1.13),

$$
\langle\varphi, S * T\rangle=\langle\varphi * \check{T}, S\rangle \quad(\varphi \in \mathscr{D}(G)) .
$$

Furthermore, as is easily checked,

$$
(S * T)^{\llcorner}=\check{T} * \check{S} .
$$

New let $\mathcal{K}$ stand for $\mathcal{E}(G)$ or $\mathcal{E}_{\infty}^{(p)}(G)$. In view of Lemmas 1.24 and 1.25 we can define, for each $T \in \mathcal{K}^{\prime}$, a continuous linear map $D_{T}$ on $\mathcal{K}$ as

$$
D_{T} f=f * \check{T} \quad(f \in \mathcal{K}) .
$$

Since $\delta_{x^{-1}} * f={ }_{x} f\left(x \in G, f \in L_{\mathrm{loc}}^{1}\left(G, d_{G}\right)\right.$ ) (use 1.10)), $\delta_{x}$ denoting the Dirac measure at $x$, we see by the associativity law for distributions that $D_{T}$ is left invariant (i. e., $D_{T}\left({ }_{x} f\right)={ }_{x}\left(D_{T} f\right)(x \in G, f \in \mathcal{K})$.

Theorem 1.4. Let $\mathcal{K}$ designate $\mathcal{E}(G)$ or $\mathcal{E}_{\infty}^{(p)}(G)$. The map $T \mapsto D_{T}$ sets $u p$ an isomorphism of the algebra $\mathcal{K}^{\prime}$ onto the algebra of all left invariant continuous linear maps on $\mathcal{K}$. Its inverse map is given by $D \mapsto T_{D}$, where $T_{D}\left(\in \mathcal{K}^{\prime}\right)$ is defined as

$$
\left\langle f, T_{D}\right\rangle=D f(e) \quad(f \in \mathcal{K}) .
$$

$D_{T}$ decreases supports (i.e., $\operatorname{supp}\left(D_{T} f\right) \leqq \operatorname{supp}(f)$ for all $\left.f \in \mathcal{K}\right)$ if and only if $\operatorname{supp}(T) \leqq\{e\}$.

Proof. Denote the map $T \in \mathcal{K}^{\prime} \mapsto D_{T}$ by $\Psi$. It is clear from (1.16) and (1.17) 
that $\Psi$ is an algebra homomorphism. Its injectivity follows from (1.14). Let us see its surjectivity. Take any left invariant continuous linear map $D$ on $\mathcal{K}$. Define the linear map $T_{D}$ on $\mathcal{K}$ by (1.18). Then, plainly, $T_{D} \in \mathcal{K}^{\prime}$. Besides, for any $f \in \mathcal{K}$ and $x \in G$,

$$
\begin{aligned}
D f(x) & =D\left({ }_{x} f\right)(e)=\left\langle{ }_{x} f, T_{D}\right\rangle \\
& ={ }_{x} f * \check{T}_{D}(e) \quad(\text { by }(1.14)) \\
& =D_{T_{D}}\left({ }_{x} f\right)(e)=D_{T_{D}} f(x) .
\end{aligned}
$$

Hence $D=D_{T_{D}}$. This shows that $\Psi$ is surjective and, at the same time, that its inverse map is given by $D \mapsto T_{D}$. Finally, from (1.11), (1.12), (1.17) and (1.18), we see that $D_{T}$ decreases supports if and only if $\operatorname{supp}(T) \leqq\{e\}$. q.e.d.

Theorem 1.4 together with Lemmas 1.24 and 1.25 yields the following

Corollary. Every left invariant continuous linear map on $\mathcal{E}(G)$ (resp. $\mathcal{E}_{\infty}^{(p)}(G)$ ) leaves $\mathscr{D}(G)$ (resp. each of $\mathscr{D}(G), \mathscr{D}_{\infty}^{(*)}(G)$ and $\mathcal{E}(G)$ ) invariant and induces on it a continuous map.

Theorem 1.4 together with Proposition 1.6, (iii) shows that if $G$ is not locally connected, the class of the left invariant continuous linear maps on $\mathcal{E}(G)$ is strictly wider (in the obvious sense) than the class of the maps on $\mathcal{E}_{\infty}^{(p)}(G)$ with the same properties. But we shall see in 2.7 that their subclasses consisting of all support-decreasing elements coincide with each other. As against the present section, we intened to discuss in the next chapter the support-decreasing (not necessarily left invariant) continuous linear maps on the spaces $\varepsilon_{\infty}^{(p)}(G)$, $\mathscr{D}_{\infty}^{(*)}(G), \mathcal{E}(G)$ and $\mathscr{D}(G)$.

\section{Chapter 2. Differential operators}

2.1. Derivations and differential operators. A linear map $d$ on an algebra $\mathcal{E}$ over $\boldsymbol{C}$ is called a derivation if $d(f g)=(d f) g+f(d g)$ holds for $f, g \in \mathcal{E}$.

Lemma 2.1. Let $d$ be a derivation on $\mathcal{E}_{\infty}(G)$ or on $\mathscr{D}_{\infty}(G)$. Then $\operatorname{supp}(d f) \subseteq$ $\operatorname{supp}(f)$ holds for $f \in \mathscr{D}_{\infty}(G)$.

Proof. Take any $f \in \mathscr{D}_{\infty}(G)$ and any neighbourhood $U$ of $\operatorname{supp}(f)$. Choose $a \in \mathscr{D}(G)$ such that $a(x) \equiv 1$ on $\operatorname{supp}(f)$ and $\operatorname{supp}(a) \cong U$ (Lemma 1.18). Then, since $f=a f$, we have $d f=(d a) f+a(d f)$. Hence $\operatorname{supp}(d f) \leqq U$. Since $U$ is arbitrary, the assertion follows. q.e.d.

Lemma 2.1 shows in particular that each derivarion on $\mathcal{E}_{\infty}(G)$ leaves $\mathscr{D}_{\infty}(G)$ invariant and so induces a derivation on it.

Lemma 2.2. If a derivation $d$ on $\mathcal{E}_{\infty}(G)$ is $\boldsymbol{\tau}_{*}$-continuous on $\mathscr{D}_{\infty}(G)$, then it is $\tau_{p}$-continuous on $\mathcal{E}_{\infty}(G)$. 
Proof. Obvious from the equality $\varphi(d f)=d(\varphi f)-(d \varphi) f\left(f \in \mathcal{E}_{\infty}(G), \varphi \in \mathscr{D}_{\infty}(G)\right)$. q.e.d.

Lemma 2.3. Let $D$ be a continuous linear map on $\mathcal{E}_{\infty}^{(p)}(G)$ s.t. $\operatorname{supp}((D f) \subseteq$ $\operatorname{supp}(f)$ for all $f \in \mathscr{D}_{\infty}(G)$. Then,

(i) $D$ induces a continuous linear map on $\mathscr{D}_{\infty}^{(*)}(G)$.

(ii) $\operatorname{supp}(D f) \subseteq \operatorname{supp}(f)$ holds for all $f \in \mathcal{E}_{\infty}(G)$.

Proof. Let $G_{1}$ be a pro-Lie open subgroup of $G$. Take any $(N, C) \in \boldsymbol{H}\left(G_{1}\right)$ $\times \operatorname{Com}(G)$. Then, since $\boldsymbol{\tau}_{*}=\boldsymbol{\tau}_{p}$ on $\mathscr{D}_{\infty}(G ; C)$ (Lemma 1.16), it is clear from assumption that $D$ maps $\mathscr{D}_{\infty}^{(+)}(G, N ; C)$ into $\mathscr{D}_{\infty}^{(*)}(G ; C)$ continuously. Hence the assertion (i) follows. Next, take a locally finite family $\left\{a_{\kappa}\right\}_{\kappa \in K}$ of functions in $\mathscr{D}(G)$ s.t. $\sum_{\kappa \in K} a_{\kappa}(x) \equiv 1$ on $G$ (Lemma 1.19). Let $\boldsymbol{F}=\{F\}$ be the upper directed family of all finite subsets of $K$. Then, for each $f \in \mathcal{E}_{\infty}(G)$, it is plain that the net $\left\{\sum_{\kappa \in F} a_{\kappa} f ; F \in \boldsymbol{F}\right\}$ converges to $f$ in $\mathcal{E}_{\infty}^{(p)}(G)$. Hence it follows that the net $\left\{\sum_{\kappa \in F} D\left(a_{\kappa} f\right) ; F \in \boldsymbol{F}\right\}$ converges to $D f$ relative to $\boldsymbol{\tau}_{p}$, hence $a$ fortiori pointwise. Since $\operatorname{supp}\left(D\left(a_{\kappa} f\right)\right) \cong \operatorname{supp}\left(a_{\kappa} f\right) \cong \operatorname{supp}(f)$ for all $\kappa \in K$, this implies that $\operatorname{supp}(D f) \subseteq \operatorname{supp}(f)$. The assertion (ii) has thus been proved. q.e.d.

Lemma 2.4. Let $D$ be a continuous linear map on $\mathscr{D}_{\infty}^{(*)}(G)$ s.t. $\operatorname{supp}(D f) \cong$ $\operatorname{supp}\left((f)\right.$ for all $f \in \mathscr{D}_{\infty}(G)$. Then $D$ is extended uniquely to a continuous linear map on $\mathcal{E}_{\infty}^{(p)}(G)$. Here, if $D$ is a derivation, this extension is also a derivation on $\mathcal{E}_{\infty}(G)$.

Proof. Let $\varphi, \phi \in \mathscr{D}_{\infty}(G)$. Choose $a \in \mathscr{D}(G)$ so that $a(x) \equiv 1$ on a neighborhood of $\operatorname{supp}(\psi)$. Then, since $\operatorname{supp}(D((1-a) \varphi)) \cong \operatorname{supp}((1-a) \varphi) \leqq \operatorname{supp}(1-a)$ and so $\phi D((1-a) \varphi)=0$, we have $\psi D \varphi=\psi D(a \varphi)$. Hence it follows that the map $\varphi \in \mathscr{D}_{\infty}^{(p)}(G) \mapsto \phi D \varphi \in \mathscr{D}_{\infty}^{(*)}(G)$ is continuous. Since $\psi$ is arbitrary, this shows that $D$ is continuous on $\mathscr{D}_{\infty}(G)$ in regard to the relativized $\tau_{p}$. Since $\mathcal{E}_{\infty}^{(p)}(G)$ is complete and $\mathscr{D}_{\infty}(G)$ is dense in it, the first half of the lemma now follows. The second half is easy to check since $\mathcal{E}_{\infty}^{(p)}(G)$ is a topological algebra. q.e.d.

Let $\boldsymbol{\delta}_{1}$ (resp. $\boldsymbol{\delta}_{2}$ ) denote the totality of continuous derivations on $\mathcal{E}_{\infty}^{(p)}(G)$ (resp. $\mathscr{D}_{\infty}^{(*)}(G)$ ). Then, by Lemmas $2.1,2.3$ and 2.4 , we see that $\boldsymbol{\delta}_{1}$ corresponds bijectively to $\boldsymbol{\delta}_{2}$ through the restriction map $\left.d \in \boldsymbol{\delta}_{1} \rightarrow d\right|_{\mathscr{D}_{\infty}(G)}$. So, in the sequel, we do not distinguish between $\boldsymbol{\delta}_{1}$ and $\boldsymbol{\delta}_{2}$. That is, $d \in \boldsymbol{\delta}_{1}$ and $\left.d\right|_{\mathscr{D}_{\infty}(G)}$ are always identified.

Definition 2.1. Each continuous derivation on $\mathcal{E}_{\infty}^{(p)}(G)$ or, what is the same, on $\mathscr{D}_{\infty}^{(*)}(G)$ is called a continuous derivation on $G$. The totality of continuous derivations on $G$ is denoted by $\boldsymbol{\delta}(G)$.

Let $\overline{\boldsymbol{D}}_{\mathbf{1}}$ (resp. $\overline{\boldsymbol{D}}_{2}$ ) denote the totality of support-decreasing continuous linear maps on $\mathcal{E}_{\infty}^{(p)}(G)$ (resp. $\mathscr{D}_{\infty}^{(*)}(G)$ ). Lemmas 2.3. and 2.4 obviously shows that the restriction map $\left.D \in \overline{\boldsymbol{D}}_{1} \mapsto D\right|_{\mathscr{D}_{\infty}(G)}$ sets up a bijection of $\overline{\boldsymbol{D}}_{1}$ onto $\overline{\boldsymbol{D}}_{2}$. So, as in 
the case of continuous derivations, we always identify $\overline{\boldsymbol{D}}_{1}$ and $\overline{\boldsymbol{D}}_{2}$ through this map. Here note that this identification preserves the left and the right invariancy of maps. That is, a map in $\bar{D}_{1}$ is left or right invariant on $\mathcal{E}_{\infty}(G)$ if and only if so it is on $\mathscr{D}_{\infty}(G)$.

We now make the following

Definition 2.2. Each support-decreasing continuous linear map on $\mathcal{E}_{\infty}^{(p)}(G)$ or, what is the same, on $\mathscr{D}_{\infty}^{(*)}(G)$ is called a differential operator on $G$. The totality of differential operators on $G$ is denoted by $\bar{D}(G)$. The totality of its left (resp. right) invariant members is denoted by $\overline{\boldsymbol{D}}_{l}(G)\left(\operatorname{resp} . \overline{\boldsymbol{D}}_{r}(G)\right.$ ).

Note that $\boldsymbol{\delta}(G)$ is included in $\overline{\boldsymbol{D}}(G)$ (Lemma 2.1). Under the obvious algebra operations $\overline{\boldsymbol{D}}(G)$ becomes an algebra over $\boldsymbol{C}$. Each of $\overline{\boldsymbol{D}}_{l}(G)$ and $\overline{\boldsymbol{D}}_{r}(G)$ is its subalgebra. $\boldsymbol{\delta}(G)$ is a Lie algebra under the linear operation in $\overline{\boldsymbol{D}}(G)$ and the commutator product $\left[d, d^{\prime}\right]=d d^{\prime}-d^{\prime} d$. For each $D \in \overline{\boldsymbol{D}}(G)$, we can define $\check{D} \in \overline{\boldsymbol{D}}(G)$ by $\check{D} f=(D \check{f})^{\vee}\left(f \in \mathcal{E}_{\infty}(G)\right)$. Then the map $D \mapsto \check{D}$ is an algebra automorphism of $\bar{D}(G)$, and carries $\overline{\boldsymbol{D}}_{l}(G)$ and $\overline{\boldsymbol{D}}_{r}(G)$ onto each other. Also, this map induces a Lie algebra automorphism of $\boldsymbol{\delta}(G) . \quad \overline{\boldsymbol{D}}(G)$ includes $\mathcal{E}_{\infty}(G)$, where each element of $\mathcal{E}_{\infty}(G)$ is regarded as a multiplication operator. A map $D \in \bar{D}(G)$ does not necessarily leave $\mathscr{D}(G)$ invariant, but is determined by the behavior on it because of its density in $\mathcal{E}_{\infty}^{(p)}(G)$. Now suppose $D \in \overline{\boldsymbol{D}}_{l}(G)$. Then $D$ leaves $D(G)$ invariant because it leaves each $\mathscr{D}_{\infty}(G, N)_{\sigma}\left(N \in \boldsymbol{H}_{0}\left(G_{1}\right), G_{1}\right.$ a pro-Lie open subgroup of $G$ ) invariant (or by Corallary to Theorem 1.4). Furthermore it leaves $\mathscr{D}\left(G_{1}\right)$, identified with $\mathscr{D}\left(G ; G_{1}\right)$, invariant and is determined by the restriction there (recall Remark 1.6).

\subsection{Derivations associated to one-parameter subgroups.}

Definition 2.3. $R(G)^{c}$ denotes the complexification of the Lie algebra $R(G)$. For each $\alpha+i \beta \in R(G)^{c}(\alpha, \beta \in R(G), i=\sqrt{-1}), d_{\alpha+i_{i}}^{(r)}\left(\right.$ resp. $\left.d_{\alpha+i \beta}^{(l)}\right)$ is defined to be the derivation

$$
f \longmapsto d_{\alpha}^{(r)} f+i d_{\beta}^{(r)} f \quad\left(\text { resp. } f \longmapsto d_{\alpha}^{(l)} f+i d_{\beta}^{(l)} f\right)
$$

on $\mathcal{E}_{\infty}(G)$ (see Lemma 1.1, (i)).

$d_{\alpha+i \beta}^{(r)}$ is continuous on $\mathscr{D}_{\infty}^{(*)}(G)$ because it maps each $\mathscr{D}_{\infty}^{(r)}(G, N ; C)_{\sigma}((N, C)$ $\in \boldsymbol{H}\left(G_{1}\right) \times \operatorname{Com}(G), G_{1}$ a pro-Lie open subgroup of $\left.G\right)$ into itself continuously. Hence, by Lemma 2.2, $d_{\alpha+i, \beta}^{(r)} \in \boldsymbol{\delta}(G)$. Besides, from Lemma 1.1, $d_{\alpha+i \beta}^{(r)} \in \overline{\boldsymbol{D}}_{l}(G)$ and $\left(d_{\alpha+i \beta}^{(r)}\right)^{\vee}=d_{a+i \beta}^{(l)}$.

Theorem 2.1. The map $\alpha+i \beta \mapsto d_{a+i \beta}^{(r)}$ is a Lie algebra isomorphism of $R(G)^{c}$ into $\boldsymbol{\partial}(G)$. The image of this map coincides with the totality of left invariant elements of $\boldsymbol{\delta}(G)$ (i.e., with $\boldsymbol{\delta}(G) \cap \overline{\boldsymbol{D}}_{l}(G)$ ).

Proof. From Lemma 1.4 and Remark 1.1 it is clear that the above map is a Lie algebra homomorphism. Let $G_{1}$ be a pro-Lie open subgroup of $G$. Suppose $d_{\alpha+i_{\beta}}^{(r)}=0$. Then, for any $N \in \boldsymbol{H}_{0}\left(G_{1}\right), g \in \mathscr{D}\left(G_{1} / N\right)$ and $x \in G_{1}$, we have 
by (1.3)

$$
d_{\alpha}^{(r)}{ }_{N}^{+i \beta_{N}} g\left(\pi_{N}^{G 1}(x)\right)=d_{\alpha+i \beta}^{(r)}\left(g \circ \pi_{N}^{G_{1}}\right)(x)=0,
$$

where $\alpha_{N}=\bar{\pi}_{N}^{G_{1}}(\alpha)$ and $\beta_{N}=\bar{\pi}_{N}^{G_{1}}(\beta)$. Hence $d_{\alpha_{N}+i \beta_{N}}^{(r)}=0$. Since $G_{1} / N$ is a Lie group, this implies that $\alpha_{N}=\beta_{N}=0$. Since $N$ is arbitrary, we have thus $\alpha=\beta$ $=0$ (i. e., $\alpha+i \beta=0$ ), which proves the injectivity of the above homomorphism. Next take any $d \in \boldsymbol{\delta}(G) \cap \overline{\boldsymbol{D}}_{l}(G)$. To complete the proof, it suffices to show that $d=d_{\alpha}^{(r)}+i d_{\beta}^{(r)}$ holds on $\mathscr{D}\left(G_{1}\right)$ for some $\alpha, \beta \in R(G)$. Since $d$ makes each $\mathscr{D}_{\infty}\left(G_{1}, N\right)$ $\left(N \in \boldsymbol{H}_{0}\left(G_{1}\right)\right)$ invariant, we obtain a left invariant element $d_{N} \in \boldsymbol{\delta}\left(G_{1} / N\right)$ determined by $\left(d_{N} g\right) \circ \pi_{N}^{G_{1}}=d\left(g \circ \pi_{N}^{G_{1}}\right)\left(g \in \mathscr{D}\left(G_{1} / N\right)\right)$. Then, since $G_{1} / N$ is a Lie group, there exists a unique pair $\left(\alpha_{N}, \beta_{N}\right)$ of elements of $R\left(G_{1} / N\right)$ s.t. $d_{N}=d_{\alpha_{N}}^{(r)}+i d_{\beta_{N}}^{(r)}$. Now suppose $N, N^{\prime} \in \boldsymbol{H}_{0}\left(G_{1}\right)$ and $N \subseteq N^{\prime}$. Take any $g^{\prime} \in \mathscr{D}\left(G_{1} / N^{\prime}\right)$ and put $g=g^{\prime}$ 。 $\pi_{N^{\prime} N}^{G_{1}}\left(\in \mathscr{D}\left(G_{1} / N\right)\right)$. Then

$$
\begin{aligned}
& \left(\left(d_{\alpha_{N}}^{(r)}+i d_{\beta_{N^{\prime}}}^{(r)}\right) g^{\prime}\right) \circ \pi_{N^{\prime}}^{G_{1}} \\
= & \left(d_{N^{\prime}} g^{\prime}\right) \circ \pi_{N^{\prime}}^{G_{1}}=d\left(g^{\prime} \circ \pi_{N^{\prime}}^{G_{1}}\right)=d\left(g \circ \pi_{N}^{G_{1}}\right)=\left(d_{N} g\right) \circ \pi_{N}^{G_{1}} \\
= & \left(\left(d_{\alpha_{N}}^{(r)}+i d_{\beta_{N}}^{(r)}\right) g\right) \circ \pi_{N^{\prime}}^{G_{1}} \\
= & \left(\left(d_{\alpha_{N} N^{\prime}}^{(r)}+i d_{\beta_{N^{\prime} N}}^{(r)}\right) g^{\prime}\right) \circ \pi_{N^{\prime}}^{G_{1}},
\end{aligned}
$$

where $\alpha_{N^{\prime} N}=\bar{\pi}_{N^{\prime} N}^{G_{1}}\left(\alpha_{N}\right)$ and $\beta_{N^{\prime} N}=\bar{\pi}_{N^{\prime} N}^{G_{1}}\left(\beta_{N}\right)$. Hence $d_{\alpha_{N^{\prime}}}^{(r)}+i d_{\beta_{N} N^{\prime}}^{(r)}=d_{\alpha_{N^{\prime} N}}^{(r)}+$ $i d_{\beta_{N^{\prime} N}}^{(r)}$, i. e., $\alpha_{N^{\prime}}=\bar{\pi}_{N^{\prime} N}^{G_{1}}\left(\alpha_{N}\right)$ and $\beta_{N^{\prime}}=\bar{\pi}_{N^{\prime} N}^{G_{1}}\left(\beta_{N}\right)$. This shows that the maps $t \in \boldsymbol{R} \mapsto\left(\alpha_{N}(t)\right)_{N \in H_{0}\left(G_{1}\right)}$ and $t \in \boldsymbol{R} \mapsto\left(\beta_{N}(t)\right)_{N \in H_{0}\left(G_{1}\right)}$ belong to $R\left(\iota_{G_{1}}\left(G_{1}\right)\right)$ (see $\left.(0.1)\right)$. Now put $\alpha(t)=\iota_{G_{1}}^{-1}\left(\left(\alpha_{N}(t)\right)_{N \in H_{0}\left(G_{1}\right)}\right)$ and $\beta(t)=\iota_{G_{1}^{1}}^{-1}\left(\left(\beta_{N}(t)\right)_{N \in H_{0}\left(G_{1}\right)}\right)(t \in R)$. Then $\alpha, \beta$ $\in R\left(G_{1}\right)(=R(G))$. And, evidently, we have $d=d_{\alpha}^{(r)}+i d_{\beta}^{(r)}$ on $\mathscr{D}_{\infty}\left(G_{1}, N\right)$ for all $N \in \boldsymbol{H}_{0}\left(G_{1}\right)$, and hence on $\mathscr{D}\left(G_{1}\right)$. This completes the proof. q.e.d.

\subsection{The enveloping algebra of $R(G)^{c}$.}

Definition 2.4. $\boldsymbol{D}_{l}(G)$ (resp. $\boldsymbol{D}_{r}(G)$ ) denotes the subalgebra of $\overline{\boldsymbol{D}}(G)$ generated by all the derivations $d_{\alpha+i \beta}^{(r)}$ (resp. $\left.d_{\alpha+i \beta}^{(l)}\right)(\alpha, \beta \in R(G))$ and 1 , the identity operator on $\mathcal{E}_{\infty}(G)$.

Each $D \in \boldsymbol{D}_{l}(G)$ (resp. $\left.\boldsymbol{D}_{r}(G)\right)$ is expressed as

$$
\begin{gathered}
D=\sum c_{\alpha_{1}, \ldots, \alpha_{p}} d_{\alpha_{1}}^{(r)} \cdots d_{\alpha_{p}}^{(r)} \\
\text { (resp. } \left.D=\sum c_{\alpha_{1}, \ldots, \alpha_{p}} d_{\alpha_{1}}^{(l)} \cdots d_{\alpha_{p}}^{(l)}\right) \quad \text { (finite sum), }
\end{gathered}
$$

where $\alpha_{1}, \cdots, \alpha_{p} \in R(G), c_{\alpha_{1}, \ldots, \alpha_{p}} \in \boldsymbol{C}$ and, for the case $p=0, d_{c_{1}}^{(r)} \cdots d_{\alpha_{p}}^{(r)}$ (resp. $\left.d_{\alpha_{1}}^{(l)} \cdots d_{\alpha_{p}}^{(l)}\right)$ means the identity operator 1 . The automorphism $D \mapsto \check{D}$ of $\bar{D}(G)$ maps $\boldsymbol{D}_{l}(G)$ and $\boldsymbol{D}_{r}(G)$ onto each other. $\boldsymbol{D}_{l}(G)$ is of course contained in $\overline{\boldsymbol{D}}_{l}(G)$ but, as will be seen in $\mathbf{2 . 9}$, does not in general coincide with it.

Definition 2.5. $\boldsymbol{U}(G)$ denotes the universal enveloping algebra of the Lie algebra $R(G)^{c}$. $\quad C$ and $R(G)^{c}$ are identified with their canonical images in $\boldsymbol{U}(G)$.

Theorem 2.2. The map $\alpha+i \beta \mapsto d_{u+i \beta}^{(r)}$ of $R(G)^{c}$ into $\boldsymbol{D}_{l}(G)$ extends uniquely 
to an algebra isomorphism of $\boldsymbol{U}(G)$ onto $\boldsymbol{D}_{l}(G)$ mapping the identity of $\boldsymbol{U}(G)$ to that of $\boldsymbol{D}_{l}(G)$.

This theorem is well known if $G$ is a Lie group, and makes the algebra structure of $\boldsymbol{D}_{l}(G)$ clear. By Theorem 2.1, the map $\alpha+i \beta \mapsto d_{\alpha+i \beta}^{(r)}$ is a Lie algebra homomorphism of $R(G)^{c}$ into $\boldsymbol{D}_{l}(G)$, viewed as Lie algebra under the commutator product. Therefore it extends uniquely to an algebra homomorphism of $\boldsymbol{U}(G)$ into $\boldsymbol{D}_{l}(G)$ mapping 1 to 1 . Denote this extension by $\Phi_{G}$. It is then evident that $\Phi_{G}$ is surjective. So, for the proof of the theorem, it remains only to show that $\Phi_{G}$ is injective.

Notation. For any finite number of linearly independent elements $\alpha_{1}, \cdots$, $\alpha_{n}$ of $R(G)$ and positive integers $\mu_{1}, \cdots, \mu_{n}$, we denote by $u\left(\Pi_{i=1}^{n} \alpha_{i}^{\mu_{i}}\right)$ the element of $\boldsymbol{U}(G)$ which corresponds canonically to the element $\Pi_{i=1}^{n} \alpha_{i}^{\mu_{i}}$ of the symmetric algebra of $R(G)^{c}$. (That is, $u\left(\Pi_{i=1}^{n} \alpha_{i}^{\mu_{i}}\right.$ ) is formally the coefficient of $\prod_{i=1}^{n} A_{i}^{\mu_{i}}$ multiplied by $\Pi\left(\mu_{i} !\right) /\left(\sum \mu_{i}\right) !$ in the expansion of $\left(\sum_{i=1}^{n} \alpha_{i} A_{i}\right)^{\Sigma \mu_{i}}, A_{i}$ being supposed to commute with each other and with every $\alpha_{i}$.) Here we include the case $n=0$ (i. e., $\left.\left\{\alpha_{1}, \cdots, \alpha_{n}\right\}=\varnothing\right)$ and define for this case $u\left(\Pi_{i=1}^{n} \alpha_{i}^{\mu_{i}}\right)$ to be the identity element 1 . We denote $\Phi_{G}\left(u\left(\prod_{i=1}^{n} \alpha_{i}^{\mu_{i}}\right)\right)$ by $D\left(\Pi_{i=1}^{n} \alpha_{i}^{\mu_{i}}\right)$.

Now let us take an algebraic linear base $\left\{\gamma_{i}\right\}_{i \in I^{\prime}}$ of $R(G)$. (We do use here the symbol " $I$ '". " $I$ " is put aside for another use in the next section.) We denote by $\tilde{I}^{\prime}$ the totality of the elements $\nu=\left(\nu_{i}\right)_{i \in I^{\prime}}$ of $\boldsymbol{Z}_{+}^{I^{\prime}}, \boldsymbol{Z}_{+}$denoting the set of all non-negative integers, such that $\nu_{i}=0$ for all but at most a finite number of $i \in I^{\prime}$. For each $\nu \in \tilde{I}^{\prime}$, put $u_{\nu}=u\left(\Pi_{\nu_{i} \neq 0} \gamma_{i}^{\nu} i\right)$ and $D_{\nu}=D\left(\Pi_{\nu_{i} \neq 0} \gamma_{i}^{\nu} i\right)(=$ $\Phi_{G}\left(u_{\nu}\right)$ ). Then $\left\{u_{\nu} ; \nu \in \tilde{I}^{\prime}\right\}$ is an algebraic linear base of $\boldsymbol{U}(G)$. Therefore, to complete the proof of Theorem 2.2, it suffices to show that $D_{\nu}\left(\nu \in \tilde{I}^{\prime}\right)$ are linearly independent in $\boldsymbol{D}_{l}(G)$.

Lemma 2.5 Suppose $G$ is Lie-projective. For any finite number of linearly independent elements $\alpha^{(1)}, \cdots, \alpha^{(n)}$ of $R(G)$, there exists an $N \in \boldsymbol{H}_{0}(G)$ such that $\alpha_{N}^{(i)}=\bar{\pi}_{N}^{G}\left(\alpha^{(i)}\right)(i=1, \cdots, n)$ are linearly independent in $R(G / N)$.

Proof. We prove this by finite induction. Assume that, for some $k<n$, there has been chosen an $N_{k} \in \boldsymbol{H}_{0}(G)$ such that $\alpha_{N_{k}}^{(i)}(i=1, \cdots, k)$ are linearly independent in $R\left(G / N_{k}\right)$. This assumption obviously holds for $k=1$. Now, if $\alpha_{N_{k}}^{(i)}(i=1, \cdots, k, k+1)$ are linearly independent in $R\left(G / N_{k}\right)$, we choose $N_{k}$ as $N_{k+1}$. Otherwise, $\alpha_{N_{k}}^{(k+1)}$ is expressed uniquely as

$$
\alpha_{N_{k}}^{(k+1)}=\sum_{i=1}^{k} c_{i} \alpha_{N_{k}}^{(i)} \quad\left(c_{i} \in \boldsymbol{R}\right)
$$

But, since $\alpha^{(i)}(i=1, \cdots, k, k+1)$ are linearly independent in $R(G)$, there exists an $N \in \boldsymbol{H}_{0}(G)$ such that

$$
\alpha_{N}^{(k+1)} \neq \sum_{i=1}^{k} c_{i} \alpha_{N}^{(i)} .
$$


Put $N_{k+1}=N_{k} \cap N$. Then $\alpha_{N_{k+1}}^{(i)}(i=1, \cdots, k, k+1)$ must be linearly independent in $R\left(G / N_{k+1}\right)$ because we have $\alpha_{N_{k}}^{(i)}=\bar{\pi}_{N_{k} N_{k+1}}^{G}\left(\alpha_{N_{k+1}}^{(i)}\right)$ and $\alpha_{N}^{(i)}=\bar{\pi}_{N N_{k+1}}^{G}\left(\alpha_{N_{k+1}}^{(i)}\right)$ for any $i$. The induction is now complete. q.e.d.

Proof of Theorem 2.2. We prove the linear independency of $\left\{D_{\nu} ; \nu \in \tilde{I}^{\prime}\right\}$. Let $\left\{\nu^{(1)}, \cdots, \nu^{(m)}\right\}$ be any finite subset of $\tilde{I}^{\prime}$. Put $J=\left\{i \in I^{\prime} ; \nu_{i}^{(k)} \neq 0\right.$ for some $k(=1, \cdots, m)\}$. Take a pro-Lie open subgroup $G_{1}$ of $G$. Then, by Lemma 2.5, there exists an $N \in \boldsymbol{H}_{0}\left(G_{1}\right)$ such that $\bar{\pi}_{N^{1}}^{G_{1}}\left(\gamma_{i}\right)(i \in J)$ are linearly independent in $R\left(G_{1} / N\right)$. Each $D_{\nu}\left(\nu \in \tilde{I}^{\prime}\right)$ makes $\mathscr{D}_{\infty}\left(G_{1}, N\right)$ invariant and so induces a map $\bar{D}_{\nu}$ on $\mathscr{D}\left(G_{1} / N\right)$ s. t. $\left(\bar{D}_{2} g\right) \circ \pi_{N^{1}}^{G_{1}}=D_{2}\left(g \circ \pi_{N^{1}}\right)\left(g \in \mathscr{D}\left(G_{1} / N\right)\right)$. Evidently, the Lie algebra homomorphism $\bar{\pi}_{N}^{G_{1}}$ of $R\left(G_{1}\right)\left(=R(G)\right.$ ) into $R\left(G_{1} / N\right)$ extends uniquely to an algebra homomorphism of $\boldsymbol{U}\left(G_{1}\right)(=\boldsymbol{U}(G))$ into $\boldsymbol{U}\left(G_{1} / N\right)$ mapping 1 to 1 . Denote this extension again by $\bar{\pi}_{N}^{G_{1}}$. Then, obviously, $\bar{D}_{\nu}=\Phi_{G_{1} / N}\left(\bar{\pi}_{N}^{G_{1}}\left(u_{\nu}\right)\right)$ holds. Here, since $G_{1} / N$ is a Lie group, the homomorphism $\Phi_{G_{1} / N}$ of $\boldsymbol{U}\left(G_{1} / N\right)$ into $\boldsymbol{D}_{l}\left(G_{1} / N\right)$ is an isomorphism onto. On the other hand, as is seen from the fact that $\left\{\bar{\pi}_{N}^{\left.G_{1}\left(\gamma_{i}\right) ; i \in J\right\}}\right.$ can be extended to a linear base of $R\left(G_{1} / N\right), \bar{\pi}_{N}^{G_{1}\left(u_{\nu}(k)\right)}$ $(k=1, \cdots, m)$ are linearly independent in $\boldsymbol{U}\left(G_{1} / N\right)$. Hence, after all, $\bar{D}_{\nu(k)}(k=$ $1, \cdots, m)$ are linearly independent in $\boldsymbol{D}_{l}\left(G_{1} / N\right)$. This means that $D_{\nu(k)}(k=1$, $\cdots, m)$ are linearly independent on $\mathscr{D}_{\infty}\left(G_{1}, N\right)$, hence a fortiori on $\mathscr{D}_{\infty}(G)$, which completes the proof. q.e.d.

Remark 2.1. From the above arguments, $\left\{D_{\nu} ; \nu \in \tilde{I}^{\prime}\right\}$ is an algebraic linear base of $D_{l}(G)$.

Proposition 2.1. Let $\alpha_{1}, \cdots, \alpha_{n}$ be any finite number of linearly independent elements of $R(G)$ which correspond to $X^{(1)}, \cdots, X^{(n)} \in L(G)$ respectively, and $\mu_{1}$, $\cdots, \mu_{n}$ be any positive integers. Put $D=D\left(\Pi_{i=1}^{n} \alpha_{i}^{\mu_{i}}\right)$. Then, for each $f \in \mathcal{E}_{\infty}(G)$ and $x \in G$, the function $f\left(x \exp _{G} \sum_{i=1}^{n} s_{i} X^{(i)}\right)$ in $\left(s_{1}, \cdots, s_{n}\right) \in \boldsymbol{R}^{n}$ is smooth (i.e., $\left.\in C^{\infty}\right)$ on some neighbourhood of $0 \in \boldsymbol{R}^{n}$, and there holds

$$
D f(x)=\left.\frac{\partial^{\Sigma \mu_{i}}}{\Pi \partial s_{i}^{\mu_{i}}} f\left(x \exp _{G} \sum_{i=1}^{n} s_{i} X^{(i)}\right)\right|_{\left(s_{i}\right)=(0)} .
$$

Proof. Evidently it suffices to consider the case $x=e$.

(I) First suppose that $G$ is finite-dimensional and Lie-projective. Take a totally disconnected $N \in \boldsymbol{H}_{0}(G)$ (Lemma 0.13). Then there exists a local homomorphism $\theta$ of $G$ into $N$ such that the map $x \mapsto\left(\pi_{N}^{G}(x), \theta(x)\right)$ sets up a local isomorphism of $G$ into $(G / N) \times N$ (Lemma 0.11). Therefore, for each $f \in \mathcal{E}_{\infty}(G)$, we have an $h \in \mathcal{E}_{\infty}((G / N) \times N)$ such that $f(x)=h\left(\pi_{N}^{G}(x), \theta(x)\right)$ holds for $x$ near e. Put $h_{1}=h(\cdot, \theta(e))\left(\in \mathcal{E}_{\infty}(G / N)\right)$. Then, since $\exp _{G}(L(G)) \cong c(G)$ and $\theta(c(G))=\{\theta(e)\}$, we have for small $s_{i}(i=1, \cdots, n)$,

$$
\begin{aligned}
f\left(\exp _{G} \sum_{i=1}^{n} s_{i} X^{(i)}\right) & =h_{1}\left(\pi_{N}^{G}\left(\exp _{G} \sum_{i=1}^{n} s_{i} X^{(i)}\right)\right) \\
& =h_{1}\left(\exp _{G / N} \sum_{i=1}^{n} s_{i} X_{N}^{(i)}\right) \quad(\text { by }(0.4)),
\end{aligned}
$$


where $X_{N}^{(i)}=\bar{\pi}_{N}^{G}\left(X^{(i)}\right)$. Since $G / N$ is a Lie group, this shows that the function $f\left(\exp _{G} \sum_{i=1}^{n} s_{i} X^{(i)}\right)$ in $\left(s_{i}\right)$ is certainly smooth in a neighbourhood of $0 \in \boldsymbol{R}^{n}$.

On the other hand, for each $\alpha \in R(G)$ and $x$ near $e$, we have

$$
d_{\alpha}^{(r)} f(x)=d_{(\bar{\alpha}, 0)}^{(r)} h\left(\pi_{N}^{G}(x), \theta(x)\right),
$$

where $\bar{\alpha}=\bar{\pi}_{N}^{G}(\alpha)$. Hence, obviously,

$$
D f(e)=\bar{D} h_{1}\left(\pi_{N}^{G}(e)\right),
$$

where $\bar{D}=D\left(\Pi_{i=1}^{n} \bar{\alpha}_{i}^{\mu}{ }_{i}\right)$. (Here $\bar{\alpha}_{i}(i=1, \cdots, n)$ are linearly independent in $R(G / N)$ since $N$ is totally disconnected (see (0.6)).) Since $G / N$ is a Lie group, the right side of (2.3) is given as $\left.\left(\partial^{\Sigma \mu_{i}} / \Pi \partial s_{i}^{\mu_{i}}\right) h_{1}\left(\exp _{G / N} \sum_{i=1}^{n} s_{i} X_{N}^{(i)}\right)\right|_{\left(s_{i}\right)=(0)}$, which equals $\left(\partial^{\Sigma \mu_{i}} / \Pi \partial s_{i}^{\mu_{i}}\right) f\left(\left.\exp _{G} \sum_{i=1}^{n} s_{i} X^{(i)}\right|_{\left(s_{i}\right)=(0)}\right.$ by (2.2). This completes the proof for the present case.

(II) Let $G$ be arbitrary. Take a pro-Lie open subgroup $G_{1}$ of $G$. Then, for each $f \in \mathcal{E}_{\infty}(G)$, we can choose $N \in \boldsymbol{H}\left(G_{1}\right)$ and $g \in D_{\infty}\left(G_{1} / N\right)$ so that $\bar{\alpha}_{i}=$ $\bar{\pi}_{N}^{G_{1}}\left(\alpha_{i}\right)(i=1, \cdots, n)$ are linearly independent in $R\left(G_{1} / N\right)$ (Lemma 2.5) and that $f=g \circ \pi_{N}^{G_{1}}$ holds on a neighbourhood of $e$ in $G_{1}$. Then, since $\exp _{G}\left(=\exp _{G_{1}}\right)$ maps $L(G)$ into $c(G)\left(\subseteq G_{1}\right)$, we have for small $s_{i}(i=1, \cdots, n)$,

$$
\begin{aligned}
f\left(\exp _{G} \sum_{i=1}^{n} s_{i} X^{(i)}\right) & =g\left(\pi_{N}^{G_{1}}\left(\exp _{G} \sum_{i=1}^{n} s_{i} X^{(i)}\right)\right) \\
& =g\left(\exp _{G_{1} / N} \sum_{i=1}^{n} s_{i} X_{N}^{(i)}\right),
\end{aligned}
$$

where $X_{N}^{(i)}=\bar{\pi}_{N}^{G_{1}}\left(X^{(i)}\right)$. Since $G_{1} / N$ is finite-dimensional, we see by $(I)$ this function in $\left(s_{i}\right)$ is smooth in a neighbourhood of $0 \in \boldsymbol{R}^{n}$. Besides, if we put $\bar{D}=D\left(\Pi_{i=1}^{n} \bar{\alpha}_{i}^{\mu_{i}}\right)$, then

$$
\begin{aligned}
D f(e) & =\bar{D} g\left(\pi_{N}^{G_{1}}(e)\right) \\
& =\left.\frac{\partial^{\Sigma \mu_{i}}}{\Pi \partial s_{i}^{\mu_{i}}} g\left(\exp _{G_{1} / N} \sum_{i=1}^{n} s_{i} X_{N}^{(i)}\right)\right|_{\left(s_{i}\right)=(0)} \\
& =\left.\frac{\partial^{\Sigma \mu_{i}}}{\Pi \partial s_{i}^{\mu_{i}}} f\left(\exp _{G} \sum_{i=1}^{n} s_{i} X^{(i)}\right)\right|_{\left(s_{i}\right)=(0)}
\end{aligned}
$$

The proof has now been completed. q.e.d.

The above formula (2.1) is well known if $G$ is a Lie group. It generalizes (1.1), and applies in particular to each $D_{\nu}\left(\nu \in \tilde{I}^{\prime}\right)$.

We conclude this section with simple remarks. Let $\theta$ be a continuous homomorphism of $G$ into another $L C$ group $G^{\prime}$. Then, evidently, the Lie algebra homomorphism $\bar{\theta}$ of $R(G)$ into $R\left(G^{\prime}\right)$ has a unique extension to an algebra homomorphism of $\boldsymbol{U}(G)$ into $\boldsymbol{U}\left(G^{\prime}\right)$ mapping 1 to 1 . In the sequel this extension will be denoted also by $\bar{\theta}$. It is easy to see that if $\bar{i} \bar{\theta}$ is injective or surjective as a map of $R(G)$ into $R\left(G^{\prime}\right)$, then so it is as a map of $\boldsymbol{U}(G)$ into $\boldsymbol{U}\left(G^{\prime}\right)$. If $\eta \circ \theta$ is a composition of group homomorphisms, then $\overline{\eta \circ \theta}=\bar{\eta} \circ \bar{\theta}$ holds as algebra homomorphism. In virtue of Theorem $2.2 \bar{\theta}$ can be viewed also as a homo- 
morphism of $\boldsymbol{D}_{l}(G)$ into $\boldsymbol{D}_{l}\left(G^{\prime}\right)$. For instance, if $N$ is a compact normal subgroup of $G, \bar{\pi}_{N}^{G}$ maps $\boldsymbol{D}_{l}(G)$ onto $\boldsymbol{D}_{l}(G / N)$. Here, if we identify $\mathscr{D}_{\infty}^{(*)}(G / N)$ with $\mathscr{D}_{\infty}^{(*)}(G, N), \bar{\pi}_{N}^{G}$ behaves as the restriction map $\left.D \in \boldsymbol{D}_{l}(G) \mapsto D\right|_{\mathscr{D}_{\infty}(G, N)}$.

2.4. Explicit form of differential operators. We now intend to describe all differential operators on $G$ explicitly. For this purpose we use a topological linear base of the minimal locally convex space $R(G)$ (see $\mathbf{0 . 1}$ ), taking after the discussions in [5], $\mathrm{n}^{\circ} 12$.

Let $\left\{\gamma_{i}\right\}_{i \in I}$ be a topological linear base of $R(G)$. We fix it for all subsequent arguments of the paper. Let $\left\{\gamma_{i}\right\}_{i \in I^{\prime}}\left(I \subseteq I^{\prime}\right)$ be an algebraic linear base of $R(G)$ extending $\left\{\gamma_{i}\right\}_{i \in I}$. Then, in view of the arguments in 2.3 , we have an algebraic linear base $\left\{D_{\nu} ; \nu \in \tilde{I}^{\prime}\right\}$ of $\boldsymbol{D}_{l}(G)$, where $\tilde{I}^{\prime}=\left\{\nu=\left(\nu_{i}\right)_{i \in I^{\prime}} \in Z_{+}^{I^{\prime}} ; \nu_{i}=0\right.$ for all but at most a finite number of $\left.i \in I^{\prime}\right\}$ and $D_{\nu}=D\left(\Pi_{\nu_{i} \neq 0 \gamma_{i}^{\nu} i}\right)\left(\nu \in \tilde{I}^{\prime}\right)$. For any subset $J$ of $I$, we put $\hat{J}=\left\{\nu \in \tilde{I}^{\prime} ; \nu_{i}=0\right.$ for all $\left.i \in I^{\prime} \backslash J\right\}$, and denote by $R_{J}(G)$ the closed linear subspace of $R(G)$ spanned by $\left\{\gamma_{i}\right\}_{i \in I \backslash J}$. Note that $\left\{D_{\nu} ; \nu \in \tilde{I}\right\}$ does not depend on the choice of $\gamma_{i}$ for $i \in I^{\prime} \backslash I$, and that one has $\left\{D_{\nu} ; \nu \in \tilde{I}\right\}=$ $\left\{D_{\nu} ; \nu \in \tilde{I}^{\prime}\right\}$ (i. e., $I=I^{\prime}$ ) only when $G$ is finite-dimensional.

Lemma 2.6. Suppose $G$ is Lie-projective. For each $N \in \boldsymbol{H}(G)$, there exists a finite subset $J$ of $I$ s.t. $R_{J}(G) \subseteq R(N)$.

Proof. Let $R(G)^{\prime}$ be the topological dual of $R(G)$. Since the topological dual of $\boldsymbol{R}^{I}$ is the restricted direct product $\boldsymbol{R}^{(I)}$, it is plain that $R(G)^{\prime}$ has an algebraic linear base $\left\{e_{i}\right\}_{i \in I}$ such that $e_{i}\left(\gamma_{j}\right)=1$ if $i=j$, and $=0$ otherwise $\left(i, j \in I\right.$ ). For a subset $M$ of $R(G)$ (resp. $\left.R(G)^{\prime}\right)$, let $M^{\perp}$ denote the annihilator of $M$ in $R(G)^{\prime}$ (resp. $R(G)$ ). Then, for $N \in \boldsymbol{H}(G), R(N)^{\perp}$ is fite-dimensional since it is the dual of the finite-dimensional quotient space $R(G) / R(N)(\cong R(G / N)$ by $(0,9))$. So we can choose a finite subset $J$ of $I$ such that the linear span of $\left\{e_{i} ; i \in J\right\}$ includes $R(N)^{\perp}$. Then, by the choice of $\left\{e_{i}\right\}_{i \in I}, R_{J}(G) \subseteq\left\{e_{i} ; i \in J\right\}^{\perp}$ $\subseteq R(N)^{\perp \perp}=R(N)$. $\quad$ q. e. d.

Corollary. Let $G_{1}$ be any pro-Lie open subgroup of $G$. For each $N \in \boldsymbol{H}\left(G_{1}\right)$, there exists a finite subset $J$ of $I$ such that $D_{\nu} f=0$ for all $\nu \in \tilde{I} \backslash \tilde{J}$ and $f \in \mathcal{E}_{\infty}(G, N)_{\rho}$.

Proof. Immediate from Lemmas 1.17 and 2.6. q.e.d.

Lemma 2.7. Let $I_{p}\left(\right.$ resp. $\left.I_{*}\right)$ denote the topology on $\overline{\boldsymbol{D}}(G)$ of uniform $\boldsymbol{\tau}_{p^{-}}$ convergence (resp. $\tau_{*}$-convergence) on each bounded subset of $\mathcal{E}_{\infty}^{(p)}(G)\left(\right.$ resp. $\left.\mathscr{D}_{\infty}^{(*)}(G)\right)$. Then $I_{p}$ and $I_{*}$ are equivalent.

Proof. Each bounded subset of $\mathscr{D}_{\infty}^{(*)}(G)$ is contained in $\mathscr{D}_{\infty}(G ; C)$ for some $C \in \operatorname{Com}(G)$, and $\tau_{*}$ coincides with $\tau_{p}$ there (Lemma 1.16). Besides, $\mathscr{D}_{\infty}(G ; C)$ is stable under every $D \in \overline{\boldsymbol{D}}(G)$. Hence, evidently, we have $\mathscr{I}_{p} \geqq I_{*}$. Next let $D^{(\lambda)}$ be any net in $\overline{\boldsymbol{D}}(G)$ converging to 0 in regard to $\mathscr{I}_{*}$. Take any bounded subset $\mathscr{B}$ of $\mathcal{E}_{\infty}^{(p)}(G)$ and $b \in \mathscr{D}_{\infty}(G)$. Choose $a \in \mathscr{D}(G)$ so that $a(x) \equiv 1$ on a neigh- 
bourhood of $\operatorname{supp}(b)$. Then $b D^{(\lambda)} f=b D^{(\lambda)}(a f)(f \in \mathscr{B})$. Since $a \mathscr{B}$ is bounded in $\mathscr{D}_{\infty}^{(*)}(G)$ and $b$ is arbitrary, this shows that the net $D^{(\lambda)} f$ converges to 0 in $\mathcal{E}_{\infty}^{(p)}(G)$ uniformly w.r.t. $f \in \mathscr{B}$. Hence $D^{(\lambda) \rightarrow 0}$ in regard to $\mathscr{I}_{p}$. Thus we have shown that $\mathscr{I}_{*} \geqq \mathscr{I}_{p}$, which completes the proof. q.e.d.

Definition 2.6. The equivalent topologies $\mathscr{I}_{p}$ and $\mathscr{I}_{*}$ on $\overline{\boldsymbol{D}}(G)$ are denoted by $\mathscr{I}$. $\overline{\boldsymbol{D}}(G)$ is equipped with $\mathscr{I}$ throughout. (Thus $\overline{\boldsymbol{D}}(G)$ is locally convex and Hausdorff.)

Definition 2.7. An element $\left(a_{\nu}\right)_{\nu \in I}$ of $\mathcal{E}_{\infty}(G)^{I}$, the set $\mathcal{E}_{\infty}(G)$ raised to the power $\tilde{I}$, is called admissible if for each finite subset $J$ of $I$ and $C \in \operatorname{Com}(G)$, the set $\left\{\nu \in \tilde{J} ; \operatorname{supp}\left(a_{\nu}\right) \cap C \neq \varnothing\right\}$ is at most finite.

Let $\left(a_{\nu}\right)_{\nu \in I}$ be an admissible element of $\mathcal{E}_{\infty}(G)^{I}$. Let $G_{1}$ be a pro-Lie open subgroup of $G$, and $(N, C) \in \boldsymbol{H}\left(G_{1}\right) \times \operatorname{Com}(G)$. Then, in virtue of Corollary to Lemma 2.6 and the admissibility of $\left(a_{\nu}\right)_{\nu \in \tilde{I}}$, all but at most a finite number of the differential operators $a_{\nu} D_{\nu}(\nu \in \tilde{I})$ vanish on $\mathscr{D}_{\infty}(G, N ; C)$. Hence it is clear that we can define a differential operator $D$ on $G$ as the map $f \in \mathscr{D}_{\infty}(G) \mapsto$ $\sum_{\nu \in I} a_{\nu} D_{\nu} f$. Now let $\boldsymbol{F}=\{F\}$ be the upper directed family of all finite subsets of $I$. Then, since each bounded subset of $\mathscr{D}_{\infty}^{(*)}(G)$ is contained in $\mathscr{D}_{\infty}(G, N ; C)$ for some $(N, C) \in \boldsymbol{H}\left(G_{1}\right) \times \operatorname{Com}(G)$, it is plain that the net $\left\{\sum_{\nu \in F} a_{\nu} D_{\nu} ; F \in \boldsymbol{F}\right\}$ in $\bar{D}(G)$ converges to $D$ in regard to $\mathscr{I}$. Thus we have

Lemma 2.8. For each admissible element $\left(a_{\nu}\right)_{\nu \in I}$ of $\mathcal{E}_{\infty}(G)^{\tilde{I}}$, the sum $\sum_{\nu \in I} a_{\nu} D_{\nu}$ of the differential operators $a_{\nu} D_{\nu}$ converges in the space $\overline{\boldsymbol{D}}(G)$ unorderedly.

Let $\left(a_{\nu}\right)_{\nu \in I}$ be as in Lemma 2.8. Put $D=\sum_{\nu \in \tilde{I}} a_{\nu} D_{\nu}$. Then, for each $f \in$ $\mathcal{E}_{\infty}(G)$, the numerical equality

$$
D f(x)=\sum_{\nu \in I} a_{\nu}(x) D_{\nu} f(x) \quad(x \in G)
$$

holds, where the right side reduces to a finite sum on each $C \in \operatorname{Com}(G)$. Indeed, from the definition of $\mathscr{I}, \sum_{\nu \in I} a_{\nu} D_{\nu} f$ converages to $D f$ in regard to $\boldsymbol{\tau}_{p}$, hence a fortiori pointwise. Here, if we choose $a \in \mathscr{D}(G)$ so that $a(x) \equiv 1$ on a neighbourhood of $C$, then $a_{\nu}(x) D_{\nu} f(x)=a_{\nu}(x) D_{\nu}(a f)(x)$ for $x \in C$. But, since $a f \in$ $\mathscr{D}_{\infty}(G, N$; $\operatorname{supp}(a))$ for some $N \in \boldsymbol{H}\left(G_{1}\right)$, the functions $a_{\nu} D_{\nu}(a f)$ vanish for all all but at most a finite number of $\nu$.

Lemma 2.8 enables us to associate to each admissible element $\left(a_{\nu}\right)_{\nu \in I}$ of $\mathcal{E}_{\infty}(G)^{I}$ a differential operator $D=\sum_{\nu \in I} a_{\nu} D_{\nu}$ on $G$. The explicit description of all differential operators on $G$ is now attained by the following theorem, which we prove in the next section.

Theorem 2.3. The map $\left(a_{\nu}\right)_{\nu \in I} \mapsto \sum_{\nu \in I} a_{\nu} D_{\nu}$ sets up a bijection of the set of all admissible elements of $\mathcal{E}_{\infty}(G)^{I}$ onto $\overline{\boldsymbol{D}}(G)$.

Remark 2.2. The following facts concerning the topology $\mathscr{I}$ will be used later on. (i) $\bar{D}(G)$ is a topological algebra under $\mathscr{I}$ (i.e., the multiplication is 
separately continuous). (ii) The algebra automorphism $D \mapsto \check{D}$ of $\overline{\boldsymbol{D}}(G)$ is topological. (iii) The injection $\alpha \mapsto d_{\alpha}^{(r)}$ of $R(G)$ into $\bar{D}(G)$ (Theorem 2.1) preserves the topology. (iv) $\boldsymbol{\delta}(G)$ and $\overline{\boldsymbol{D}}_{l}(G)$ are closed in $\overline{\boldsymbol{D}}(G)$.

Let us check (iii). Since the space $R(G)$ is of minimal type, it suffices to see that the above injection is continuous. Let $\left\{\alpha^{(\lambda)}\right\}$ be a net in $R(G)$ converging to 0 , and $\mathcal{B}$ a bounded subset of $\mathscr{D}_{\infty}^{(*)}(G)$. Our job is to show that $d_{\alpha(\lambda)}^{(r)} f$ converges to 0 in $\mathscr{D}_{\infty}^{(*)}(G)$ uniformly w.r.t. $f \in \mathscr{B}$. Take a pro-Lie open subgroup $G_{1}$ of $G$. Then $\mathscr{B}$ is a bounded subset of $\mathscr{D}_{\infty}^{(r)}(G, N ; C)_{\rho}$ for some $(N, C) \in \boldsymbol{H}\left(G_{1}\right) \times \operatorname{Com}(G)$. Choose a finite number of $\beta_{1}, \cdots, \beta_{n} \in R(G)$ so that $\bar{\beta}_{k}=\bar{\pi}_{N}^{G_{1}}\left(\beta_{k}\right) \quad(k=1, \cdots, n)$ constitute a linear base of $R\left(G_{1} / N\right)$. Then each $\bar{\pi}_{N}^{G_{1}}\left(\alpha^{(\lambda)}\right)$ is expressed as $\sum_{k=1}^{n} c_{k}^{(\lambda)} \bar{\beta}_{k}\left(c_{k}^{(\lambda)} \in \boldsymbol{R}\right)$. Here $c_{k}^{(\lambda)} \rightarrow 0$ for all $k$ since $\bar{\pi}_{N}^{G_{1}}\left(\alpha^{(\lambda)}\right) \rightarrow 0$. Meanwhile, each $f \in \mathscr{D}_{\infty}(G, N)_{\rho}$ is expressed as a finite sum of left translations of functions in $\mathscr{D}_{\infty}\left(G, N ; G_{1}\right)_{\rho}\left(\cong \mathscr{D}_{\infty}\left(G_{1}, N\right)\right)$. Hence, in view of (1.3), $\quad d_{\alpha}^{(r)} f=\sum_{k=1}^{n} c_{k}^{(\lambda)} d_{\beta}^{(r)} f$ holds for $f \in \mathscr{D}_{\infty}(G, N)_{\rho}$. Therefore it is now plain that $d_{\alpha}^{(r)} f(\lambda) f$ converges to 0 in $\mathscr{D}_{\infty}^{(r)}(G, N ; C)_{\rho}$ (hence in $\mathscr{D}_{\infty}^{(*)}(G)$ ) uniformly w. r. t. $f \in \mathscr{B}$.

2.5. Proof of Theorem 2.3. We retain the notations as in 2.4.

Lemma 2.9. Suppose $G$ is Lie-projective. For any finite subset $J$ of $I$, there exists an $N \in \boldsymbol{H}_{0}(G)$ s.t. $R(N) \cong R_{J}(G)$.

Proof. Put $\mathcal{L}=R_{I \backslash J}(G)$. Then $\mathcal{L}$ and $R_{J}(G)$ are the topological complements of each other in $R(G)$. Let $P_{\mathcal{L}}$ denote the projection of $R(G)$ onto $\mathcal{L}$. Since $\mathcal{L}$ is finite-dimensional, we can choose a neighbourhood $U$ of 0 in $\mathcal{L}$ including no linnear subspace other than $\{0\}$. Now, as a neighbourhood of 0 in $R(G), P_{\perp}^{-1}(U)$ includes $R(N)$ (=ker $\left(d \pi_{N}^{G}\right)$ ) for some $N \in \boldsymbol{H}_{0}(G)$. Then, by the choice of $\mathcal{U}, R(N) \subseteq R_{J}(G)$, which completes the proof. q. e. d.

Now let us denote by $X^{(i)}$ the element of $L(G)$ corresponding to $\gamma_{i}(i \in I)$. Since the map $\left(s_{i}\right)_{i \in I} \in \boldsymbol{R}^{I} \mapsto \sum_{i \in I} s_{i} X^{(i)}$ is a topological linear isomorphism of $\boldsymbol{R}^{I}$ onto $L(G)$, we have, for each $f \in \mathscr{D}(G)$ and $x \in G$, a function $\left(s_{i}\right)_{i \in I} \mapsto$ $f\left(x \exp _{G} \sum_{i \in I} s_{i} X^{(i)}\right)$ defined on $\boldsymbol{R}^{I}$.

Note. In reality the above function depends only on a finite number of $s_{i}$. Indeed, take a pro-Lie open subgroup $G_{1}$ of $G$. Then, since ${ }_{x} f \in \mathscr{D}_{\infty}(G, N)_{\rho}$ for some $N \in \boldsymbol{H}_{0}\left(G_{1}\right)$, one has $g \in \mathscr{D}\left(G_{1} / N\right)$ such that ${ }_{x} f=g \circ \pi_{N^{1}}^{G_{1}}$ holds on $G_{1}$. Since $\exp _{G}\left(=\exp _{G_{1}}\right)$ maps $\sum_{i \in I} s_{i} X^{(i)}\left(\left(s_{i}\right) \in \boldsymbol{R}^{I}\right)$ into $c(G)\left(\subseteq G_{1}\right)$, we have

$$
\begin{aligned}
{ }_{x} f\left(\exp _{G} \sum_{i \in I} s_{i} X^{(i)}\right) & =g\left(\pi_{N^{1}}^{G_{1}}\left(\exp _{G} \sum_{i \in I} s_{i} X^{(i)}\right)\right) \\
= & g\left(\exp _{G_{1} / N} \sum_{i \in I} s_{i} X_{N}^{(i)}\right) \quad(\text { by }(0.4)),
\end{aligned}
$$

where $X_{N}^{(i)}=\bar{\pi}_{N}^{G_{1}}\left(X^{(i)}\right)$. Here, by Lemma 2.6, there exists a finite subset $J$ of $I$ such that $R_{J}\left(G_{1}\right) \subseteq R(N)$, i.e., that $X_{N}^{(i)}=0$ for all $i \in I \backslash J$. Thus ${ }_{x} f\left(\exp _{G} \sum_{i \in I} s_{i} X^{(i)}\right)$ depends only on $s_{i}(i \in J)$. 
Lemma 2.10. Let $G_{1}$ be a pro-Lie open subgroup of $G$. Let $\nu=\left(\nu_{i}\right)_{i \in I} \in \tilde{I}$ and $x \in G$. Put $J=\left\{i \in I ; \nu_{i} \neq 0\right\}$ and choose an $N \in \boldsymbol{H}_{0}\left(G_{1}\right)$ s.t. $R(N) \subseteq R_{J}\left(G_{1}\right)$ $\left(=R_{J}(G)\right.$ ) (Lemma 2.9). Then there exists a function $f \in \mathscr{D}_{\infty}(G, N)_{\rho}$ such that

$$
f\left(x \exp _{G} \sum_{i \in I} s_{i} X^{(i)}\right)=\Pi_{i \in J}\left(\nu_{i} !\right)^{-1} s_{i}^{\nu i}
$$

holds on a neighbourhood of 0 in $\boldsymbol{R}^{I}$.

Proof. It suffices to consider the case $x=e$. So, moreover, we can assume that $G$ is Lie-projective and $G_{1}=G$. For any finite subset $J_{1}$ of $I$, let $L_{J_{1}}(G)$ denote the closed linear subspace of $L(G)$ corresponding to $R_{J_{1}}(G)$. For any $X \in L(G)$ and $N_{1} \in H_{0}(G)$, put $X_{N_{1}}=\bar{\pi}_{N_{1}}^{G}(X)$.

Now let us choose by Lemmas 2.6 and 2.9 a finite subset $J^{\prime}$ of $I$ and $N^{\prime} \in$ $\boldsymbol{H}_{0}(G)$ so that $R_{N^{\prime}}(G) \subseteq R_{J^{\prime}}(G) \subseteq L_{N}(G)$ and $N^{\prime} \subseteq N$. Then, since $L_{N}(G) \subseteq L_{J}(G)$ by the assumption of the lemma, we have $J \subseteq J^{\prime}$. It is evident that $\left\{X_{N}^{(i)} ; i \in I\right\}$ (resp. $\left.\left\{X_{N^{\prime}}^{(i)} ; i \in I\right\}\right)$ spans $L(G / N)$ (resp. $L\left(G / N^{\prime}\right)$ ) algebraically. This together with the inclusion relation $L_{N^{\prime}}(G) \cong L_{N}(G) \cong L_{J}(G)$ enables us to choose a finite number of $Y^{(j)} \in L_{J}(G)$ and $Z^{(k)} \in L_{N}(G)$ so that $X_{N}^{(i)}(i \in J)$ together with $Y_{N}^{(j)}$ constitute a linear base of $L(G / N)$, and $X_{N^{\prime}}^{(i)}(i \in J)$ together with $Y_{N^{\prime}}^{(j)}$ and $Z_{N^{\prime}}^{(k)}$ constitute that of $L\left(G / N^{\prime}\right)$. On the other hand, since $L_{N^{\prime}}(G) \subseteq L_{J^{\prime}}(G)$, we can choose a finite number of $W^{(l)} \in L_{J^{\prime}}(G)$ so that $X_{N^{\prime}}^{(2)}\left(i \in J^{\prime}\right)$ together with $W_{N^{\prime}}^{(l)}$ constitute a linear base of $L\left(G / N^{\prime}\right)$. Note that the change between the bases $\left\{X_{N^{\prime}}^{(i)}(i \in J), Y_{N^{\prime}}^{(j)}, Z_{N^{\prime}}^{(k)}\right\}$ and $\left\{X_{N^{\prime}}^{(i)}(i \in J), X_{N^{\prime}}^{(i)}\left(i \in J^{\prime} \backslash J\right), W_{N^{\prime}}^{(i)}\right\}$ of $L\left(G / N^{\prime}\right)$ is performed by the transformation

$$
\left(\begin{array}{ll}
1_{J} & 0 \\
0 & *
\end{array}\right)
$$

where $1_{J}$ denotes the identity matrix of order $|J|$, the cardinal of $J$.

Since $G / N$ is a Lie group, there exists a $g \in \mathscr{D}(G / N)$ such that

$$
g\left(\exp _{G / N}\left(\sum_{i \in J} s_{i} X_{N}^{(i)}+\sum_{j} t_{j} Y_{N}^{(j)}\right)\right)=\prod_{i \in J}(\nu !)^{-1} s_{i}^{\iota} i
$$

holds so long as the real parameters $s_{i}$ and $t_{j}$ are sufficiently small. Put $f=$ $g \circ \pi_{N}^{G}\left(\in \mathscr{D}_{\infty}(G, N)\right)$. Since $N^{\prime} \subseteq N$, there exists $g^{\prime} \in \mathscr{D}\left(G / N^{\prime}\right)$ s.t. $f=g^{\prime} \circ \pi_{N^{\prime}}^{G}$. Now, since $Z_{N}^{(k)}=0$ for all $k$, the left side of (2.5) equals $g\left(\exp _{G / N}\left(\sum_{i \in J} s_{i} X_{N}^{(i)}+\right.\right.$ $\left.\left.\sum_{j} t_{j} Y_{N}^{(j)}+\sum_{k} u_{k} Z_{N}^{(k)}\right)\right)$ for any $u_{k} \in \boldsymbol{R}$. By (0.4) and through the above change of the bases, this is furthermore equal to

$$
\begin{aligned}
& f\left(\exp _{G}\left(\sum_{i \in J} s_{i} X^{(i)}+\sum_{j} t_{j} Y^{(j)}+\sum_{k} u_{k} Z^{(k)}\right)\right) \\
= & g^{\prime}\left(\exp _{G / N^{\prime}}\left(\sum_{i \in J} s_{i} X_{N^{\prime}}^{(i)}+\sum_{j} t_{j} Y_{N^{\prime}}^{(j)}+\sum_{k} u_{k} Z_{N^{\prime}}^{(k)}\right)\right) \\
= & g^{\prime}\left(\exp _{G / N^{\prime}}\left(\sum_{i \in J} s_{i} X_{N^{\prime}}^{(i)}+\sum_{i \in J^{\prime} \backslash J} s_{i} X_{N^{\prime}}^{(i)}+\sum_{l} v_{l} W_{N^{\prime}}^{(l)}\right)\right),
\end{aligned}
$$

where $s_{i}\left(i \in J^{\prime} \backslash J\right)$ and $v_{l}$ are connected with $t_{j}$ and $u_{k}$ through the transformation $*$ appearing in the above matrix. Thus, after all, we have

$$
g^{\prime}\left(\exp _{G / N^{\prime}}\left(\sum_{i \in J^{\prime}} s_{i} X_{N^{\prime}}^{(i)}+\sum_{l} v_{l} W_{N^{\prime}}^{(l)}\right)\right)=\prod_{i \in J}\left(\nu_{i} !\right)^{-1} s_{i}^{\imath^{i}},
$$

provided the parameters $s_{i}$ and $v_{l}$ are sufficiently small. Since this does not 
depend on $v_{l}$, we can put here $v_{l}=0$. Then

$$
f\left(\exp _{G} \sum_{i \in J^{\prime}} s_{i} X^{(i)}\right)=\prod_{i \in J}\left(\nu_{i} !\right)^{-1} s_{i}^{\nu} i,
$$

$s_{i}\left(i \in J^{\prime}\right)$ being supposed to be small. Here, since $L_{J^{\prime}}(G) \leqq L_{N}(G)$ and so $X_{N}^{(i)}=0$ for $i \in I \backslash J^{\prime}$, the left side is equal to

$$
\begin{aligned}
g\left(\exp _{G / N} \sum_{i \in J^{\prime}} s_{i} X_{N}^{(i)}\right) & =g\left(\exp _{G / N} \sum_{i \in I} s_{i} X_{N}^{(i)}\right) \\
& =f\left(\exp _{G} \sum_{i \in I} s_{i} X^{(i)}\right)
\end{aligned}
$$

for any $s_{i} \in \boldsymbol{R}\left(i \in I \backslash J^{\prime}\right)$. The proof is now complete. q.e.d.

Corollary. For each $\nu \in \tilde{I}$ and $x \in G$, there exists a function $f \in \mathscr{D}(G)$ s.t.

$$
D_{\nu^{\prime}} f(x)= \begin{cases}1 & \text { if } \nu^{\prime}=\nu, \\ 0 & \text { otherwise (i.e., } \left.\nu^{\prime} \in \tilde{I}, \neq \nu\right) .\end{cases}
$$

Such an $f$ can be chosen in $\mathscr{D}_{\infty}(G, N)_{\rho}$ if $N$ is taken as in Lemma 2.10.

Proof. Using Proposition 2.1, we see easily that the function $f$ given in Lemma 2.10 satisfies (2.6). q.e.d.

Proof of Theorem 2.3. Let $D \in \overline{\boldsymbol{D}}(G)$. Our task is to show that there exists a unique admissible element $\left(a_{\nu}\right)_{\nu \in I}$ of $\mathcal{E}_{\infty}(G)^{I}$ for which $D=\sum_{\nu \in I} a_{\nu} D_{\nu}$ holds. But, in view of (2.4) and Corollary to Lemma 2.10, the uniqueness is obvious. So we prove its existence.

(I) First suppose $G$ is Lie-projective. For this case we make the proof in three steps. $\left(I_{a}\right)$ Let $N \in \boldsymbol{H}_{0}(G)$ and $C \in \operatorname{Com}(G)$. For each $x \in G$, consider a distribution $T(x)$ on the Lie group $G / N$ defined by $\langle g, T(x)\rangle=D\left(g \circ \pi_{N}^{G}\right)(x)$ $(g \in \mathscr{D}(G / N))$. Then, since $\operatorname{supp}(T(x)) \subseteq\left\{\pi_{N}^{G}(x)\right\}$, there exists a unique element $D_{N}(x) \in D_{l}(G / N)$ s.t.

$$
D\left(g \circ \pi_{N}^{G}\right)(x)=\left(D_{N}(x) g\right)\left(\pi_{N}^{G}(x)\right) \quad(g \in \mathscr{D}(G / N)) .
$$

We now show that the orders of $D_{N}(x)(x \in C)$ (what is the same, the orders of $T(x)(x \in C))$ are bounded.

To do so, let us choose $a \in \mathscr{D}_{\infty}(G, N)$ s.t. $a(x) \equiv 1$ on a neighbourhood of $C$. Since the map $f \in \mathscr{D}_{\infty}^{(r)}(G, N ; \operatorname{supp}(a)) \mapsto D f \in \mathcal{E}_{0}(G)$ is continuous, we can choose a finite number of $D_{1}, \cdots, D_{p} \in D_{l}(G)$ so that

$$
\sup _{x \in C}|D(a f)(x)| \leqq \sum_{i=1}^{p} \sup _{y \in G}\left|D_{i}(a f)(y)\right| \quad\left(f \in \mathscr{D}_{\infty}(G, N)\right) .
$$

If a finite number of $D_{1}^{\prime}, \cdots, D_{q}^{\prime} \in \boldsymbol{D}_{l}(G)$ are suitably chosen, the right side of this is majorized by $\sum_{i=1}^{q} \sup _{y \in G}\left|D_{i}^{\prime} f(y)\right|$. On the other hand, by the choice of $a, D(a f)(x)$ $=D f(x)$ holds for any $f \in \mathcal{E}_{\infty}(G)$ and $x \in C$. Thus we have

$$
\sup _{x \in C}|D f(x)| \leqq \sum_{i=1}^{q} \sup _{y \in G}\left|D_{i}^{\prime} f(y)\right| \quad\left(f \in \mathscr{D}_{\infty}(G, N)\right) .
$$

This can be rewritten as 


$$
\sup _{x \in C}\left|D\left(g \circ \pi_{N}^{G}\right)(x)\right| \leqq \sum_{i=1}^{q} \sup _{y \in G}\left|\bar{D}_{i}^{\prime} g\left(\pi_{N}^{G}(y)\right)\right| \quad(g \in \mathscr{D}(G / N)),
$$

where $\bar{D}_{i}^{\prime}=\bar{\pi}_{N}^{G}\left(D_{i}^{\prime}\right)$ (see the end of 2.3). The last inequality shows that the orders $T(x)(x \in C)$ are certainly bounded.

$\left(\mathrm{I}_{b}\right) \quad$ Let $J$ be any finite subset of $I$. Choose an $N \in \boldsymbol{H}_{0}(G)$ s.t. $R(N) \subseteq R_{J}(G)$ (Lemma 2.9). And choose a finite subset $J^{\prime}$ of $I$ so that $\bar{\pi}_{N}^{G}\left(\gamma_{i}\right)\left(i \in J^{\prime}\right)$ consititute a linear base of $R(G / N)$. This is possible since $\bar{\pi}_{N}^{G}\left(\gamma_{i}\right)(i \in I)$ span $R(G / N)$. Note that these choices imply $J \subseteq J^{\prime}$. We now intend to apply the result in $\left(I_{a}\right)$ to the present $N$. Evidently $\bar{D}_{\nu}=\bar{\pi}_{N}^{G}\left(D_{\nu}\right) \quad\left(\nu \in \tilde{J}^{\prime}\right.$ constitute an algebraic linear base of $\boldsymbol{D}_{l}(G / N)$. Therefore, for each $x \in G, D_{N}(x)$ is expressed uniquely as $D_{N}(x)=\sum_{\nu \in J^{\prime}} a_{N \nu}(x) \bar{D}_{\nu}$, where all but at most a finite number of $a_{N \nu}(x)(\in \boldsymbol{C})$ are 0 . Thus (2.7) has now the form

$$
D\left(g \circ \pi_{N}^{G}\right)(x)=\sum_{\nu \in \tilde{J}^{\prime}} a_{N \nu}(x) \bar{D}_{\nu} g\left(\pi_{N}^{G}(x)\right) \quad(g \in \mathscr{D}(G / N)),
$$

which is rewritten as

$$
D f(x)=\sum_{\nu \in J^{\prime}} a_{N_{\nu}}(x) D_{\nu} f(x) \quad\left(f \in \mathscr{D}_{\infty}(G, N)\right) .
$$

Let us now consider the functions $a_{N \nu}: x \mapsto a_{N \nu}(x)$ defined on $G\left(\nu \in \tilde{J}^{\prime}\right)$. Let $O$ be any relatively compact open neighbourhood of a point $x_{0} \in G$. Then, by $\left(I_{a}\right)$, the orders of $D_{N}(x)(x \in O)$ are bounded, and therefore, all except at most a finite number of $a_{N \nu}$ vanish on $O$. Let $\left\{a_{N_{\nu}(1)}, \cdots, a_{N_{\nu}(p)}\right\}$ be the totality of those exceptional functions. By Corollary to Lemma 2.10 we can choose $f_{j} \in \mathscr{D}(G)$ $(j=1, \cdots, p)$ so that $D_{\nu(i)} f_{j}\left(x_{0}\right)=1$ if $i=j$, and $=0$ otherwise. Then, from (2.8), we have

$$
\left(D f_{1}(x), \cdots, D f_{p}(x)\right)=\left(a_{N_{\nu}(1)}(x), \cdots, a_{N_{\nu}(p)}(x)\right) A(x) \quad(x \in O),
$$

where $A(x)$ denotes a matrix of $\operatorname{order} p$ with $(i, j)$-component $D_{2}(i) f_{j}(x)$. If $O$ is taken to be sufficiently small, $A(x)$ is non-singular for every $x \in O$ because $A\left(x_{0}\right)=1$, an identity matrix. Therefore, from (2.9), we see that all $a_{N_{\nu}(i)}$ are smooth on $O$ (i.e., "indefinitely continuously differentiable on $O$ "). Since $x_{0}$ is arbitrary, we have thus seen that all $a_{N_{\nu}}\left(\nu \in \tilde{J}^{\prime}\right)$ belong to $\mathcal{E}_{\infty}(G)$ and also that they form a locally finite family (i.e., the set $\left\{\nu \in \tilde{J}^{\prime} ; \operatorname{supp}\left(a_{N_{\nu}}\right) \cap C \neq \varnothing\right\}$ is at most finite for every $C \in \operatorname{Com}(G)$ ).

(2.8) together with Corollary to Lemma 2.10 obviously shows that each of $a_{N \nu}$ for $\nu \in \tilde{J}$ is determined only by $\nu$, not depending on the special choice of $N$ and $J^{\prime}$ in the above indicated manner. So, for $\nu \in \tilde{J}$, let us write $a_{\nu}$ instead of $a_{N \nu}$. Also let us write (2.8) as

$$
D f(x)=\sum_{\nu \in J} a_{\nu}(x) D_{\nu} f(x)+\sum_{\nu \in J^{\prime} \backslash J \tilde{J}} a_{N_{\nu}}(x) D_{\nu} f(x) \quad\left(f \in \mathscr{D}_{\infty}(G, N), x \in G\right) .
$$

$\left(I_{c}\right)$ The procedure in $\left(I_{b}\right)$ yields, for every finite subset $J$ of $I$, a locally finite subfamily $\left\{a_{\nu} ; \nu \in \tilde{J}\right\}$ of $\mathcal{E}_{\infty}(G)$. Here, as is seen from what was just mentioned, each $a_{\nu}$ is determined not depending on the special choice of $J$ s.t. $\nu \in \tilde{J}$. Thus, after all, we obtain an admissible element $\left(a_{\nu}\right)_{\nu \in \tilde{I}}$ of $\mathcal{E}_{\infty}(G)^{\tilde{I}}$. We now show that $D=\sum_{\nu \in I} a_{\nu} D_{\nu}$ holds. To do so it suffices to show that for 
each $f \in \mathscr{D}(G)$ and $x \in G, D f(x)=\sum_{\nu \in I} a_{\nu}(x) D_{\nu} f(x)$ holds. Choose an $N_{1} \in H_{0}(G)$ and a finite subset $J$ of $I$ so that $f \in \mathscr{D}_{\infty}\left(G, N_{1}\right)$ and $R_{J}(G) \subseteq R\left(N_{1}\right)$ (Lemma 2.6). Then, since $d_{\alpha}^{(r)} h=0$ for any $h \in \mathscr{D}_{\infty}\left(G, N_{1}\right)$ and $\alpha \in R_{J}(G)$, we have

$$
D_{\nu} f=0 \quad(\nu \in \tilde{I} \backslash \tilde{J}) .
$$

Next let us choose an $N \in \boldsymbol{H}_{0}(G)$ and a finite subset $J^{\prime}$ of $I$ so that $N \cong N_{1}$ (hence $f \in \mathscr{D}_{\infty}(G, N)$ ), $R(N) \subseteq R_{J}(G)$, and $\bar{\pi}_{N}^{G}\left(\gamma_{i}\right)\left(i \in J^{\prime}\right)$ constitute a linear base of $R(G / N)$. For the present $J, N, J^{\prime}$ and $f,(2.10)$ is of course valid. Therefore, by (2.11), we see that the equality $D f(x)=\sum_{\nu \in I} a_{\nu}(x) D_{\nu} f(x)$ holds. The proof for the Lie-projective case has thus been completed.

(II) Next let $G$ be arbitrary. Take a pro-Lie open subgroup $G_{1}$ of $G$. Let us recall Remark 1.3 and denote by $D_{\lambda}$ the restriction of $D$ to $D_{\infty}\left(G ; \Gamma_{\lambda}\right)$. Then the map $g \in \mathscr{D}_{\infty}\left(G_{1}\right) \mapsto_{x_{\lambda}}\left(D_{\lambda}\left({ }_{x} \bar{\lambda}^{-1} g\right)\right)$ belongs to $\overline{\boldsymbol{D}}\left(G_{1}\right)$. Therefore, by (I), there exists an admissible element $\left(a_{\nu}^{(\lambda)}\right)_{\nu \in I}$ of $\mathcal{E}_{\infty}\left(G_{1}\right)^{I}$ such that

$$
{ }_{x_{\lambda}}\left(D_{\lambda}\left({ }_{x \bar{\lambda}^{1}} g\right)\right)(x)=\sum_{\nu \in I} a_{\nu}^{(\lambda)}(x) D_{\nu} g(x) \quad\left(g \in \mathscr{D}_{\infty}\left(G_{1}\right), x \in G_{1}\right) .
$$

Hence

$$
D_{\lambda} h(x)=\sum_{\nu \in I} a_{\nu}^{(\lambda)}\left(x_{\lambda}^{-1} x\right) D_{\nu} h(x) \quad\left(h \in \mathscr{D}_{\infty}\left(G ; \Gamma_{\lambda}\right), x \in \Gamma_{\lambda}\right) .
$$

For each $\nu \in \tilde{I}$, define a function $a_{\nu} \in \mathcal{E}_{\infty}(G)$ as $a_{\nu}=\bigcup_{\lambda} x_{\bar{\lambda}^{-1}} a_{\nu}^{(\lambda)}$. Then $\left(a_{\nu}\right)_{\nu \in \tilde{I}}$ $\left(\in \mathcal{E}_{\infty}(G)^{I}\right)$ is evidently admissible. Besides, from (2.12),

$$
D f(x)=\sum_{\nu \in I} a_{\nu}(x) D_{\nu} f(x) \quad\left(f \in \mathscr{D}_{\infty}(G), x \in G\right) .
$$

That is, $D=\sum_{\nu \in I} a_{\nu} D_{\nu}$ holds. The proof of the theorem is now complete. q.e.d.

2.6. Elements of $\boldsymbol{\delta}(G)$ and $\overline{\boldsymbol{D}}_{l}(G)$. In this section we determine, among all differential operators on $G$, the elements of $\boldsymbol{\delta}(G)$ and $\overline{\boldsymbol{D}}_{l}(G)$.

Lemma 2.11. Let $D=\sum_{\nu \in I} a_{\nu} D_{\nu}$, where $\left(a_{\nu}\right)_{\nu \in I}$ is an admissible element of $\mathcal{E}_{\infty}(G)^{1}$. For $D$ to be a derivation (i.e., $\in \boldsymbol{\delta}(G)$ ), it is necessry and sufficient that $a_{\nu}=0$ unless $|\nu|=1$, where $|\nu|=\sum_{i \in I} \nu_{i}$.

Proof. If $|\nu|=1, D_{\nu}$ coincides with $d_{r_{i}}^{(r)}$ for some $i \in I$. Hence the sufficiency of the condition is obvious (see Remark 2.2, (iv)). Conversely suppose that $D$ is a derivation. For each $\nu^{(0)} \in \tilde{I}$ with $\left|\nu^{(0)}\right|>1$, we can choose $\nu^{\prime}, \nu^{\prime \prime} \in \tilde{I}$ so that $\left|\nu^{\prime}\right|>1,\left|\nu^{\prime \prime}\right|>1$ and $\nu_{i}^{\prime}+\nu_{i}^{\prime \prime}=\nu_{i}^{(0)}$ for all $i \in I$. For each $x \in G$, choose $f, g \in \mathscr{D}(G)$ such that $f\left(x \exp _{G} \sum_{i \in I} s_{i} X^{(i)}\right)=\prod_{\nu_{i}^{\prime} \neq 0} s_{i}^{\nu^{\prime} i}$ and $g\left(x \exp _{G} \sum_{i \in I} s_{i} X^{(i)}\right)=\prod_{\nu_{i}^{\prime \prime} \neq 0} s_{i}^{\nu^{\prime \prime} i}$ hold on some neighbourhood $O$ of 0 in $\boldsymbol{R}^{I}$ (Lemma 2.10.). Put $h=f g$. Then $h\left(x \exp _{G} \sum_{i \in I} s_{i} X^{(i)}\right)=\Pi_{\nu_{i}^{(0)} \neq 0} s_{i}^{\iota^{(0)}}$ on $O$. Hence, by Proposition 2.1, $D h(x)=$

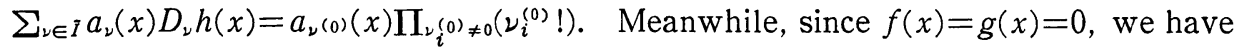
$D h(x)=(D f(x)) g(x)+f(x) D g(x)=0$. Thus, after all, $a_{\nu(0)}(x)=0$. Since $\nu^{(0)}$ and $x$ are arbitrary, it has been shown that $a_{\nu}=0$ if $|\nu|>1$. Now, if $|\nu|=0$, then $a_{\nu}=D(1)$. Since $D$ is a derivation, we have $D(1)=0$. The necessity of our condition has thus been proved. q.e.d. 
It is plain that there exists a natural bijection of $\mathcal{E}_{\infty}(G)^{I}$ onto the set of all admissible elements of $\mathcal{E}_{\infty}(G)^{I}$ which satisfy the condition stated in Lemma 2.11. So the following proposition holds.

Proposition 2.2. For each $\left(a_{i}\right)_{i \in I} \in \mathcal{E}_{\infty}(G)^{I}$, the sum $\sum_{i \in I} a_{i} d_{r_{i}}^{(r)}$ of the elements $a_{i} d_{r_{i}}^{(r)}$ of $\boldsymbol{\delta}(G)$ converges in the space $\overline{\boldsymbol{D}}(G)$ unoderedly. The map $\left(a_{i}\right)_{i \in I} \mapsto$ $\sum_{i \in I} a_{i} d_{r_{i}}^{(r)}$ sets up a bijection of $\mathcal{E}_{\infty}(G)^{I}$ onto $\boldsymbol{\delta}(G)$.

Lemma 2.12. Let $D=\sum_{\nu \in I} a_{\nu} D_{\nu}$, where $\left(a_{\nu}\right)_{\nu \in I}$ is an admissible element of $\mathcal{E}_{\infty}(G)^{\tilde{I}}$. For $D$ to be left invariant (i.e., $\in \overline{\boldsymbol{D}}_{l}(G)$ ), it is necessary and sufficient that all functions $a_{\nu}$ are constant.

Proof. $D$ is left invariant if and only if we have $D f(x)=D\left({ }_{x} f\right)(e)$, i. e., $\sum_{: \in I} a_{\nu}(x) D_{\nu} f(x)=\sum_{\nu \in I} a_{\nu}(e) D_{\nu} f(x)$, for all $f \in \mathcal{E}_{\infty}(G)$ and $x \in G$. In view of Corollary to Lemma 2.10, this is equivalent to that $a_{2}(x)=a_{2}(e)$ holds for all $\nu \in \tilde{I}$ and $x \in G$, i. e., that all $a_{2}$ are constant. q.e.d.

Now let us call an element $\left(c_{\nu}\right)_{2 \in \tilde{I}}$ of $\boldsymbol{C}^{\tilde{I}}$ admissible if, for each finite subset $J$ of $I$, the set $\left\{\nu \in \tilde{J} ; c_{\nu} \neq 0\right\}$ is at most finite. If we view each $c_{\nu}$ as a constant function on $G$, this condition is equivalent to that $\left(c_{\nu}\right)_{\nu \in I}$ is an admissible element of $\mathcal{E}_{\infty}(G)^{\tilde{I}}$. Therefore, by Lemma 2.12 , the following proposition holds.

Proposition 2.3. For each admissible element $\left(c_{\nu}\right)_{\nu \in I}$ of $\boldsymbol{C}^{\tilde{I}}$, the sum $\sum_{\nu \in I} c_{\nu} D_{\nu}$ of the elements $c_{\nu} D_{\nu}$ of $\boldsymbol{D}_{l}(G)$ converges in the space $\overline{\boldsymbol{D}}(G)$ unorderedly. The map $\left(c_{\nu}\right)_{\nu \in I^{I}} \mapsto \sum_{\nu \in I} C_{\nu} D_{\nu}$ sets up a bijection of the set of all admissible elements of $\boldsymbol{C}^{I}$ onto $\overline{\boldsymbol{D}}_{l}(G)$.

Corollary 1. $\boldsymbol{D}_{l}(G)$ is $\mathcal{I}$-dense in $\overline{\boldsymbol{D}}_{l}(G)$, and coincides with it if $G$ is finitedimensional.

Proof. The density in question is clear from Proposition 2.3. Now suppose $G$ is finite-dimensional. Then the set $I$ is finite. Therefore each admissible element $\left(c_{\nu}\right)_{\nu \in I}$ of $\boldsymbol{C}^{\bar{I}}$ has at most a finite number of non-zero components. Hence, by Proposition 2.3, $\overline{\boldsymbol{D}}_{l}(G)=\boldsymbol{D}_{l}(G)$. q. e. d.

In 2.9 we shall see that if $G$ is infinite-dimensional, $D_{l}(G)$ does not coincide with $\overline{\boldsymbol{D}}_{l}(G)$.

Corollary 2. If $D_{1} \in \overline{\boldsymbol{D}}_{l}(G)$ and $D_{2} \in \overline{\boldsymbol{D}}_{r}(G)$, then $D_{1} D_{2}=D_{2} D_{1}$.

Proof. If $D_{1} \in \boldsymbol{D}_{l}(G)$ and $D_{2} \in \boldsymbol{D}_{r}(G)$, we have $D_{1} D_{2}=D_{2} D_{1}$. So the assertion follows from Corollary 1 together with (i) and (ii) of Remark 2.2. q.e.d.

2.7. Support-decreasing continuous linear maps on the spaces $\mathscr{D}(G)$ and $\mathcal{E}(G)$. All discussions in 2.1-2.6, which we made with $\mathscr{D}_{\infty}^{(*)}(G)$ and $\mathcal{E}_{\infty}^{(p)}(G)$ as base spaces, remain valid even if the spaces $\mathscr{D}(G)$ and $\mathcal{E}(G)$ are taken instead. 
The straightforward certification of this fact we leave to the reader. Thus the following in particular hold.

(i) The algebra of all support-decreasing continuous linear maps on $\mathcal{E}(G)$ can be identified, through restriction of maps, with the algebra of all maps on $\mathscr{D}(G)$ having the same properties. This identification preserves the left invariancy of maps.

Let us denote by $\overline{\boldsymbol{D}}_{B}(G)$ these identified algebras.

(ii) For $\bar{D}_{B}(G)$, the topology of uniform convergence on each bounded subset of $\mathcal{E}(G)$ and that of uniform convergence on each bounded subset of $\mathscr{D}(G)$ are equivalent.

We equip $\overline{\boldsymbol{D}}_{B}(G)$ with these equivalent topologies.

(iii) Define the admissible elements of $\mathcal{E}(G)^{I}$ as in the case of $\mathcal{E}_{\infty}(G)^{\tilde{I}}$ (thus, $\left(a_{\nu}\right)_{\nu \in \tilde{I}} \in \mathcal{E}(G)^{\tilde{I}}$ is admissible if and only if it is admissible as element of $\left.\mathcal{E}_{\infty}(G)^{\tilde{I}}\right)$. Then the map $\left(a_{\nu}\right)_{\nu \in \tilde{I}} \mapsto \sum_{\nu \in \tilde{I}} a_{\nu} D_{\nu}$ gives a bijection of all admissible elements of $\mathcal{E}(G)^{I}$ onto $\overline{\boldsymbol{D}}_{B}(G)$, where each $a_{\nu} D_{\nu}$ is viewed as a map on $\mathcal{E}(G)$ or $\mathscr{D}(G)$ (recall Corollary to Theorem 1.4) and $\sum_{\nu \in I} a_{\nu} D_{\nu}$ converges in $\overline{\boldsymbol{D}}_{B}(G)$ unorderedly.

(iv) $\sum_{\nu \in \tilde{I}} a_{\nu} D_{\nu}$ with an admissible element $\left(a_{\nu}\right)_{\nu \in I} \in \mathcal{E}(G)^{\tilde{I}}$ is left invariant if and only if all $a_{\nu}$ are constant.

Let $\left(a_{\nu}\right)_{\nu \in I}$ be an admissible element of $\mathcal{E}(G)^{I}$. Since the topology of $\mathcal{E}(G)$ is finer than $\tau_{p}$ relativized there, it is easy to see that $\sum_{\nu \in I} a_{\nu} D_{\nu}$ as element of $\overline{\boldsymbol{D}}_{B}(G)$ coincides with the restriction to $\mathcal{E}(G)$ of $\sum_{\nu \in I} a_{\nu} D_{\nu}$ as element of $\overline{\boldsymbol{D}}(G)$ in the previous sense. Thus each $D \in \overline{\boldsymbol{D}}_{B}(G)$ is extendable to an element of $\overline{\boldsymbol{D}}(G)$ (needless to say, uniquely). So, apart from topology, $\bar{D}_{B}(G)$ can be viewed as a subalgebra of $\overline{\boldsymbol{D}}(G)$. Under this convention, the subalgebra of $\overline{\boldsymbol{D}}_{B}(G)$ of all left invariant elements coincides with $\overline{\boldsymbol{D}}_{l}(G)$ because of (iv) above and Lemma 2.12. Also, since the map $f \mapsto \check{f}$ on $\mathcal{E}(G)$ is continuous, we see that the automorphism $D \mapsto \check{D}$ of $\overline{\boldsymbol{D}}(G)$ leaves $\overline{\boldsymbol{D}}_{B}(G)$ invariant.

Now let us denote by $\mathcal{E}_{\infty}^{\prime}(G ; e)$ (resp. $\mathcal{E}^{\prime}(G ; e)$ ) the topological subalgebra of $\mathcal{E}_{\infty}^{\prime}(G)$ (resp. $\mathcal{E}^{\prime}(G)$ ) consisting of all elements with support in $\{e\}$ (see (1.11)). Then the left invariant elements of $\overline{\boldsymbol{D}}_{B}(G)$ are in correspondence with the elements of $\mathcal{E}^{\prime}(G ; e)$ (Theorem 1.4). While, each of such elements of $\overline{\boldsymbol{D}}_{B}(G)$ has an extension in $\overline{\boldsymbol{D}}_{l}(G)$ as just mentioned, and each element of $\overline{\boldsymbol{D}}_{l}(G)$ is obtained from some $T \in \mathcal{E}_{\infty}^{\prime}(G ; e)\left(\subseteq \mathcal{E}^{\prime}(G ; e)\right.$ ) (Theorem 1.4). Hence it follows that $\mathcal{E}^{\prime}(G ; e)=\mathcal{E}_{\infty}^{\prime}(G ; e)$ as set.

Remark 2.3. For $C \in \operatorname{Com}(G)$, put $\mathcal{E}^{\prime}(G ; C)=\left\{T \in \mathcal{E}^{\prime}(G) ; \operatorname{supp}(T) \subseteq C\right\}$ and $\mathcal{E}_{\infty}^{\prime}(G ; C)=\left\{T \in \mathcal{E}_{\infty}^{\prime}(G) ; \operatorname{supp}(T) \subseteq C\right\}$. Proposition 1.6, (iii) implies that these two sets are not necessarily identical.

Meantime, as we know ([5]), Proposition 6), $\mathscr{D}^{\prime}(G)$ and $\mathcal{E}^{\prime}(G)$ induce the same topology on each $\mathcal{E}^{\prime}(G ; C)$. It can be similarly shown that $\mathscr{D}_{\infty}^{\prime}(G)$ and $\mathcal{E}_{\infty}^{\prime}(G)$ induce the same topology on each $\mathcal{E}_{\infty}^{\prime}(G ; C)$.

We next demonstrate the following

Theorem 2.4. (i) The map $T \in \mathcal{E}_{\infty}^{\prime}(G ; e) \mapsto D_{T}((1.17))$ is a topological algebra 
isomorphism of $\mathcal{E}_{\infty}^{\prime}(G ; e)$ onto $\overline{\boldsymbol{D}}_{l}(G)$, where $\overline{\boldsymbol{D}}_{l}(G)$ inherits the topology $\mathscr{I}$.

(ii) $\mathcal{E}_{\infty}^{\prime}(G ; e)$ and $\mathcal{E}^{\prime}(G ; e)$ coincide with each other as topological algebra. ${ }^{(5)}$.

As we have already seen, the map $T \in \mathcal{E}_{\infty}^{\prime}(G ; e) \mapsto D_{T} \in \overline{\boldsymbol{D}}_{l}(G)$ is algebraiccally an isomorphism onto (Theorem 1.4) and the sets $\mathcal{E}_{\infty}^{\prime}(G ; e)$ and $\mathcal{E}^{\prime}(G ; e)$ are identical. Now, for any topological linear space $\mathcal{K}$ over $\boldsymbol{C}$, let us denote by $\boldsymbol{B}(\mathcal{K})$ the totality of bounded subsets of $\mathcal{K}$. Then, evidently, one has $\boldsymbol{B}\left(\mathscr{D}_{\infty}^{(*)}(G)\right)$ $\supseteqq \boldsymbol{B}(\mathscr{D}(G))$. For each $\mathscr{B} \in \boldsymbol{B}\left(\mathscr{D}_{\infty}^{(*)}(G)\right)$, define a seminorm $P_{\mathscr{B}}$ on $\overline{\boldsymbol{D}}_{l}(G)$ as $P_{\mathscr{B}}(D)$ $=\sup \{|D f(e)| ; f \in \mathscr{B}\}\left(D \in \overline{\boldsymbol{D}}_{l}(G)\right)$. Let $\mathscr{I}_{1}$ (resp. $\mathscr{I}_{2}$ ) denote the locally convex topology on $\overline{\boldsymbol{D}}_{l}(G)$ determined by $\left\{P_{\mathscr{B}} ; \mathscr{B} \in \boldsymbol{B}\left(\mathscr{D}_{\infty}^{(*)}(G)\right)\right.$ (resp. $\left\{P_{\mathscr{B}} ; \mathscr{B} \in \boldsymbol{B}(\mathscr{D}(G))\right\}$ ), and $\mathscr{I}_{0}$ the relativized $\mathscr{I}$ to $\bar{D}_{l}(G)$. Then we have $\mathscr{I}_{0} \geqq \mathscr{I}_{1} \geqq \mathscr{I}_{2}$. Note that $\mathscr{I}_{1}$ (resp. $\left.\mathscr{I}_{2}\right)$ is nothing but the image of the relativized $\beta\left(\mathscr{D}_{\infty}^{\prime}, \mathscr{D}_{\infty}\right)$ (resp, $\beta\left(\mathscr{D}^{\prime}, \mathscr{D}\right)$ ) to $\mathcal{E}_{\infty}^{\prime}(G ; e)\left(=\mathcal{E}^{\prime}(G ; e)\right)$ under the algebra isomorphism $T \mapsto D_{T}$ of $\mathcal{E}_{\infty}^{\prime}(G ; e)$ onto $\overline{\boldsymbol{D}}_{l}(G)$. Here $\beta\left(\mathscr{D}_{\infty}^{\prime}, \mathscr{D}_{\infty}\right)$ (resp. $\beta\left(\mathscr{D}^{\prime}, \mathscr{D}\right)$ ) coincides with $\beta\left(\mathcal{E}_{\infty}^{\prime}, \mathcal{E}_{\infty}\right)$ (resp. $\beta\left(\mathcal{E}^{\prime}, \mathcal{E}\right)$ ) on $\mathcal{E}_{\infty}^{\prime}(G ; e)\left(=\mathcal{E}^{\prime}(G ; e)\right.$ ) (Remark 2.3). Therefore, to prove the theorem, it suffices to show that $\mathfrak{I}_{0}=\mathscr{I}_{1}=\mathfrak{I}_{2}$ holds.

Lemma 2.13. $I_{0}$ and $I_{1}$ coincide with each other.

Proof. We have only to prove $\mathscr{T}_{1} \geqq \mathscr{I}_{0}$. Let $D^{(i)}$ be any net in $\overline{\boldsymbol{D}}_{l}(G)$ converging to 0 relative to $\mathscr{I}_{1}$. Our task is to show that this net converges to 0 relative to $\mathscr{I}_{0}$. Take any $\mathscr{B} \in \boldsymbol{B}\left(\mathscr{D}_{\infty}^{(*)}(G)\right)$. Then $\mathscr{B}$ is a bounded snbset of $\mathscr{D}_{\infty}^{(r)}(G, N ; C)_{\rho}$ for some $(N, C) \in \boldsymbol{H}\left(G_{1}\right) \times \operatorname{Com}(G), G_{1}$ being a pro-Lie open subgroup of $G$. Put $\mathscr{B}^{\prime}=\left\{{ }_{x} f ; x \in C, f \in \mathscr{B}\right\}$. Then $\mathscr{B}^{\prime}$ is plainly bounded in $\mathscr{D}_{\infty}^{(r)}\left(G, N ; C^{-1} C\right)_{\rho}$ and so $\in \boldsymbol{B}\left(\mathscr{D}_{\infty}^{(*)}(G)\right)$. Since $\overline{\boldsymbol{D}}_{l}(G)$ equipped with $\mathscr{I}_{1}$ is a topological algebra (isomorphic to $\mathcal{E}_{\infty}^{\prime}(G ; e)$ ), the net $D D^{(\lambda)}$ for each $D \in \overline{\boldsymbol{D}}_{l}(G)$ converges to 0 in $\overline{\boldsymbol{D}}_{l}(G)$ relative to $\mathscr{I}_{1}$. Hence $P_{\mathcal{B}^{\prime}}\left(D D^{(\lambda)}\right) \rightarrow 0$, that is, $D\left(D^{(\lambda)} f\right)(x)$ $\left(=D D^{(\lambda)}\left({ }_{x} f\right)(e)\right) \rightarrow 0$ uniformly w.r.t. $f \in \mathscr{B}$ and $x \in C$. Here we see by Lemma 1.17 and Corollary 1 to Proposition 2.3 that $D^{(\lambda)} f \in \mathscr{D}_{\infty}(G, N ; C)_{\rho}(f \in \mathscr{B})$. Therefore, in view of arbitrariness of $D \in \bar{D}_{l}(G), D^{(\lambda)} f \rightarrow 0$ in $\mathscr{D}_{\infty}^{(r)}(G, N ; C)_{\rho}$ (hence in $\mathscr{D}_{\infty}^{(*)}(G)$ ) uniformly w.r.t. $f \in \mathscr{B}$. Since $\mathscr{B}$ is arbitrary, this shows that $D^{(\lambda)}$ $\rightarrow 0$ relative to $T_{0}$. q. e. d.

Lemma 2.14. $I_{1}$ and $I_{2}$ coincide with each other.

Proof. We prove $\mathscr{I}_{2} \geqq \mathscr{I}_{1}$. Let $D^{(\lambda)}$ be any net in $\overline{\boldsymbol{D}}_{l}(G)$ converging to 0 relative to $\mathscr{I}_{2}$ (i.e., $P_{\mathscr{D}}\left(D^{(\lambda)}\right) \rightarrow 0$ for all $\mathscr{B} \in \boldsymbol{B}(\mathscr{D}(G))$ ). Take any $\mathcal{B}_{0} \in \boldsymbol{B}\left(\mathscr{D}_{\infty}^{(*)}(G)\right.$ ). Our task here is to show that $P_{\mathscr{B}_{0}}\left(D^{(\lambda)}\right)$ converges to 0 .

(I) First assume that $G$ is Lie-projective and finite-dimensional. Then there exists a totally disconnected $N \in \boldsymbol{H}_{0}(G)$ and a local homomorphism $\theta$ of $G$ into $N$ such that the map $x \mapsto\left(\pi_{N}^{G}(x), \theta(x)\right)$ is a local isomorphism of $G$ into $(G / N) \times N$ (Lemmas 0.11 and 0.13 ). For each $f \in \mathscr{B}_{0}$ we can choose $h_{f} \in$ $\mathscr{D}_{\infty}((G / N) \times N)$ so that $f(x)=h_{f}\left(\pi_{N}^{G}(x), \theta(x)\right)$ holds for $x$ near $e$ and the set $\left\{h_{f}^{\prime}=h_{f}(\cdot, \theta(e)) ; f \in \mathscr{B}_{0}\right\}$ is bounded in $\mathscr{D}(G / N)$. Put $\mathscr{B}_{0}^{\prime}=\left\{h_{f}^{\prime} \circ \pi_{N}^{G} ; f \in \mathscr{B}_{0}\right\}$.

(5) In $[5] \mathcal{E}^{\prime}(G ; e)$ is denoted by $\boldsymbol{U}(G)$ though, in our notation, $\boldsymbol{U}(G)$ denotes the universal enveloping algebra of $R(G)^{c}$ which corresponds with $D_{l}(G)$. 
Then $\mathscr{B}_{0}^{\prime} \in \boldsymbol{B}(\mathscr{D}(G))$, and $P_{\mathscr{B}_{0}}\left(D^{(\lambda)}\right)=P_{\mathscr{B}_{0}^{\prime}}\left(D^{(\lambda)}\right)$ since $D^{(\lambda)} f(e)=\left(\bar{\pi}_{N}^{G}\left(D^{(\lambda)}\right) h_{f}^{\prime}\right)\left(\pi_{N}^{G}(e)\right)$ $=D^{(\lambda)}\left(h_{f}^{\prime} \circ \pi_{N}^{G}\right)(e)\left(f \in \mathscr{B}_{0}\right)$. Hence $P_{\mathscr{S}_{0}}\left(D^{(\lambda)}\right) \rightarrow 0$.

(II) Next merely assume that $G$ is Lie-projective. Choose $N_{0} \in \boldsymbol{H}(G)$ so that $\mathcal{B}_{0} \subseteq \mathscr{D}_{\infty}\left(G, N_{0}\right)$. For each $\lambda$, choose $D_{0}^{(\lambda)} \in D_{l}(G)$ s.t. $D_{0}^{(\lambda)}=D^{(\lambda)}$ holds on $\mathscr{D}_{\infty}\left(G, N_{0}\right)$ (recall Corollary to Lemma 2.6 and Proposition 2.3). Put $\bar{D}^{(\lambda)}=$ $\bar{\pi}_{N_{0}}^{G}\left(D_{0}^{(\lambda)}\right)\left(\in \boldsymbol{D}_{l}\left(G / N_{0}\right)\right)$. For each $\mathscr{B} \in \boldsymbol{B}\left(\mathscr{D}_{\infty}^{(*)}\left(G, N_{0}\right)\right)$, let $\overline{\mathcal{B}}$ denote the element of $\boldsymbol{B}\left(\mathscr{D}_{\infty}^{(*)}\left(G / N_{0}\right)\right)$ corresponding to $\mathscr{B}$ under the topological linear isomorphism $g \in \mathscr{D}_{\infty}^{(*)}\left(G / N_{0}\right) \mapsto g \circ \pi_{N_{0}}^{G} \in \mathscr{D}_{\infty}^{(*)}\left(G, N_{0}\right)$. As is easily checked, $\boldsymbol{H}_{0}\left(G / N_{0}\right)=\left\{\pi_{N_{0}}^{G}(N)\right.$; $\left.N_{0} \subseteq N \in \boldsymbol{H}_{0}(G)\right\}$. Hence $\boldsymbol{B}\left(\mathscr{D}\left(G / N_{0}\right)\right)=\bigcup\left\{\boldsymbol{B}\left(\mathscr{D}_{\infty}^{(*)}\left(G / N_{0}, \pi_{N_{0}}^{G}(N)\right) ; N_{0} \subseteq N \in \boldsymbol{H}_{0}(G)\right\}\right.$. Here, each $\mathscr{D}_{\infty}^{(*)}\left(G / N_{0}, \pi_{N_{0}}^{G}(N)\right)$ corresponds to $\mathscr{D}_{\infty}^{(*)}(G, N)$ under the above isomorphism. Since $P_{\mathscr{B}}\left(D^{(\lambda)}\right) \rightarrow 0$ for every $\mathscr{B} \in \boldsymbol{B}(\mathscr{D}(G)$ ), it thus follows that $P_{\mathscr{\Phi}}\left(\bar{D}^{(\lambda)}\right) \rightarrow 0$ for every $\overline{\mathscr{A}} \in \boldsymbol{B}\left(\mathscr{D}\left(G / N_{0}\right)\right)$, Since $G / N_{0}$ is Lie-projective and finitedimensional, this implies by (I) that $P_{\mathscr{B}}\left(\bar{D}^{(\lambda)}\right) \rightarrow 0$ for every $\overline{\mathcal{B}} \in \boldsymbol{B}\left(\mathscr{D}_{\infty}^{(*)}\left(G / N_{0}\right)\right)$. Hence, in particular, $P_{\mathscr{B}_{0}}\left(D^{(\lambda)}\right) \rightarrow 0$.

(III) Finally let $G$ be arbitrary. Take a pro-Lie open subgroup $G_{1}$ of $G$. Denote by $\chi$ the indicating function of $G_{1}$. Then $\chi_{\mathscr{B}_{0}} \in \boldsymbol{B}\left(\mathscr{D}_{\infty}^{(*)}\left(G ; G_{1}\right)\right)$ and $P_{\mathscr{B}_{0}}\left(D^{(\lambda)}\right)=P_{\chi \mathcal{B}_{0}}\left(D^{(\lambda)}\right)$. Here we can view $\chi \mathscr{B}_{0}$ as an element of $\boldsymbol{B}\left(\mathscr{D}_{\infty}^{(*)}\left(G_{1}\right)\right)$ and $D^{(\lambda)}$ as a net in $\overline{\boldsymbol{D}}_{l}\left(G_{1}\right)$ s.t. $P_{\mathscr{B}}\left(D^{(\lambda)}\right) \rightarrow 0$ for every $\mathscr{B} \in \boldsymbol{B}\left(\mathscr{D}\left(G_{1}\right)\right)$. Therefore, by (II), $P_{\chi \mathscr{B}_{0}}\left(D^{(\lambda)}\right) \rightarrow 0$. That is, $P_{\mathscr{B}_{0}}\left(D^{(\lambda)}\right) \rightarrow 0$. The proof of the lemma is now complete. q.e.d.

Through the above two lemmas Theorem 2.4 has now been proved.

Finally we touch on the differential operators $u$ on $G$ in Bruhat's sense. They were defined as maps on $\mathscr{D}(G)$ in a somewhat intricate manner in [5], $\mathrm{n}^{\circ} 12$. But, by the sketch given there, it can be been that they are in correspondence with the admissible elements $\left(a_{\nu}\right)_{\nu \in I}$ of $\mathcal{E}(G)^{I}$ in the following way:

$$
u(f)=\sum_{\nu \in I} a_{\nu} T_{D_{\nu}} * f \quad(f \in \mathscr{D}(G))
$$

(for $T_{D_{\nu}}$ see (1.18)). Here the right side is equal to $\sum_{\nu \in I} a_{\nu}\left(\check{f} * \check{T}_{D_{\nu}}\right)^{\nu}=\sum_{\nu \in I} a_{\nu} \check{D}_{\nu} f$. (This reduces to a finite sum for each $f \in \mathscr{D}(G)$ due to Corollary to Lemma 2.6.) Since $\bar{D}_{B}(G)$ is stable under the map $D \mapsto \check{D}$ on $\bar{D}(G)$ as has been mentioned, this after all shows that the differential operators on $G$ in Bruhat's sense are no other than the elements of $\overline{\boldsymbol{D}}_{B}(G)$, i.e., the support-decreasing continuous linear maps on $\mathscr{D}(G)$ (or, what is the same, on $\mathcal{E}(G)$ ).

2.8. The center of the algebra $\bar{D}_{l}(G)$. In this section we describe the center of $\overline{\boldsymbol{D}}_{l}(G)$ by means of the adjoint representation as in the case of Lie groups.

Let $\theta$ be a continuous homomorphism of $G$ into another LC group $G^{\prime}$. Then we have a continuous linear map $\theta_{*}$ of $\mathcal{E}\left(G^{\prime}\right)$ into $\mathcal{E}(G)$ defined by $\theta_{*}(g)=g \circ \theta$ $\left(g \in \mathcal{E}\left(G^{\prime}\right)\right)\left([5]\right.$, Proposition 8). Let $\theta_{*}^{\prime}$ denote the adjoint of $\theta_{*}$ :

$$
\left\langle g, \theta_{*}^{\prime}(T)\right\rangle=\left\langle\theta_{*}(g), T\right\rangle \quad\left(g \in \mathcal{E}\left(G^{\prime}\right), T \in \mathcal{E}^{\prime}(G)\right) .
$$


Since (1.10) holds for $S, T \in \mathcal{E}^{\prime}(G)$ and $\varphi \in \mathcal{E}(G)$, it can be seen that $\theta_{*}^{\prime}(S * T)=$ $\theta_{*}^{\prime}(S) * \theta_{*}^{\prime}(T)\left(S, T \in \mathcal{E}^{\prime}(G)\right)$ holds. Thus $\theta_{*}^{\prime}$ is a continuous homomorphism of $\mathcal{E}^{\prime}(G)$ into $\mathcal{E}^{\prime}\left(G^{\prime}\right)$. Besides, as is easily checked,

$$
\operatorname{supp}\left(\theta_{*}^{\prime}(T)\right) \subseteq \theta(\operatorname{supp}(T)) \quad\left(T \in \mathcal{E}^{\prime}(G)\right) .
$$

Hence in particular $\theta_{*}^{\prime}$ induces a continuous homomorphism of $\mathcal{E}^{\prime}(G ; e)$ into $\mathcal{E}^{\prime}\left(G^{\prime} ; \theta(e)\right)$. Let us denote this homomorphism by $\bar{\theta}$. By Theorem $2.4 \bar{\theta}$ can be viewed also as a continuous homomorphism of $\overline{\boldsymbol{D}}_{l}(G)$ into $\overline{\boldsymbol{D}}_{l}\left(G^{\prime}\right)$. From

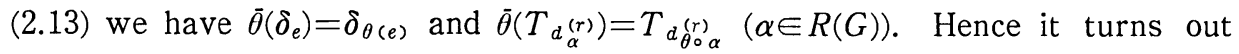
that $\bar{\theta}$ extends the homomorphism of $\boldsymbol{D}_{l}(G)$ into $\boldsymbol{D}_{l}\left(G^{\prime}\right)$ which was introduced at the end of 2.3. Since $\boldsymbol{D}_{l}(G)$ is dense in $\overline{\boldsymbol{D}}_{l}(G)$ (Corollary 1 to Proposition 2.3), this is the unique $I$-continuous extension.

Lemma 2.15. Let $\theta$ be as above. For any $D \in \overline{\boldsymbol{D}}_{l}(G)$ and $g \in \mathcal{E}_{\infty}\left(G^{\prime}\right)$,

$$
(\bar{\theta}(D) g) \circ \theta=D(g \circ \theta) \text {. }
$$

Proof. Evidently, $g \circ \theta\left(g \in \mathcal{E}_{\infty}\left(G^{\prime}\right)\right)$ belongs to $\mathcal{E}_{\infty}(G)$ and $(2.14)$ holds if $D \in$ $\boldsymbol{D}_{l}(G)$. Since $\boldsymbol{D}_{l}(G)$ is dense in $\overline{\boldsymbol{D}}_{l}(G)$ and $\bar{\theta}$ is continuous, the assertion for any $D \in \bar{D}_{l}(G)$ follows at once. q.e.d.

It is obvious that if $\eta \circ \theta$ is a composition of group homomorphisms, then $(\eta \circ \theta)^{-}=\bar{\eta} \circ \bar{\theta}$ holds.

Now, for each $x \in G$, let $I_{G}(x)$ denote the inner automorphism $y \mapsto x y x^{-1}$ of $G$. Then $I_{G}(x)^{-}$is an automorphism of the topological algebra $\overline{\boldsymbol{D}}_{l}(G)$ leaving $\boldsymbol{D}_{l}(G)$ invariant. Since it extends the Lie algebra automorphism $\operatorname{Ad}_{G}(x)$ of $R(G)$ (0.5), let us denote it again by $\operatorname{Ad}_{G}(x)$. Then the $\operatorname{map} \operatorname{Ad}_{G}: x \in G \mapsto \operatorname{Ad}_{G}(x)$ gives a representation of $G$ on $\overline{\boldsymbol{D}}_{l}(G)$ in the algebraic sense. We have obviously $I_{G}(x)_{*}^{\prime}(T)=\delta_{x} * T * \delta_{x^{-1}}\left(x \in G, T \in \mathcal{E}^{\prime}(G)\right)$. Hence, in particular,

$$
\operatorname{Ad}_{G}(x) T=\delta_{x} * T * \delta_{x^{-1}} \quad\left(x \in G, T \in \mathcal{E}^{\prime}(G ; e)\right) .
$$

As is easily checked, we have $D_{\tilde{o}_{x}}=R_{x}(x \in G), R_{x}$ denoting the right translation $f \mapsto f_{x}$ on $\mathcal{E}_{\infty}(G)$. Therefore (2.15) is rewritten as

$$
\operatorname{Ad}_{G}(x) D=R_{x} \circ D \circ R_{x^{-1}} \quad\left(x \in G, D \in \bar{D}_{l}(G)\right) .
$$

Let $\theta$ be a continuous homomorphism of $G$ into $G^{\prime}$ as above. Then $\theta \circ I_{G}(x)$ $=I_{G^{\prime}}(\theta(x)) \circ \theta \quad(x \in G)$. Hence

$$
\bar{\theta} \circ \operatorname{Ad}_{G}(x)=\operatorname{Ad}_{G^{\prime}}(\theta(x)) \circ \bar{\theta} \quad(x \in G) .
$$

Lemma 2.16. Let $j_{0}$ be the canonical injection of $c(G)$ into $G$.

(i) $\bar{j}_{0}$ is a topological algebra isomorphism of $\overline{\boldsymbol{D}}_{l}(c(G))$ onto $\overline{\boldsymbol{D}}_{l}(G)$.

(ii) For each $x \in c(G)$,

holds on $\overline{\boldsymbol{D}}_{l}(c(G))$.

$$
\bar{j}_{0} \circ \operatorname{Ad}_{c(G)}(x)=\operatorname{Ad}_{G}(x) \circ \bar{j}_{0}
$$


Proof. (i): Note that $\bar{j}_{0}$ extends the identity map $d j_{0}: R(c(G)) \mapsto R(G)$ $(=R(c(G)))$ and that the algebra $\boldsymbol{D}_{l}(G)$ is isomorphic to $\boldsymbol{D}_{l}(c(G))$ under $\bar{j}_{0}$, apart from the topology. Then, in view of Remark 2.3 , it is obvious that $\overline{\boldsymbol{D}}_{l}(G)$ is isomorphic to $\overline{\boldsymbol{D}}_{l}(c(G))$ under $\bar{j}_{0}$ including the topology. (ii): This is merely a special case of (2.16). q. e.d.

Lemma 2.17. Suppose $G$ is Lie-projective. Let $D \in \overline{\boldsymbol{D}}_{l}(G)$. If $\bar{\pi}_{N}^{G}(D)=0$ for all $N=\boldsymbol{H}_{0}(G)$, then $D=0$.

Proof. Let $\mathscr{D}(G / N)\left(N \in \boldsymbol{H}_{0}(G)\right)$ be identified with $\mathscr{D}_{\infty}(G, N)$ canonically. If we put $\theta=\pi_{N}^{G}$ in (2.14), it is seen that $\bar{\pi}_{N}^{G}$ behaves as the restriction map $\left.D \in \overline{\boldsymbol{D}}_{l}(G) \mapsto D\right|_{\mathscr{D}_{\infty}(G, N)}$. Hence the assertion. q.e.d.

Theorem 2.5. Let $\overline{\boldsymbol{Z}}(G)$ denote the center of $\overline{\boldsymbol{D}}_{l}(G)$. Then

$$
\overline{\boldsymbol{Z}}(G)=\left\{D \in \overline{\boldsymbol{D}}_{l}(G) ; \operatorname{Ad}_{G}(x) D=D\left(i . e_{.}, R_{x} \circ D=D \circ R_{x}\right) \text { for all } x \in c(G)\right\} .
$$

In particular, $\overline{\boldsymbol{Z}}(G)$ includes $\overline{\boldsymbol{D}}_{l}(G) \cap \overline{\boldsymbol{D}}_{r}(G)$, and coincides with it if $G$ is connected.

Proof. Lemma 2.16 allows us to assume that $G$ is connected. As a special case of (2.16) we have, for each $N \in \boldsymbol{H}_{0}(G), x \in G$ and $D \in \overline{\boldsymbol{D}}_{l}(G)$,

$$
\bar{\pi}_{N}^{G}\left(\operatorname{Ad}_{G}(x) D\right)=\operatorname{Ad}_{G / N}\left(\pi_{N}^{G}(x)\right)\left(\bar{\pi}_{N}^{G}(D)\right) .
$$

Hence, in view of Lemma 2.17, it follows for each $x \in G$ that

$$
\begin{aligned}
& \operatorname{Ad}_{G}(x) D=D \\
& \Longleftrightarrow \operatorname{Ad}_{G / N}\left(\pi_{N}^{G}(x)\right)\left(\bar{\pi}_{N}^{G}(D)\right)=\bar{\pi}_{N}^{G}(D) \text { for all } N \in \boldsymbol{H}_{0}(G) .
\end{aligned}
$$

While, for each $N \in \boldsymbol{H}_{0}(G)$, we have

$$
\begin{aligned}
& \operatorname{Ad}_{G / N}\left(\pi_{N}^{G}(x)\right)\left(\bar{\pi}_{N}^{G}(D)\right)=\bar{\pi}_{N}^{G}(D) \text { for all } x \in G \\
\Longleftrightarrow & \bar{\pi}_{N}^{G}(D) \in \bar{Z}(G / N),
\end{aligned}
$$

since $G / N$ is a connected Lie group. From (2.17) and (2.18),

$$
\begin{gathered}
\operatorname{Ad}_{G}(x) D=D \text { for all } x \in G \\
\Longleftrightarrow \bar{\pi}_{N}^{G}(D) \in \overline{\boldsymbol{Z}}(G / N) \text { for all } N \in \boldsymbol{H}_{0}(G) .
\end{gathered}
$$

Since each $\bar{\pi}_{N}^{G}$ maps $\boldsymbol{D}_{l}(G)$ (hence $\left.\overline{\boldsymbol{D}}_{l}(G)\right)$ onto $\boldsymbol{D}_{l}(G / N)\left(=\overline{\boldsymbol{D}}_{l}(G / N)\right)$, we see again by Lemma 2.17 that the latter condition in (2.19) is equivalent to that $D \in \bar{Z}(G)$, which completes the proof. q.e.d.

Since $\overline{\boldsymbol{D}}_{l}(G)$ is a topological algebra and $\boldsymbol{D}_{l}(G)$ is dense in it, we have the following

Corollary. Let $\boldsymbol{Z}(G)$ denote the center of $\boldsymbol{D}_{l}(G)$. Then 


$$
\begin{aligned}
\boldsymbol{Z}(G) & =\overline{\boldsymbol{Z}}(G) \cap \boldsymbol{D}_{l}(G) \\
& =\left\{D \in \boldsymbol{D}_{l}(G) ; \operatorname{Ad}_{G}(x) D=D \text { for all } x \in c(G)\right\} .
\end{aligned}
$$

The center of $\mathcal{E}^{\prime}(G ; e)$ is given by $\left\{T \in \mathcal{E}^{\prime}(G ; e) ; \delta_{x} * T=T * \delta_{x}\right.$ for all $\left.x \in c(G)\right\}$ (see (2.15)). As against, the center of $\mathcal{E}^{\prime}(G)$ (resp. $\mathcal{E}_{\infty}^{\prime}(G)$ ) is easily found and given by $\left\{T \in \mathcal{E}^{\prime}(G)\right.$ (resp. $\left.\mathcal{E}_{\infty}^{\prime}(G)\right) ; \delta_{x} * T=T * \delta_{x}$ for all $\left.x \in G\right\}$ (use (1.10)).

2.9. Order of differential operators. The order of each $u \in \boldsymbol{U}(G)$ is defined to be the smallest integer $n(\geqq 0)$ s.t. $u \in w\left(\sum_{k=0}^{n} \stackrel{k}{\otimes} R(G)^{c}\right)$, where $w$ denotes the canonical homomorphism of the tensor algebra $\sum_{k=0}^{\infty} \otimes^{k} R(G)^{c}$ onto $\boldsymbol{U}(G)$. We define the order of each $D \in \boldsymbol{D}_{l}(G)$ to be the order of $u \in \boldsymbol{U}(G)$ which corresponds to $D$ (Theorem 2.2). Therefore it equals the smallest integer $n(\geqq 0)$ such that $D$ is expressed as

$$
D=\sum c_{\beta_{1} \cdots \beta_{k}} d_{\beta_{1}}^{(r)} \cdots d_{\beta_{k}}^{(r)} \quad \text { (finite sum), }
$$

where $0 \leqq k \leqq n, \beta_{1}, \cdots, \beta_{k} \in R(G)$ and $c_{\beta_{1} \cdots \beta_{k}} \in C$. For each non-negative integer $n$, let us denote by $\boldsymbol{D}_{l}^{n}(G)$ the linear subspace of $\boldsymbol{D}_{l}(G)$ formed of all the elements of order $\leqq n$.

Remark 2.4. (i) $D_{l}^{n}(G)$ is contained in the $I$-closure of the linear span of $\left\{D_{\nu} ; \nu \in \tilde{I},|\nu| \leqq n\right\}$ in $\boldsymbol{D}_{\iota}(G)$. (Use (i) and (iii) of Remark 2.2.) (ii) If $\left\{\beta_{i}\right\}_{i \in I^{\prime}}$ is an algebraic linear base of $R(G)$, then $\left\{D\left(\Pi_{\nu_{i \neq 0}} \beta_{i}^{\nu i}\right) ;\left(\nu_{i}\right)_{i \in I^{\prime}} \in \tilde{I}^{\prime}, \sum_{i \in I^{\prime}} \nu_{i} \leqq n\right\}$ is an algebraic linear base of $\boldsymbol{D}_{l}^{n}(G)$. Each $D\left(\Pi_{\nu_{i} \neq 0} \beta_{i}^{\nu i}\right)$ has order $\sum_{i \in I^{\prime}} \nu_{i}$.

Definition 2.8. Let $D \in \overline{\boldsymbol{D}}(G)$ and $n$ be a non-negative integer. Take a pro-Lie open subgroup $G_{1}$ of $G$. We say that $D$ satisfies the condition $\left(C_{n}\right)$ if, for each $N \in \boldsymbol{H}_{0}\left(G_{1}\right)$, there can be chosen a finite number of $a_{s} \in \mathcal{E}_{\infty}(G)$ and $D_{s} \in$ $\boldsymbol{D}_{l}^{n}(G)$ so that $D f=\sum_{s} a_{s} D_{s} f$ holds for all $f \in \mathscr{D}_{\infty}(G, N)_{\rho}$. (Because of Lemma 0.16 this condition does not depend on the special choice of $G_{1}$.)

Lemma 2.18. An element $D$ of $D_{l}(G)$ satisfies the condition $\left(C_{n}\right)$ if and only if it has order $\leqq n$.

Proof. Suppose that $D$ satisfies the condition $\left(C_{n}\right)$. For an $N \in \boldsymbol{H}_{0}\left(G_{1}\right)$, let us choose $a_{s} \in \mathcal{E}_{\infty}(G)$ and $D_{s} \in \boldsymbol{D}_{l}^{n}(G)$ as in Definition 2.8. Then $D f(x)=D\left({ }_{x} f\right)(e)$ $=\sum_{s} a_{s}(e) D_{s} f(x)\left(f \in \mathscr{D}_{\infty}(G, N)_{\rho}, x \in G\right)$. In particular, $D=\sum_{s} a_{s}(e) D_{s}$ holds on $\mathscr{D}_{\infty}\left(G, N ; G_{1}\right)_{\rho}\left(\cong \mathscr{D}_{\infty}\left(G_{1}, N\right)\right)$. Each element of $\boldsymbol{D}_{l}(G)$ can be viewed as an element of $\boldsymbol{D}_{l}\left(G_{1}\right)$. Therefore, from the last equality, we have $\bar{\pi}_{N}^{G}(D)=$ $\sum_{s} a_{s}(e) \bar{\pi}_{N}^{G}\left(D_{s}\right)$. In view of (ii) of Remark 2.4 and Lemma 2.5, it is not difficult to see that $\bar{\pi}_{N^{1}}^{C_{1}(D)}$ has the same order as $D$ if $N$ is taken to be sufficiently small. On the other hand, whatever $N$ may be, $\sum_{s} a_{s}(e) \bar{\pi}_{N^{1}}^{G_{1}}\left(D_{s}\right)$ has order $\leqq n$ as well as each $D_{s}$. Hence it follows that $D$ has order $\leqq n$. This proves the "only if" part of the lemma. The "if" part is trivial. q.e.d. 
We now make the following definition, the consistency of which with the orders of the elements of $\boldsymbol{D}_{\iota}(G)$ previously mentioned is assured by Lemma 2.18 .

Definition 2.9. Suppose that an element $D$ of $\overline{\boldsymbol{D}}(G)$ satisfies the condition $\left(C_{n}\right)$ for some $n$. In this case we say that $D$ has order $\leqq n$, and call the minimum of such $n$ 's the order of $D$. If $D$ satisfies the condition $\left(C_{n}\right)$ for no $n$, we say that $D$ has infinite order.

Proposition 2.4. Let $D=\sum_{\nu \in I} a_{\nu} D_{\nu}$, where $\left(a_{\nu}\right)_{\nu \in I}$ is an admissible element of $\mathcal{E}_{\infty}(G)^{I}$, and $n$ be a non-negative integer. For $D$ to have order $\leqq n$ (i.e., to satisfy the condition $\left.\left(C_{n}\right)\right)$, it is necessary and sufficient that $a_{\nu}=0$ unless $|\nu| \leqq n$.

Proof. The sufficiency of the condition is obvious from Corollary to Lemma 2.6. We now prove its necessity. So assume that $D$ has order $\leqq n$. For any $\nu^{(0)} \in \tilde{I}$ with $\left|\nu^{(0)}\right|>n$ and $x_{0} \in G$, choose $f \in \mathscr{D}(G)$ s.t. $D_{\nu} f\left(x_{0}\right)=1$ if $\nu=\nu^{(0)}$, and $=0$ otherwise (Corollary to Lemma 2.10). Since $f$ belongs to $\mathscr{D}_{\infty}(G, N)_{\rho}$ for some $N \in \boldsymbol{H}_{0}\left(G_{1}\right), G_{1}$ being a pro-Lie open subgroup of $G$, our assumption enables us to have a finite number of $a_{s} \in \mathcal{E}_{\infty}(G)$ and $D_{s} \in \boldsymbol{D}_{l}^{n}(G)$ s.t. $D f=\sum_{s} a_{s} D_{s} f$. Here, each $D_{s}$ belongs to the $\mathcal{I}$-closure of the linear span of $\left\{D_{\nu} ; \nu \in \tilde{I},|\nu| \leqq n\right\}$ (Remark 2.4, (i)). Hence, by the choice of $f, D_{s} f\left(x_{0}\right)=0\left({ }^{\forall} s\right)$, and so, $D f\left(x_{0}\right)=0$. Therefore, again by the choice of $f, a_{\nu}(0)\left(x_{0}\right)=0$. Since $\nu^{(0)}$ and $x_{0}$ are arbitrary, the proof is complete. q.e.d.

As an easy consequence of Proposition 2.4 together with Corollary to Lemma 2.10 we have the following

Corollary 1. Let $n$ be a non-negative integer. All elements of $\overline{\boldsymbol{D}}(G)$ of order $\leqq n$ form a closed linear subspace of $\overline{\mathbf{D}}(G)$. $\overline{\boldsymbol{D}}(G)$.

Corollary 2. All elements of $\overline{\boldsymbol{D}}(G)$ of finite order constitute a subalgebra of

Proof. Suppose that $D_{j} \in \overline{\boldsymbol{D}}(G)(j=1,2)$ has order $\leqq n_{j}$. To prove the assertion, it suffices to show that $D_{1} D_{2}$ has order $\leqq n_{1}+n_{2}$. By assumption $D_{j}$ is expressed as $D_{j}=\sum_{\nu \in I} a_{\nu}^{(j)} D_{\nu}$ with an admissible element $\left(a_{\nu}^{(j)}\right)_{\nu \in I}$ of $\mathcal{E}_{\infty}(G)^{I}$ s.t. $a_{\nu}^{(j)}=0$ unless $|\nu| \leqq n_{j}$. It is evident that each $a_{\nu}^{(1)} D_{\nu} \circ a_{\nu^{\prime}}^{(2)}(\in \bar{D}(G))$ satisfies the condition $\left(C_{|\nu|}\right)$ and so has order $\leqq|\nu|$. Hence, by Corollary $1, D_{1^{\circ}} \circ a_{\nu^{\prime}}^{(2)}$ $\left(=\sum_{\nu \in I}\left(a_{\nu}^{(1)} D_{\nu^{\circ}} a_{\nu^{\prime}}^{(2)}\right)\right)$ has order $\leqq n_{1}$. Since $D_{\nu^{\prime}}$ leaves each $\mathscr{D}_{\infty}(G, N)_{\rho}\left(N \in \boldsymbol{H}_{0}\left(G_{1}\right)\right.$, $G_{1}$ a pro-Lie open subgroup of $G$ ) invariant (Lemma 1.17), it then follows that $D_{1} \circ a_{\nu^{\prime}}^{(2)} D_{\nu^{\prime}}$ has order $\leqq n_{1}+\left|\nu^{\prime}\right|$. Hence, again by Corollary $1, D_{1} D_{2}$ has order $\leqq n_{1}+n_{2}$. q. e. d.

Corollary 3. Let $D \in \bar{D}(G) . \quad D$ and $\check{D}$ have the same order (finite or infinite).

Proof. It suffices to show, for each integer $n \geqq 0$, that if $D$ has order $\leqq n$, then so does $\check{D}$. Take the admissible element $\left(a_{\nu}\right)_{\nu \in I}$ of $\mathcal{E}_{\infty}(G)^{I}$ s. t. $D=\sum_{\nu \in I} a_{\nu} D_{\nu}$. 
Then $\check{D}=\sum_{\nu \in I} \check{a}_{\nu} \check{D}_{\nu}$. Here, by assumption, $a_{\nu}=0$ unless $|\nu| \leqq n$. Meanwhile, each $d_{\alpha}^{(l)}(\alpha \in R(G))$ has order $\leqq 1$ because it is a derivation (recall Lemma 2.11). Therefore, as we see from the proof of Corollary 2 , each $\check{\alpha}_{\nu} \check{D}_{\nu}$ has order $\leqq|\nu|$. Hence, by Corollary $1, \check{D}$ has order $\leqq n$. q. e. d.

Corollary 4. For each non-negative integer $n$, let $\overline{\boldsymbol{D}}_{l}^{n}(G)$ denote the linear subspace of $\overline{\boldsymbol{D}}_{l}(G)$ formed of all elements of order $\leqq n$. Then $\overline{\boldsymbol{D}}_{l}^{n}(G)$ inheriting $I$ is a minimal locally convex space with the topological linear base $\left\{D_{\nu} ; \nu \in \tilde{I}\right.$, $|\nu| \leqq n\}$.

Proof. Put $\tilde{I}_{n}=\{\nu \in \tilde{I} ;|\nu| \leqq n\}$ and consider the minimal locally convex

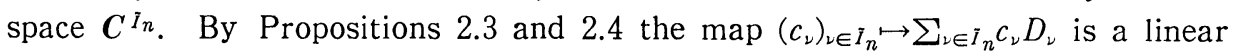
bijection of $C^{\tilde{I}_{n}}$ onto $\overline{\boldsymbol{D}}_{l}^{n}(G)$. In view of Proposition 1.1, Lemma 1.17 and Corollary to Lemma 2.6 we see easily that this bijection is topological. q.e.d.

As promised in 2.6, let us see here that if $G$ is infinite-dimensional, then $\boldsymbol{D}_{l}(G) \neq \overline{\boldsymbol{D}}_{l}(G)$. Indeed, we can take in this case an infinite sequence $\gamma_{i_{k}}(k=$ $1,2,3, \cdots)$ of mutually distinct elements of the base $\left\{\gamma_{i}\right\}_{i \in I}$. Then the element $\sum_{k=1}^{\infty}\left(d_{r_{i_{k}}}^{(r)}\right)^{k}$ of $\overline{\boldsymbol{D}}_{l}(G)$ (Proposition 2.3), for instance, has infinite order (Proposition 2.4) and so does not belong to $\boldsymbol{D}_{l}(G)$.

Proposition 2.5. Let $n$ be a positive integer. Each $D \in \overline{\boldsymbol{D}}(G)$ of order $\leqq n$ can be extended uniquely to a continuous linear map of $\mathcal{E}_{n}^{(p)}(G)$ into $\mathcal{E}_{0}(G)$. This extension decreases the supports of all $f \in \mathcal{E}_{n}(G)$.

Proof. Since $\mathcal{E}_{\infty}(G)$ is dense in $\mathcal{E}_{n}^{(p)}(G)$, the uniqueness is obvious. Let us see that the extension is possible. By assumption $D$ is expressed as $D=$ $\sum_{\nu \in \tilde{I}_{n}} a_{\nu} D_{\nu}\left(a_{\nu} \in \mathcal{E}_{\infty}(G)\right)$, where $\tilde{I}_{n}=\{\nu \in \tilde{I} ;|\nu| \leqq n\}$. It is evident that each $D_{\nu}$ s.t. $|\nu| \leqq n$ can act as a continuous linear map of $\mathscr{D}_{\infty}^{(*)}(G)$ into $\mathcal{E}_{0}(G)$. Now consider the formal sum $\sum_{\nu \in I_{n}} a_{\nu} D_{\nu} f\left(f \in \mathscr{D}_{n}(G)\right)$. Take a pro-Lie open subgroup $G_{1}$ of $G$. From Lemmas 1.17 and 2.6 it can be seen that this sum reduces to a finite sum on each $\mathscr{D}_{n}(G, N)_{\rho}\left(N \in \boldsymbol{H}\left(G_{1}\right)\right)$. Therefore it makes sense and we can define a continuous linear map $\tilde{D}$ of $\mathscr{D}_{n}^{(*)}(G)$ into $\mathcal{E}_{0}(G)$ by $\tilde{D} f=$ $\sum_{\nu \in \tilde{I}_{n}} a_{\nu} D_{\nu} f\left(f \in \mathscr{D}_{n}(G)\right)$. For a $C \in \operatorname{Com}(G)$, take $a \in \mathscr{D}(G)$ s.t. $a(X) \equiv 1$ on a neighbourhood of $C$. Then, since $\tilde{D}$ decreases supports, we have $P_{C}(\tilde{D} \varphi)=$ $P_{C}(\tilde{D}(a \varphi))$ for $\varphi \in \mathscr{D}_{n}(G)$. Hence we see that $\tilde{D}$ is continuous also as a map of $\mathscr{D}_{n}^{(p)}(G)$ into $\mathcal{E}_{0}(G)$. Since $\mathscr{D}_{n}(G)$ is dense in $\mathcal{E}_{n}^{(p)}(G)$ and $\mathcal{E}_{0}(G)$ is complete, this shows that $\tilde{D}$ extends to a continuous linear map of $\mathcal{E}_{n}^{(p)}(G)$ into $\mathcal{E}_{0}(G)$. By construction of $\tilde{D}$, this extension coincides with $D$ on $\mathscr{D}_{\infty}(G)$, hence on $\mathcal{E}_{\infty}(G)$. Furthermore, since it decreases the support of each $f \in \mathscr{D}_{n}(G)$, we can show in parallel to the proof of Lemma 2.3, (ii) that it decreases the supports of all $f \in \mathcal{E}_{n}(G)$. q. e. d.

Note. Let $\boldsymbol{D}(G)$ denote the subalgebra of $\overline{\boldsymbol{D}}(G)$ generated by $\boldsymbol{D}_{l}(G) \cup \boldsymbol{D}_{r}(G)$ $\cup \mathcal{E}_{\infty}(G)$ algebraically. In [6], where $G$ is compact, we defined the differential 
operators on $G$ as the elements of $\boldsymbol{D}(G)$. For our purpose there to discuss $\boldsymbol{U}(G)$, such a definition was enough. But $\boldsymbol{D}(G)$ does not in general coincide with $\overline{\boldsymbol{D}}(G)$. In fact, $\boldsymbol{D}(G)$ contains no element of infinite order (Corollaries 2 and 3 to Proposition 2.4). If $G$ is finite-dimensional, $\boldsymbol{D}(G)$ coincides with the set of all elements of $\overline{\boldsymbol{D}}(G)$ with finite order. In general, however, this is false. For instance, in case $G$ is abelian and infinite-dimensional, it can be seen without difficulty that $\boldsymbol{\delta}(G) \backslash \boldsymbol{D}(G) \neq \varnothing$. For $\boldsymbol{D}(G)$ to coincide with $\overline{\boldsymbol{D}}(G)$, it is necessary and sufficient that one of the following two conditions is fulfilled: (a) $G$ is totally disconnected; or (b) $G$ is compact and finite-dimensional.

2.10. On derivable distributions in Bruhat's sense. Let $n$ be a nonnegative integer. In [5], $\mathrm{n}^{\circ} 12$ a distribution $T \in \mathscr{D}^{\prime}(G)$ was said to be $n$-times derivable if $T_{D^{*}} T$ is a continuous function on $G$ for every $D \in \overline{\boldsymbol{D}}_{l}^{n}(G)$. $^{(6)}$ But, if $G$ is not locally connected, the substance of such a $T \in \mathscr{D}^{\prime}(G)$ has been left unknown. We now show that such a $T$ is no other than a function in $\mathcal{E}_{n}(G)$ no matter $G$ is locally connected or not.

Lemma 2.19. Let $n$ be a positive integer. For each $f \in \mathcal{E}_{n}(G)$ and $D \in \overline{\boldsymbol{D}}_{l}^{n}(G)$, there holds

$$
T_{D^{*}} f=\check{D} f \quad \text { (as distribution), }
$$

where $\check{D}$ is viewed as a continuous linear map of $\mathcal{E}_{n}^{(p)}(G)$ into $\mathcal{E}_{0}(G)$ (recall Proposition 2.5 and Corollary 3 to Proposition 2.4).

Proof. It is evident that the map $f \in \mathcal{E}_{0}(G) \rightarrow f \in \mathscr{D}^{\prime}(G)$ is continuous. Hence so is the map $f \in \mathcal{E}_{n}^{(p)}(G) \mapsto \check{f} \in \mathscr{D}^{\prime}(G)$. Since $\left\langle\varphi, \check{f} * \check{T}_{D}\right\rangle=\left\langle\varphi * T_{D}, \check{f}\right\rangle((1.15))$ and $\varphi * T_{D} \in \mathscr{D}(G)$ (Lemma 1.24 and (1.13)) for $\varphi \in \mathscr{D}(G)$ and $f \in \mathcal{E}_{n}(G)$, it then follows that the map $f \in \mathcal{E}_{n}^{(p)}(G) \mapsto T_{D^{*}} f\left(=\left(\check{f} * \check{T}_{D}\right)^{`}\right) \in \mathscr{D}^{\prime}(G)$ is continuous (recall that the map $T \mapsto \check{T}$ is continuous). Since $\mathscr{D}(G)$ is dense in $\mathcal{E}_{n}^{(p)}(G)$ and $T_{D^{*}} f=\check{D} f$ holds for $f \in \mathscr{D}(G)$, it is now clear that $T_{D^{*}} f=\check{D} f$ holds for $f \in \mathcal{E}_{n}(G)$. q. e.d.

Lemma 2.20. Let $f \in \mathcal{E}_{0}(G)$. If $T_{d_{\alpha}^{(r)} * f}$ belongs to $\mathcal{E}_{0}(G)$ for every $\alpha \in$ $R(G)$, then $f \in \mathcal{E}_{1}(G)$.

Proof. For each $\alpha \in R(G)$, put $f_{\alpha}=T_{d_{\alpha}^{(r)} * f}\left(\in \mathcal{E}_{0}(G)\right)$. Then, for $\varphi \in \mathscr{D}(G)$,

$$
\begin{aligned}
& \int_{G} \varphi(x) f_{\alpha}(\alpha(-t) x) d_{G} x \\
= & \int_{G} \varphi(\alpha(t) x) f_{\alpha}(x) d_{G} x=\left\langle\alpha(t) \varphi, T_{d_{\alpha}^{(r) * f}}\right\rangle \\
= & \left\langle\int_{G} \varphi(\alpha(t) y x) f(x) d_{G} x,\left(T_{\left.d_{\alpha}^{(r)}\right)}\right\rangle\right.
\end{aligned}
$$

(6) By Theorem 2.4 and Corollary 4 to Proposition 2.4 it is seen that $\left\{T_{D} ; D \in \overline{\boldsymbol{\nu}}_{l}^{n}(G)\right\}$ is identical with $\boldsymbol{U}^{n}(G)$ appearing in [5]. 


$$
\begin{aligned}
& =\frac{d}{d t} \int_{G} \varphi(\alpha(t) x) f(x) d_{G} x \\
& =\frac{d}{d t} \int_{G} \varphi(x) f(\alpha(-t) x) d_{G} x .
\end{aligned}
$$

Hence

$$
\int_{G} \varphi(x) d_{G} x \int_{0}^{s} f_{\alpha}(\alpha(-t) x) d t=\int_{G} \varphi(x)\{f(\alpha(-s) x)-f(x)\} d_{G} x .
$$

Since $\varphi$ is arbitrary, this implies that

$$
f(\alpha(-s) x)-f(x)=\int_{0}^{s} f_{\alpha}(\alpha(-t) x) d t \quad(x \in G) .
$$

Thus $d_{\alpha}^{(l)} f$ exists and equals $f_{\alpha}$. Since $\alpha$ is arbitrary, this shows that $f \in \mathcal{E}_{1}(G)$. q.e.d.

Lemma 2.21. Let $n$ be a non-negative integer and $T \in \mathscr{D}^{\prime}(G)$. If $T_{D\left(\alpha_{p}, \cdots, \alpha_{1}\right)} * T$ belongs to $\mathcal{E}_{0}(G)$ for all $D\left(\alpha_{p}, \cdots, \alpha_{1}\right)=d_{\alpha_{p}}^{(r)} \cdots d_{\alpha_{1}}^{(r)}\left(0 \leqq \underline{p} \leqq n, \alpha_{1}, \cdots, \alpha_{p} \in R(G)\right)$, then $T \in \mathcal{E}_{n}(G)$.

Proof. Since $T_{1}=\delta_{e}$, the assumption of the lemma implies in particular that $T \in \mathcal{E}_{0}(G)$. So let us write $f$ for $T$. We now show by finite induction that $f$ belongs to $\mathcal{E}_{k}(G)$ for $k=1, \cdots, n$. To do so, assume for a $k<n$ that $f \in \mathcal{E}_{k}(G)$. Then, by Lemma 2.19 and the assumption of the present lemma,

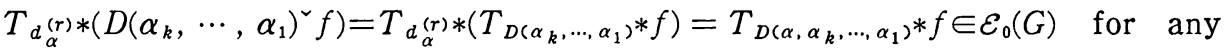
$\alpha_{1}, \cdots, \alpha_{k}, \alpha \in R(G)$. Since $D\left(\alpha_{k}, \cdots, \alpha_{1}\right)^{2} f \in \mathcal{E}_{0}(G)$, this shows by Lemma 2.20 that $D\left(\alpha_{k}, \cdots, \alpha_{1}\right)^{2} f \in \mathcal{E}_{1}(G)$. Hence $f \in \mathcal{E}_{k+1}(G)$, which completes the induction. q. e. d.

Lemmas 2.19 and 2.21 establish the following

Theorem 2.6. Let $n$ be a non-negative integer and $T \in \mathscr{D}^{\prime}(G) . \quad T_{D^{*}} T$ belongs to $\mathcal{E}_{0}(G)$ for every $D \in \overline{\boldsymbol{D}}_{l}(G)$ of order $\leqq n$ if and only if $T$ is a function in $\mathcal{E}_{n}(G)$.

\section{Department of Mathematics \\ College of ScIENCE AND TechNology \\ NIHON UNIVERSITY}

\section{References}

[1] H. Boseck and G. Czichowski, Grundfunktionen und veralgemeinerte Funktionen auf topologischen Gruppen I, II, Math. Nachr., 58 (1973), 215-240, 66 (1975), 319-332.

[2] H. Boseck and G. Czichowski, Connectedness properties of locally compact groups, Math. Nachr., 89 (1979), 17-24.

[3] H. Boseck, G. Czichowski and K.P. Rudolph, Analysis on topological groupsgeneral Lie theory, Teubner, Leipzig, 1981.

[4] N. Bourbaki, Éléments de mathématique, Espaces vectoriels topologiques, Hermann, Paris, 1954. 
[5] F. Bruhat, Distributions sur un groupe localement compact et applications à l'étude des représentations des groupes p-adiques, Bull. Soc. Math. France, 89 (1961), 43-75.

[6] T. Edamatsu, Spaces of differentiable functions on compact groups, J. Math. Kyoto Kyoto Univ., 24 (1984), 323-360.

[7] S. Helgason, Differential operators on homogeneous spaces, Acta Math., 102 (1959), 239-299.

[8] K. Iwasawa, On some types of topological groups, Ann of Math., 50 (1949), 507-558.

[9] R.K. Lashof, Lie algebras of locally compact groups, Pacific J. Math., 7 (1957), 1145-1162.

[10] D. Montgomery and L. Zippin, Topological transformation groups, Interscience, New York, 1955.

[11] L.S. Pontryagin, Topological groups (Russian), Gostehizdat, Moscow, 1954.

[12] J. Riss, Éléments de calcul différentiel et théorie des distributions sur les groupes abéliens localement compacts, Acta Math., 89 (1953), 45-105. 